Florida International University FIU Digital Commons

$11-5-2014$

\title{
Characterization of Juvenile Hormone Biosynthetic Enzymes in the Mosquito, Aedes aegypti
}

Pratik Nyati

Florida International University, pnyat001@fiu.edu

DOI: $10.25148 /$ etd.FI14110768

Follow this and additional works at: https:// digitalcommons.fiu.edu/etd

Part of the Biochemistry, Biophysics, and Structural Biology Commons, Biology Commons, and the Entomology Commons

\section{Recommended Citation}

Nyati, Pratik, "Characterization of Juvenile Hormone Biosynthetic Enzymes in the Mosquito, Aedes aegypti" (2014). FIU Electronic Theses and Dissertations. 1688.

https://digitalcommons.fiu.edu/etd/1688 


\section{FLORIDA INTERNATIONAL UNIVERSITY}

Miami, Florida

\section{CHARACTERIZATION OF JUVENILE HORMONE BIOSYNTHETIC ENZYMES IN}

THE MOSQUITO, AEDES AEGYPTI

A dissertation submitted in partial fulfillment of the

requirements for the degree of

DOCTOR OF PHILOSOPHY

in

BIOLOGY

by

Pratik Nyati

2014 
To: Interim Dean Michael R. Heithaus

College of Arts and Sciences

This dissertation, written by Pratik Nyati, and entitled Characterization of Juvenile Hormone Biosynthetic Enzymes in the Mosquito, Aedes aegypti, having been approved in respect to style and intellectual content, is referred to you for judgment.

We have read this dissertation and recommend that it be approved.

Alejandro Barbieri

$\begin{array}{r}\hline \text { Lidia Kos } \\ \hline \text { Lou W. Kim } \\ \hline \text { Kalai Mathee } \\ \hline \text { Fernando G. Noriega, Major Professor }\end{array}$

Date of Defense: November 5, 2014

This dissertation of Pratik Nyati is approved.

Interim Dean Michael R. Heithaus

College of Arts and Science

Dean Lakshmi N. Reddi

University Graduate School

Florida International University, 2014 


\section{DEDICATION}

This dissertation is dedicated to my parents, Dr. Prem Nyati and Brijlata Nyati for instilling in me the importance of education, hard work and a keen desire to run after the new learning. This doctoral thesis is also dedicated to my lovely better half Parul and younger brother Prakhar for a constant support and encouragement at each moment of my life. 


\section{ACKNOWLEDGMENTS}

Firstly I would like to thank the almighty, from deep core of my heart, for showering blessings and success throughout my life.

Words are not sufficient to show the gratitude to my mentor Dr. Fernando G. Noriega for providing me a constant support and liberty to pursue this project. His exceptional guidance, advice and patience allowed me to successfully accomplish this goal of life. I am fortunate enough to have him as a mentor and as a great advisor; he has been a great friend during the entire program. The credit of this entire learning goes to him. Thanks a lot Dr. Noriega for lightning the path of my success as a wonderful mentor.

I thank all the members of Noriega laboratory, both present and past, for their support. I would like to give the very special thanks to Dr. Marcela Nouzova and Dr. Crisalejandra Rivera Perez for their constant guidance and assistance with my project. Working in the lab was so easy, pleasant and unforgettable because of them. I would also like to thank Dr. Jaime Mayoral, Dr. Mark Clifton and Dr. Mario Perez for their help in my research.

I would like to thank my committee members, Dr. Alejandro Barbieri, Dr. Kalai Mathee, Dr. Lidia Kos and Dr. Lou W. Kim for their helpful ideas, guidance, and critical reading of my dissertation. I thank Department of Biology, FIU for the financial support as a Teaching Assistant throughout this dissertation.

A friend in need is a friend indeed; it has been proved by my friends Dr. Vidya Sagar, Jitesh Pillai, Gorakh Tatke and many others. I thank all of them for all our 
discussions on technical, non-technical and all the world issues and making my stay such a pleasant experience.

Also the credit of a great belief on my capability goes to my parent in-law, Maheshji and Sadhnaji and the very lovely and dashing brother in law Pulkit Maheshwari.

I am wordless to thank my dearest mummy -papa, Lata \& Dr. Prem Nyati, and the great enthusiastic and devoted brother Prakhar, for being always there with a full hearted support and encouragement to enlighten my path.

None of this would have been possible without my better half, Parul Maheshwari. Thank you dear Parul for your unconditional love, belief and sharing all the moments of happiness and sadness together. 


\title{
ABSTRACT OF THE DISSERTATION \\ CHARACTERIZATION OF JUVENILE HORMONE BIOSYNTHETIC ENZYMES IN THE MOSQUITO AEDES AEGYPTI
}

\author{
by \\ Pratik Nyati \\ Florida International University, 2014 \\ Miami, Florida

\section{Professor Fernando G. Noriega, Major Professor}

The juvenile hormones (JHs) are sesquiterpenoid compounds that play a central role in insect reproduction, development and behavior. They are synthesized and secreted by a pair of small endocrine glands, the corpora allata (CA), which are intimately connected to the brain. The enzymes involved in the biosynthesis of $\mathrm{JH}$ are attractive targets for the control of mosquito populations. This dissertation is a comprehensive functional study of five Aedes aegypti CA enzymes, HMG-CoA synthase (AaHMGS), mevalonate kinase $(A a \mathrm{MK})$, phosphomevalonate kinase $(A a \mathrm{PMK})$, farnesyl diphosphate synthase (AaFPPS) and farnesyl pyrophosphate phosphatase (AaFPPase).

The enzyme AaHMGS catalyzes the condensation of acetoacetyl-CoA and acetylCoA to produce HMG-CoA. The enzyme does not require any co-factor, although its activity is enhanced by addition of $\mathrm{Mg}^{2+}$. The enzyme $A a \mathrm{MK}$ is a class I mevalonate kinase that catalyzes the ATP-dependent phosphorylation of mevalonic acid to form mevalonate 5-phosphate. Activity of $A a \mathrm{MK}$ is inhibited by isoprenoids. The enzyme AaPMK catalyzes the cation-dependent reversible reaction of phosphomevalonate and ATP to form diphosphate mevalonate and ADP. The enzyme AaFPPS catalyzes the 
condensation of isopentenyl diphosphate (IPP) and dimethylallyl pyrophosphate (DMAPP) to form geranyl diphosphate (GPP) and farnesyl pyrophosphate (FPP). The enzyme AaFPPS shows an unusual product regulation mechanism, with chain length final product of 10 or $15 \mathrm{C}$ depending on the metal cofactor present. The enzymes AaFPPase-1 and AaFPPase-2 efficiently hydrolyze FPP into farnesol, although RNAi experiments demonstrate that only AaFPPase-1 is involved in the catalysis of FPP into FOL in the CA of $A$. aegypti. This dissertation also explored the inhibition of the activity of some of the JH biosynthesis enzymes as tools for insect control. We described the effect of N-acetylS-geranylgeranyl-L-cysteine as a potent inhibitor of AaFPPase 1 and AaFPPase-2. In addition, inhibitors of $A a \mathrm{MK}$ and AaHMGS were also investigated using purified recombinant proteins.

The present study provides an important contribution to the characterization of recombinant proteins, the analysis of enzyme kinetics and inhibition constants, as well as the understanding of the importance of these five enzymes in the control of $\mathrm{JH}$ biosynthesis rates. 


\section{TABLE OF CONTENTS}

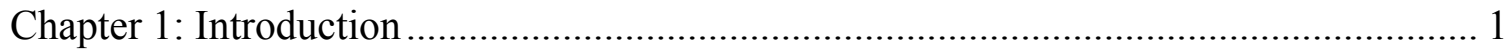

1.1 Aedes aegypti as the principal vector of dengue, yellow fever and chickungunya .. 1

1.2 Brief history on JH research and the discovery of JH structures. ............................. 3

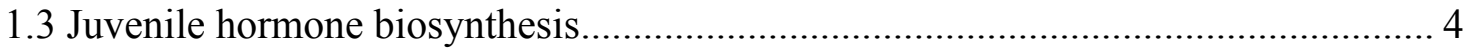

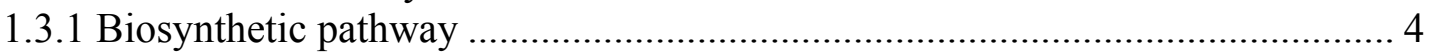

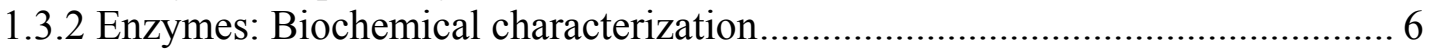

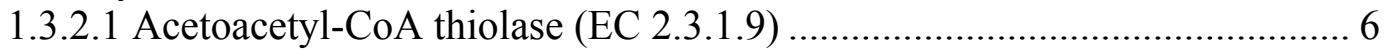

1.3.2.2 HMG-CoA synthase (HMGS) (EC 2.3.3.10)........................................... 6

1.3.2.3 HMG-CoA reductase (HMGR) (EC 1.1.1.34) ......................................... 6

1.3.2.4 Mevalonate kinase (MK) (EC 2.7.1.36).................................................. 7

1.3.2.5 Phosphomevalonate kinase (PMK) (EC 2.7.4.2) ...................................... 7

1.3.2.6 Mevalonate diphosphate decarboxylase (MDD) (EC 4.1.1.33)................. 7

1.3.2.7 Isopentenyl diphosphate isomerase (IPPI) (EC 5.3.3.2) ………................ 8

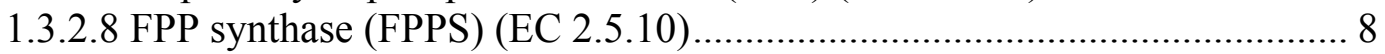

1.3.2.9 FPP phosphatase (FPPase) (EC 3.1.7.6) ………..................................... 9

1.3.2.10 Farnesol dehydrogenase (FOLD) ......................................................... 9

1.3.2.11 Farnesal dehydrogenase (FALD) (EC 1.2.1.B9) ................................... 9

1.3.2.12 JH acid methyltransferase (JHAMT) (EC 2.1.1.15) ............................... 10

1.3.2.13 MF epoxidase (Epox) (3.6.2.4) ………............................................. 10

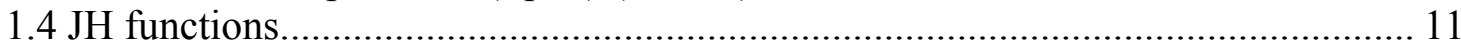

1.4.1 JH is one of the key hormones regulating growth and metamorphosis of

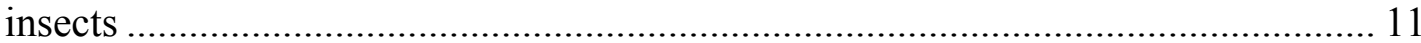

1.4.2 JH assesses nutritional information to regulate reproduction in mosquitoes ... 11

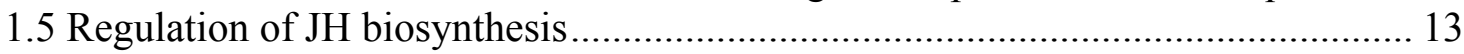

1.5.1 Role of the insulin/TOR signaling pathway …………………......................... 13

1.5.2 JH biosynthesis regulation by allatotropins and allatostatins ........................... 15

1.5.3 Regulation of JH synthesis by 20-hydroxyecdysone and ecdysis triggering

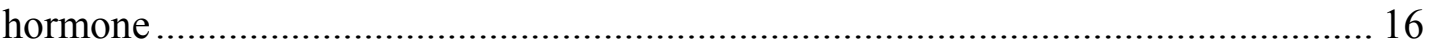

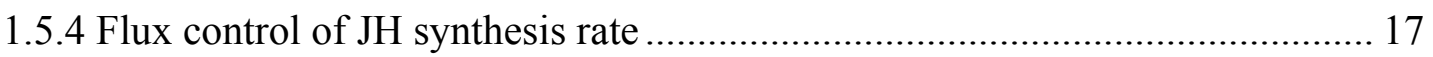

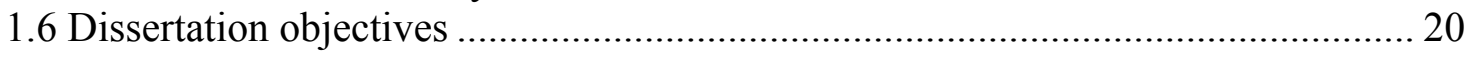

1.7 Dissertation organization............................................................................ 21

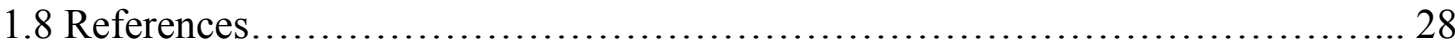

Chapter 2: Farnesyl phosphatase, a corpora allata enzyme involved in juvenile

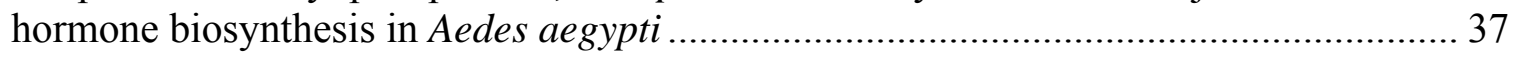

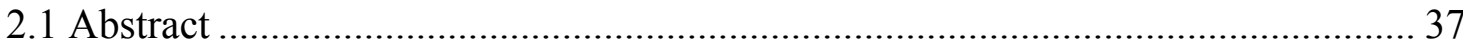

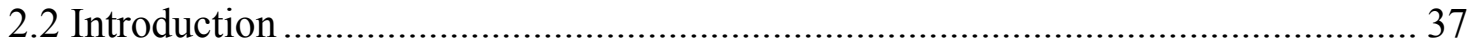

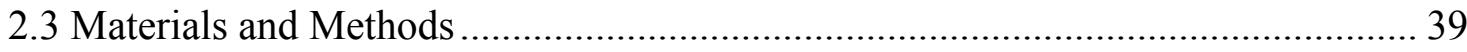

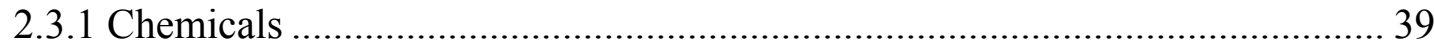

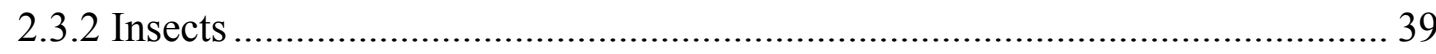

2.3.3 Expression of recombinant AaFPPases ........................................................ 39 


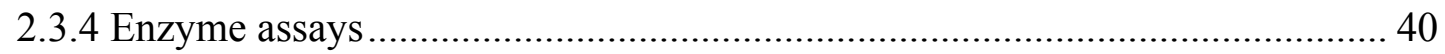

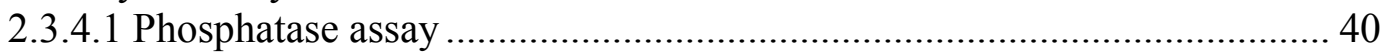

2.3.4.2 RP-HPLC analysis of FPPase catalytic products..................................... 40

2.3.4.3 Effect of inhibitors on AaFPPase activity ............................................... 41

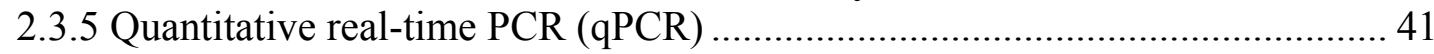

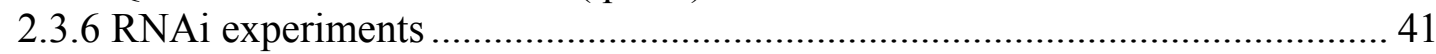

2.3.7 FPPase activity in CA extracts ............................................................... 42

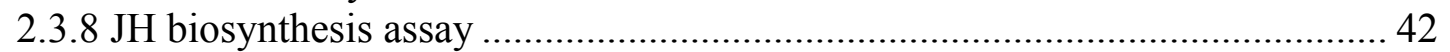

2.3.9 Secondary structure and phylogenetic analysis ............................................. 43

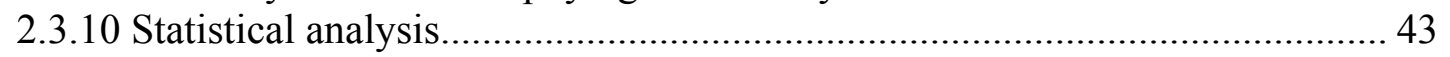

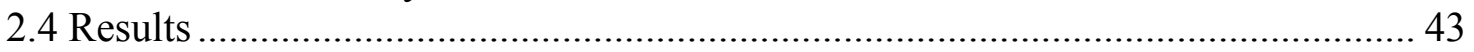

2.4.1 Identification of three A. aegypti FPPases expressed in the CA ..................... 43

2.4.2 All AaFPPases hydrolyzed p-NPP, but only AaFPPase- 1 and -2 converted

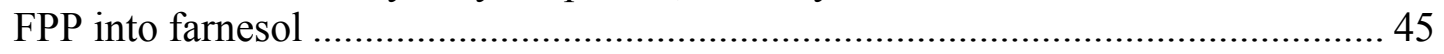

2.4.3 The CA exhibited variable FPPase activity ..................................................... 46

2.4.4 Tissue- and developmental-stage-specific expression of AaFPPases................ 47

2.4.5 Reduction of AaFPPase-1 by RNAi caused a significant decrease on JH

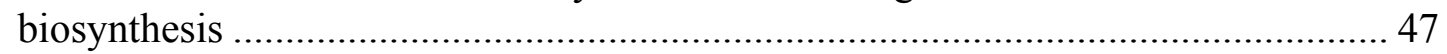

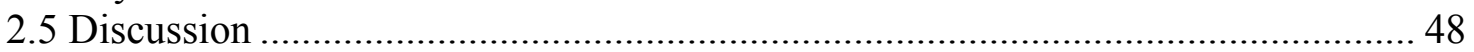

2.5.1 Molecular and functional characterization of AaFPPases expressed in the corpora allata of mosquitoes ......................................................................... 48

2.5.2. Expression of AaFPPases genes ................................................................. 49

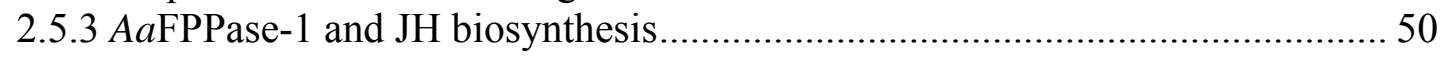

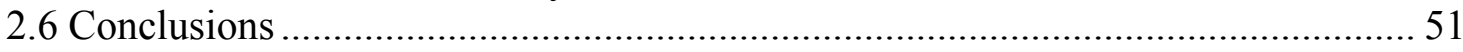

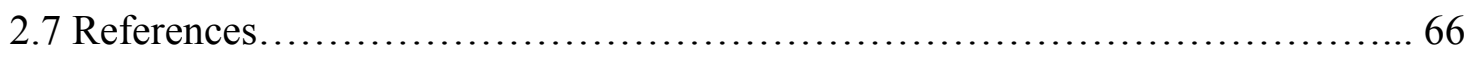

Chapter 3: Structural and biochemical characterization of a mevalonate kinase involved in juvenile hormone pathway in Aedes aegypti ............................................. 70

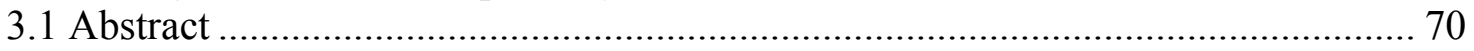

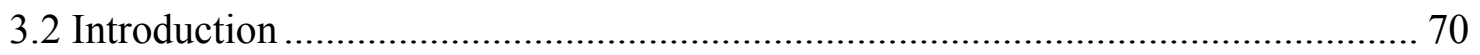

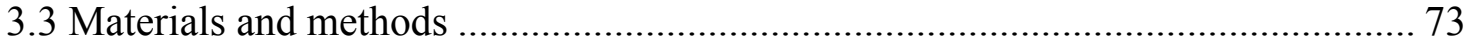

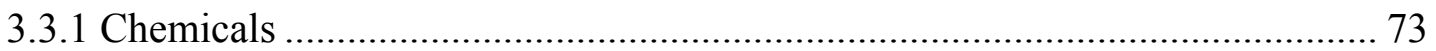

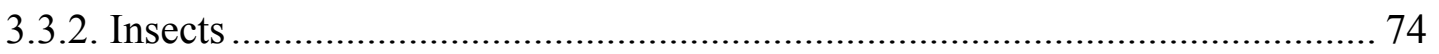

3.3.3 Secondary and tertiary structure of $A a \mathrm{MK}$..................................................... 74

3.3.4 Expression of recombinant AaMK .......................................................... 74

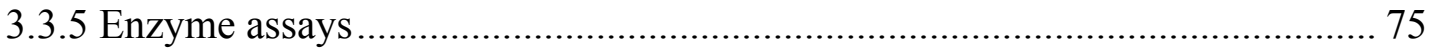

3.3.5.1 Enzyme coupled spectrophotometric assay ............................................ 75

3.3.5.2. RP-HPLC analysis of the products of MK catalysis................................ 76

3.3.6 MK activity in the crude extract (CE) of mosquitoes...................................... 76

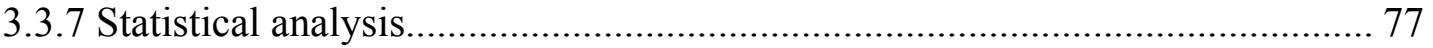

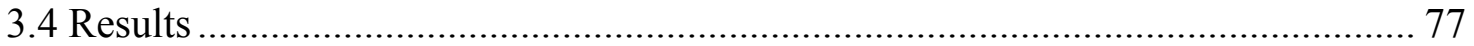

3.4.1 Molecular and structural characterization of A. aegypti mevalonate kinase .... 77

3.4.2 Expression and purification of recombinant A. aegypti mevalonate kinase..... 78

3.4.3 Mevalonate kinase activity ......................................................................... 78

3.4.4 Kinetic properties of AaMK ……………………....................................... 79 
3.4.6 Inhibition of MK activity in crude extract (CE) of mosquitoes by long chain

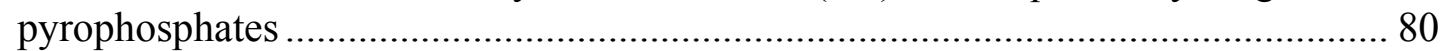

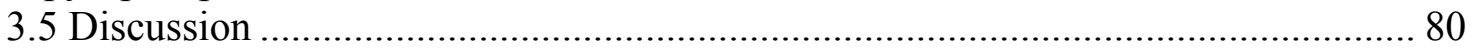

3.5.1 Structural and biochemical characterization of $A a \mathrm{MK}$............................... 80

3.5.2 Role of mevalonate kinase on JH synthesis in mosquitoes ............................ 83

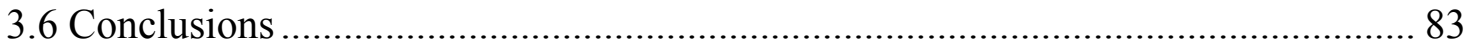

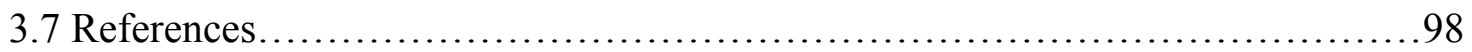

Chapter 4: Characterization of additional corpora allata enzymes involved in juvenile

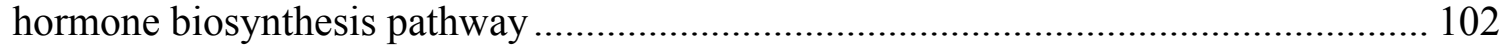

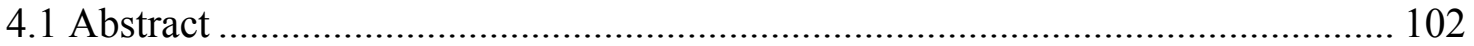

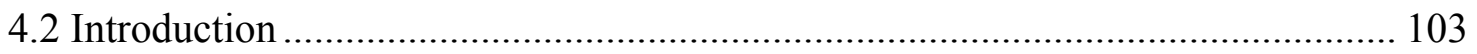

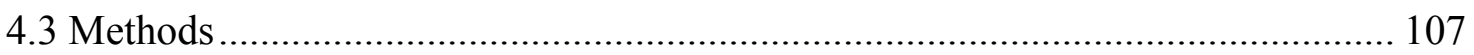

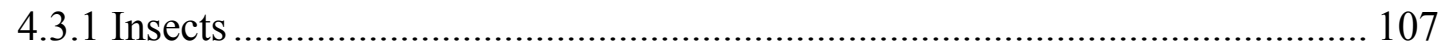

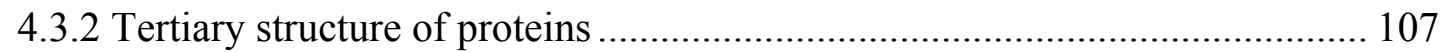

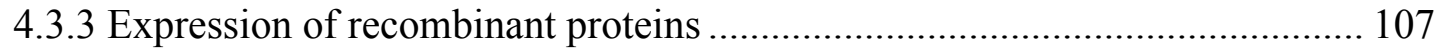

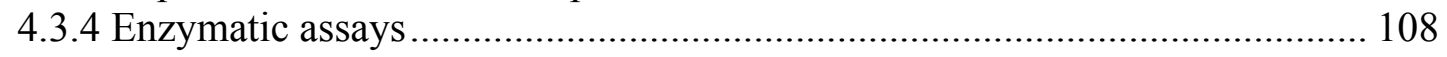

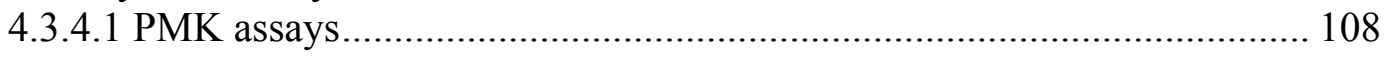

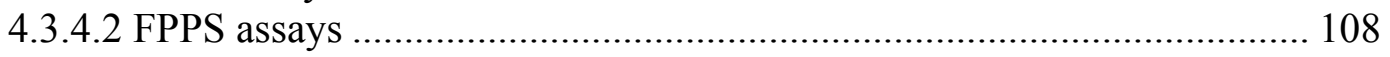

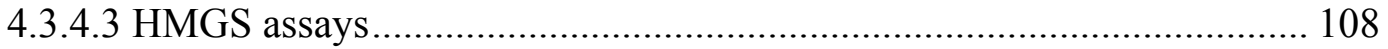

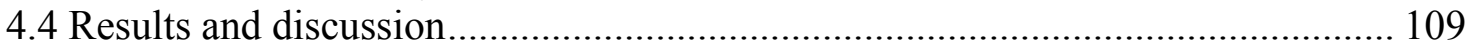

4.4.1 Structural analysis of AaPMK through molecular modelling ....................... 109

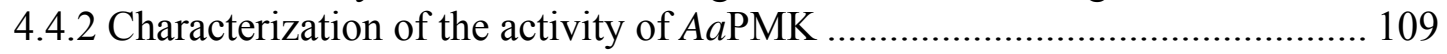

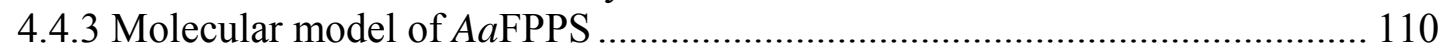

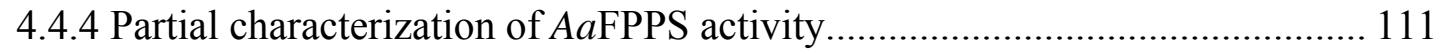

4.4.5 Characterization of the AaHMGS activity............................................... 112

4.4.6 HMGS expression and activity in the abdominal carcass of $0 \%$ Vs $20 \%$

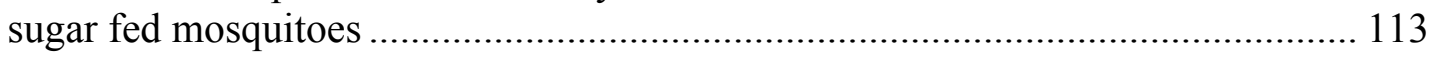

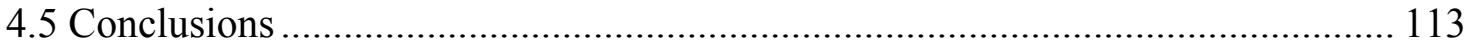

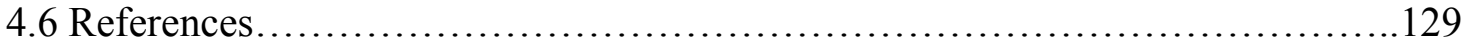

Chapter 5: Conclusions and future directions ......................................................... 133

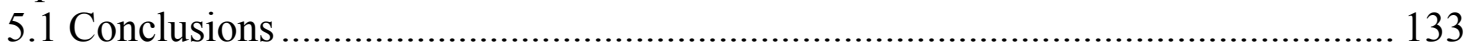

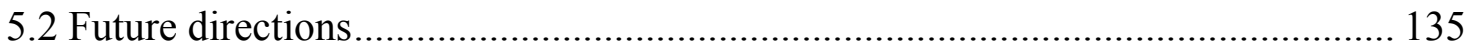

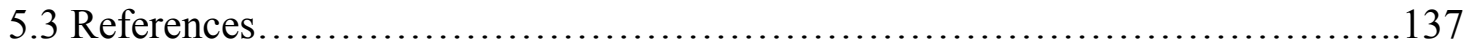

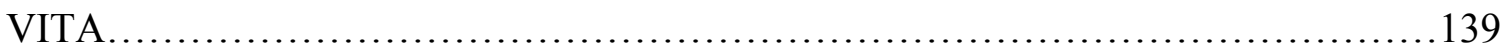




\section{LIST OF FIGURES}

FIGURE

PAGE

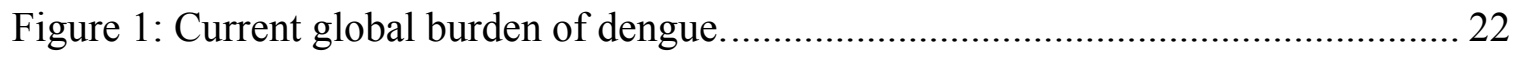

Figure 2: Map of the countries and territories where chikungunya cases have been reported. 23

Figure 3: Structure of juvenile hormones ............................................................. 24

Figure 4: Juvenile hormone III (JH III) biosynthetic pathway in mosquitoes................. 25

Figure 5: Rate of JH biosynthesis and ovarian development in female Aedes aegypti .... 26

Figure 6: Flux model of juvenile hormone synthesis............................................... 27

Figure 7: PCR analysis of the expression of eight putative phosphatase genes in the CA of adult female Aedes aegypti.

Figure 8: Homology model of the overall fold of AaFPPase-1 and amino acid sequence alignment of HAD motifs and cap domains from mosquito, fruit fly and human..... 54

Figure 9: Phylogenetic analysis of HAD superfamily sequences from insects and human. 55

Figure 10: Metal dependence and optimum $\mathrm{pH}$

Figure 11: Chromatogram of a reverse-phase high performance liquid (HPLC) analysis showing the production of farnesol from FPP by AaFPPase-1....................................59

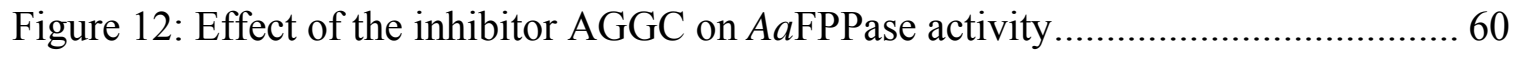

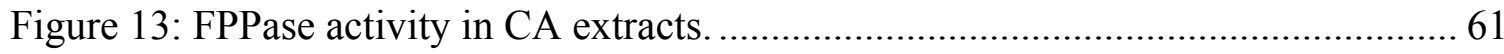

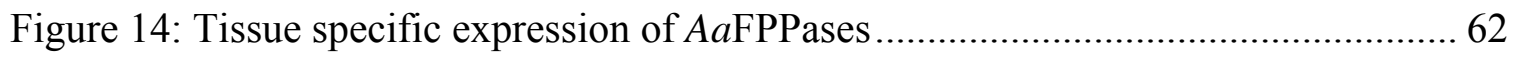

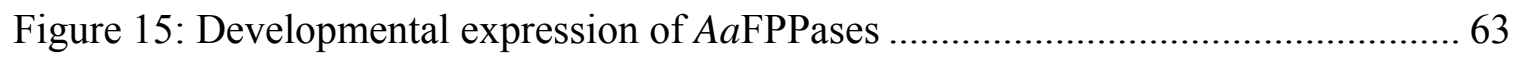

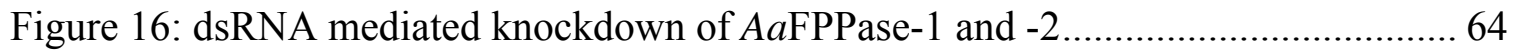

Figure 17: Schematic representation of the catalytic mechanism for AaFPPases ........... 65

Figure 18: Homology model of the overall fold of $A a \mathrm{MK}$ and its amino acid sequence. 86 
Figure 19: Amino acid sequence alignment of conserved motifs from A. aegypti MK, compared with seven different insects and rat ortholog

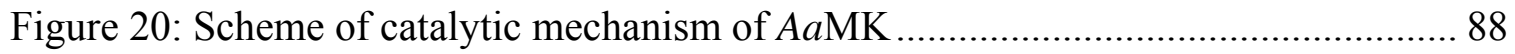

Figure 21: SDS PAGE (A) and Western blot (B) analysis of the recombinant mosquito AaMK.

Figure 22: Chromatogram of a reverse phase-HPLC analysis showing the production of PM from MA by AaMK

Figure 23: AaMK pH curve, effect of metal ions and specificity of the phosphoryl donor.

Figure 24: Inhibition of AaMK activity by GPP (A), FPP (B) and GGPP (C)

Figure 25: Inhibition of mevalonate pathway and AaMK activity by long chain pyrophosphates.

Fig. 26: Tissue specific expression of $A a \mathrm{MK}$

Fig. 27: Transcript levels (A) and enzyme activity (B) of the AaMK in developmental stage of female A. aegypti.

Figure 28: Reaction catalyzed by HMG-CoA synthase

Figure 29: Reaction catalyzed by PMK ............................................................... 116

Figure 30: Reaction catalyzed by FPP synthase. .................................................. 117

Figure 31: SDS-PAGE analysis of the recombinant mosquito AaHMGS, AaPMK and AaFPPS.

Figure 32: Homology model of the overall fold of AaPMK........................................ 119

Figure 33: Characterization of AaPMK activity ................................................ 120

Figure 34: Homology model for the overall fold of AaFPPS .................................... 122

Figure 35: Sequence alignment of the deduced amino acid sequence from insect FPPSs

Figure 36: Effect of metal cofactors on enzyme activity and product formation of AaFPPS. 
Figure 37: Characterization of AaHMGS activity .................................................... 125

Figure 38: Inhibition of AaHMGS abdominal carcass activity by hymeglusin.............. 126

Figure 39: Activity of HMGS from female AC extracts. ............................................... 127

Figure 40: Expression of HMGS mRNAs from female abdominal carcass extracts...... 128 


\section{LIST OF ABBREVIATIONS AND ACRONYMS}

AaEpox Aedes aegypti epoxidase

AaFALD Aedes aegypti farnesal dehydrogenase

AaFOLD Aedes aegypti farnesol dehydrogenase AaFPPase Aedes aegypti farnesyl phosphatase AaFPPS. Aedes aegypti farnesyl pyrophosphate synthase AGGC $\mathrm{N}$-acetyl-S-geranylgeranyl-L-cysteine AaHMGR Aedes aegypti 3-hydroxy-3-methylglutaryl-CoA reductase AaHMGS Aedes aegypti 3-hydroxy-3-methylglutaryl-CoA synthase AaIPPI Aedes aegypti isopentenyl pyrophosphate isomerase AaJHAMT Aedes aegypti juvenile hormone acid methyl transferase AaMDD Aedes aegypti mevalonate diphosphate decarboxylase AaMK Aedes aegypti mevalonate kinase AaPMK Aedes aegypti phospho mevalonate kinase

CA. Corpora allata

DMAPP .Dimethyl allyl pyrophosphate DNA Deoxyribonucleic acid DPM Diphosphomevalonate ETH Ecdysis Triggering Hormone

FA. .Farnesoic acid FAL Farnesal

FOL Farnesol 
FPP Farnesyl pyrophosphate GPP. ..Geranyl pyrophosphate HAD haloalkanoic acid dehalogenase HMG-CoA 3-hydroxy-3-methylglutaryl-CoA IPP ..Isopentenyl pyrophosphate

JH. Juvenile hormone MA Mevalonic acid MF Methyl farnesoate MVAP Mevalonate pathway PM .Phosphomevalonate RNA. Ribonucleic acid WHO World Health Organization 


\section{Chapter 1: Introduction}

\subsection{Aedes aegypti as the principal vector of dengue fever, yellow fever and chikungunya}

Aedes aegypti has been the focus of research in many areas owing to its global distribution and its involvement as a vector of dengue viruses, yellow fever and chikungunya. The viruses of these deadly diseases are passed on to humans through the bites of an infective female A. aegypti mosquito, which acquires the virus while feeding on the blood of an infected person (WHO, 2009). In the mosquito, the virus replicates in the midgut, invades the salivary gland and is ready for transmission to a new human host when the mosquito acquires a new blood meal (WHO, 2009).

Although A. aegypti is commonly known as the "yellow fever mosquito", because of the availability of an effective vaccine, yellow fever is a less of a concern worldwide (Barrett and Higgs 2007); conversely dengue fever and chikungunya are presently a major health problem in tropical and subtropical regions of the planet (Barrett and Higgs 2007; Morens and Fauci 2014).

Dengue fever is endemic in more than 100 countries, with America, South-east Asia and the Western Pacific regions been the most seriously affected (Fig. 1) (Bhatt et al., 2013). In recent years, dengue has become a major international public health concern as its transmission has increased predominantly in urban and semi-urban areas. The last five decades has seen a 30-fold increase in the incidence of dengue fever (WHO, 2012). It is now estimated that over 2.5 billion people are now at risk from dengue. Some 50-100 million new infections are estimated to occur annually with a spread to previously unaffected areas. Every year hundreds of thousands of severe cases arise, 
including 20,000 deaths (WHO 2012). There is a projection that by 2055 one third of the world's population will be exposed to the risk of dengue fever. Among the factors responsible for this increase are global warming and increases in urbanization and resistance to insecticides (Hales et al., 2002). According to the World Health Organization the economic costs of dengue fever to human society is comparable to tuberculosis, malaria, hepatitis or bacterial meningitis (Gubler, 2002).

Aedes aegypti is also the vector of the chikungunya virus, which causes a disease clinically similar to dengue, with similar epidemiologic problems caused by the lack of vaccines and specific treatments (Morens and Fauci 2014). The name 'chikungunya' which means 'to become distorted' is derived from the Kimakonde language of the Makonde people (WHO 2014; Morens and Fauci 2014). The disease was given its name because a severe musculoskeletal pain caused affected persons to walk in a stooped posture. Chikungunya outbreaks have been identified in more than 40 countries in Asia, Africa, Europe, and America (Fig. 2). By July 11, 2014, the Pan American Health Organization had reported more than 355,000 suspected and confirmed cases of chikungunya fever from different jurisdictions in the Americas, with continuing local transmission and epidemic spread (Morens and Fauci 2014)

Over the last several years, A. aegypti transmitted diseases have caused periodic epidemics in tropical as well as subtropical regions of the world. Controlling the vector is the only way to reduce the burden of these deadly diseases. There are mainly four classes of insecticides that are available for public health purposes, namely, organ chlorides, organophosphates, carbamates, and pyrethroids (WHO 2013). The extensive use of vector control insecticides has contributed to the development of insecticide resistance, 
cross resistance and adverse effects on the environment and human health. A better understanding of the biology of development and reproduction of mosquitoes might contribute to the selection of new targets for the development of better effective, safer and target specific insecticides.

Juvenile hormones (JHs) are lipophilic, acyclic sesquiterpenoids, synthesized by the corpora allata (CA), a pair of endocrine glands connected to the brain (Tobe et al., 1985). JH plays a central role in insect development, reproduction, diapause and polyphenisms (Goodman and Granger, 2005; Goodman and Cusson, 2012); therefore biosynthesis of $\mathrm{JH}$ has been considered as an attractive target for the chemical control of insects (Cusson et al., 2013). A number of recent reviews have summarized the current knowledge on JH biosynthesis in insects (Goodman and Cusson, 2012), as well as its potential as a target for insecticide discovery (Cusson et al., 2013).

\subsection{Brief history on JH research and the discovery of $\mathrm{JH}$ structures.}

Sir Vincent Brian Wigglesworth in 1934 wrote in his famous article named "The physiology of ecdysis in Rhodnius prolixus (Hemiptera). II. factors controlling molting and 'metamorphosis', this visionary statement: "The absence of metamorphosis in normal nymphs before the fifth stage must therefore be due to an inhibitory factor or hormone in the blood" (Wigglesworth, 1934). He also stated that the source of this "inhibitory hormone" was the corpora allata (CA). Afterward, Hans Piepho revealed that the formation and nature of the cuticle of the Lepidoptera Galleria was under hormonal control (Piepho, 1938). The work by Carroll Williams in the 1950s indicated that males of the Hyalophora cecropia moth contained a store of lipoidal "golden oil" with $\mathrm{JH}$ activity (William, 1956). William discovery initiated a series of studies trying to identify 
$\mathrm{JH}$ homologs in different insects, since an active extract of the $\mathrm{JH}$ was then available that worked on most insect species (Gilbert et al., 2000).

The first $\mathrm{JH}(\mathrm{JH}$ I) was identified from the lipid extract of the silkworm, Hyalophora cecropia (Roller et al., 1967) (Fig. 1). A second JH homologue (JH II) was also identified in $\mathrm{H}$. cecropia extracts, differing from JH I by the presence of a methyl group at C7 (Meyer et al., 1968). A third JH homologue (JH III) was identified from the CA of the tobacco hornworm, Manduca sexta (Judy et al., 1973). JH III is the most ubiquitous homologue in insects, and also the $\mathrm{JH}$ form present in Aedes aegypti (Schooley and Baker, 1985). JH 0 and 4-methyl JH I (iso JH 0) were identified from eggs of M. sexta (Bergot et al., 1981) (Fig. 1). A JH III form with a second epoxide substitution at $\mathrm{C} 6, \mathrm{C} 7$ ( $\mathrm{JH}$ III bisepoxide or $\mathrm{JHB}_{3}$ ) is present in Drosophila melanogaster (Richard et al., 1989) (Fig. 1). Finally, a skipped bisepoxide ( $\left.\mathrm{JHSB}_{3}\right)$, with a second epoxide substitution at $\mathrm{C} 2, \mathrm{C} 3$, rather than at $\mathrm{C} 6, \mathrm{C} 7$ as in $\mathrm{JHB}_{3}$, was identified from the pentatomid, Plautia stali (Kotaki et al., 2009) (Fig. 1). JH analogs (JHA) such as methoprene, pyriproxifen and fenoxycarb are compounds that mimic the action of JH and have been employed as control agents for mosquitoes, flies, stored-product pests, fleas and fire ants (Goodman and Cusson, 2012) (Fig. 1).

\subsection{Juvenile hormone biosynthesis}

\subsubsection{Biosynthetic pathway}

The JH biosynthetic pathway can be divided into two distinct biosynthetic units, the early steps and the late steps (Fig. 2) (Belles et al., 2005). The early steps follow the mevalonate pathway (MVAP) to form farnesyl pyrophosphate (FPP). In the MVAP, acetyl-CoA undergoes a series of enzymatic reactions to form the 5-carbon compound 
isopentenyl-pyrophosphate (IPP). A sequential head to tail condensation of three $5 \mathrm{C}$ units is used to synthesize the 15-carbon farnesyl pyrophosphate (FPP) (Belles et al., 2005). FPP is a precursor of cholesterol in many organisms. Insects do not synthesize cholesterol de novo because they lack the genes encoding the enzymes required for the production of cholesterol from FPP, including squalene synthase and other subsequent enzymes of the sterol branch (Clark and Bloch, 1959). FPP is shunted into other pathways in insects, including synthesis of ubiquinone and dolichol, protein prenylation, and pheromone synthesis.

The late steps of JH biosynthesis (JH-branch) include the hydrolysis of FPP to farnesol (FOL) (Cao et al., 2009; Nyati et al., 2013), which is then successively oxidized to farnesal (FAL) by an alcohol dehydrogenase, and to farnesoic acid (FA) by an aldehyde dehydrogenase (Mayoral et al., 2009a; Rivera-Perez et al., 2013) (Fig. 2). The last two steps vary in different insect orders. In Lepidoptera, a C-10, 11 epoxidation by a P450 monooxygenase converts the FA to the epoxy acid ( $\mathrm{JH}$ acid or JHA), which is then methylated by methyltransferase (JHAMT) to form the methyl ester. In Orthoptera and Dictyoptera, epoxidation follows methylation and this is also the case in mosquitoes (Defelipe et al., 2012; Noriega, 2014).

Expression of the first three enzymes of the JH-branch, namely, FPP phosphatase (FPPase), farnesol dehydrogenase (FOLD) and farnesal dehydrogenase (FALD), is not restricted to the CA (Nyati et al., 2013; Mayoral et al., 2009a; Rivera-Perez et al., 2013); but are expressed in several tissues, where they might play a significant role in cellular activities such as proliferation, apoptosis, signal transduction, and vesicular transport (Joo and Jetten, 2010; Pechlivanis and Kuhlmann, 2006). In contrast, the last two enzymes of 
the JH-branch, the JHAMT and epoxidase (Epox) are likely exclusive for JH biosynthesis and therefore are highly expressed in the CA (Mayoral et al., 2009b; Nouzova et al., 2011).

\subsubsection{Enzymes: Biochemical characterization}

\subsubsection{Acetoacetyl-CoA thiolase (EC 2.3.1.9)}

It catalyzes the condensation of two molecules of acetyl CoA to form acetoacetyl CoA. Its activity is yet to be characterized in insects.

\subsubsection{HMG-CoA synthase (EC 2.3.3.10)}

HMG-CoA synthase (HMGS) catalyzes the condensation of acetoacetyl-CoA and acetyl-CoA into 3-hydroxy-3-methylglutaryl-coenzyme A (HMG-CoA). In insects HMGS has been biochemically characterized from the cockroach, Blattella germanica (Buesa et al., 1994; Casals et al., 1996) and A. aegypti (chapter 4). In both insects HMGS does not require a co-factor for activity; however the activity of the recombinant enzymes was enhanced by adding $\mathrm{Mg}^{2+}$ (Buesa et al., 1994; chapter 4). Hymeglusin, a specific $\beta$ lactone inhibitor of the vertebrate HMGS (Greenspan et al., 1987), is an inhibitor of the HMGS enzyme activity in crude extracts from thorax and abdominal carcass of $A$. aegypti (chapter 4).

\subsubsection{HMG-CoA reductase (EC 1.1.1.34)}

HMG-CoA reductase (HMGR) reduces HMG-CoA to mevalonic acid. The activity of the HMGR has been characterized in two cockroach species: Blattella germanica (Casalet al., 1996) and Diploptera punctata (Feyereisen and Farnsworth, 1987). The activity of HMGR strictly depends on NADPH, and it is competitively inhibited by mevinolin (Feyereisen and Farnsworth, 1987). 


\subsubsection{Mevalonate kinase (EC 2.7.1.36)}

Mevalonate Kinase (MK) catalyzes the phosphorylation of mevalonic acid into phosphomevalonate (PM). MK is a member of the "sugar kinase family" that includes enzymes such as galactokinase, homoserine kinase, mevalonate kinase, and phosphomevalonate (GHMP) kinase (Bork et al., 1993; Cheek et al., 2002). In insects, MK has been characterized only in A. aegypti (AaMK) (Nyati et al., in prep). The catalytic activity of $\mathrm{AaMK}$ increases in a dose response manner when $\mathrm{Mg}^{2+}$ is added as a cofactor. AaMK can also utilize $\mathrm{Mn}^{2+}$ and $\mathrm{Co}^{2+}$ as cofactors, but the activity is much higher with $\mathrm{Mg}^{2+}$ (Nyati et al., in prep). The activity of AaMK is strongly inhibited by long chain isoprenoids such as geranyl-geranyl pyrophosphate (GGPP) (20 C units), farnesyl pyrophosphate (FPP) (15 C units) and geranyl pyrophosphate (GPP) (10 C units).

\subsubsection{Phosphomevalonate kinase (EC 2.7.4.2)}

Phosphomevalonate kinase (PMK) catalyzes the cation-dependent reversible reaction of PM and ATP to form diphosphate mevalonate (DPM) and ADP. Metazoans PMKs are not related to the GHMP kinases, but exhibit the typical fold of the nucleoside monophosphate (NMP) kinase family members (Smith and Mushegian, 2000). In insects, PMK has been characterized only in A. aegypti (AaPMK) (Chapter 4).

\subsubsection{Mevalonate diphosphate decarboxylase (EC 4.1.1.33)}

Mevalonate diphosphate decarboxylase (MDD) catalyzes ATP dependent decarboxylation of DPM to form isopentenyl pyrophosphate (IPP), releasing ADP and $\mathrm{CO}_{2}$ as byproducts. Like MKs, MDDs are also classified as members of the GHMP kinase family (Bork et al., 1993; Cheek et al., 2002). Although MDDs have been cloned 
in a variety of insects, and the crystal structure of non-insect proteins are known (Miziorko, 2011), its relation to JH biosynthesis is poorly characterized. Fluoromevalonate is a potent inhibitor of MDD in several lepidopteran species; inhibiting the metabolism of MA to JH very efficiently in Manduca sexta (Quistad et al., 1981).

\subsubsection{Isopentenyl diphosphate isomerase (EC 5.3.3.2)}

Isopentenyl diphosphate isomerase (IPPI) catalyzes the reversible conversion of IPP into dimethylallyl pyrophosphate (DMAPP). IPPI has been biochemically characterized from A. aegypti (Diaz et al., 2012) and two lepidopteran species: Choristoneura fumiferana and M. sexta (Sen et al., 2012). IPPIs belong to the Nudix hydrolase superfamily. IPPI activity is enhanced by $\mathrm{Mg}^{2+}$ or $\mathrm{Mn}^{2+}$ and inhibited by iodoacetamide in A. aegypti (Diaz et al., 2012), and by ammonium diphosphates in lepidoptera (Sen et al., 2012).

\subsubsection{FPP synthase (EC 2.5.10)}

FPP synthases (FPPSs) are short-chain prenyltransferases that catalyze two sequential coupling of IPP in a head to tail manner. The alkylation of IPP by DMAP to produce GPP occurs first; that reaction is followed by the alkylation of IPP by GPP to yield FPP. In the beetle Phaedon cochleariae, FPPS shows an unusual product regulation mechanism; it alters the chain length of its products depending on the cofactor present. The FPPS yields $\mathrm{C}_{10}$-GPP in the presence of $\mathrm{Co}^{2+}$ or $\mathrm{Mn}^{2+}$, whereas it produces the longer $\mathrm{C}_{15}$-FPP in the presence of $\mathrm{Mg}^{2+}$ (Frick et al., 2013). A similar mechanism has been observed in A. aegypti (Rivera-Perez et al., in prep). Inhibitors of the FPPSs include bisphosphonates such as BPH-461, BPH-527 and BPH-678 (Cusson et al., 2013). 


\subsubsection{FPP phosphatase (EC 3.1.7.6)}

FPP phosphatases (FPPases) catalyze the hydrolysis of FPP into FOL. The first insect FPPases were characterized in Drosophila melanogaster (Cao et al., 2009) and A. aegypti (Nyati et al., 2013). The FPPases belong to the NagD haloalkanoic acid dehalogenase family (HAD). A. aegypti FPPase (AaFPPase) efficiently hydrolyzes FPP and GPP but not IPP (Nyati et al., 2013). Insect FPPases increase their catalytic activity in a dose response manner when $\mathrm{Mg}^{2+}$ is used as a cofactor, and it are strongly inhibited by N-acetyl-S-geranylgeranyl-L-cysteine (AGGC) (Cao et al., 2009; Nyati et al., 2013).

\subsubsection{Farnesol dehydrogenase (FOLD)}

Two types of enzymes have been proposed for the oxidation of FOL into FAL: 1) a flavin/iron dependent alcohol oxidase activity was characterized from a CA homogenate of Manduca sexta (Sperry and Sen, 2001); and 2) a short chain alcohol dehydrogenase (SDR) was molecularly and biochemically characterized in the CA of $A$. aegypti (Mayoral et al., 2009a). The oxidation of farnesol by M. sexta CA extracts was weakly inhibited by 1, 10-phenanthroline (Sperry and Sen, 2001). In A. aegypti NADP ${ }^{+}$ was absolutely required for FOLD activity, and $\mathrm{NAD}^{+}$or $\mathrm{FAD}^{+}$did not substitute (Mayoral et al., 2009a).

\subsubsection{Farnesal dehydrogenase (FALD) (EC 1.2.1.B9)}

A fatty aldehyde dehydrogenase (AaALDH3) that oxidizes FAL into FA was identified in CA of A. aegypti (Rivera-Perez et al., 2013). The AaALDH3 is structurally and functionally a $\mathrm{NAD}^{+}$-dependent class $3 \mathrm{ALDH}$ with orthologues in many insect species (Rivera-Perez et al., 2013). 


\subsubsection{JH acid methyltransferase (JHAMT) (EC 2.1.1.15)}

Recombinant JH acid methyltransferase (JHAMT) transfers a methyl group from S-adenosylmethionine (SAM) into FA to produce MF, as well as into JHA to generate JH III in all the insect species studied (Shinoda and Itoyama, 2003; Minakuchi et al., 2008; Niwa et al.,2008; Mayoral et al., 2009b). Homology modeling and docking simulations confirmed that JHAMT is capable to methylate both FA and JHA (Defelipe et al., 2011). JHAMT is an enzyme highly specific to insects hence, JHAMT specific inhibitors are expected to be excellent candidates for safe insect growth regulators.

\subsubsection{MF epoxidase (Epox) (3.6.2.4)}

MF Epox is generally consider as the last enzyme of the JH biosynthetic pathway, that catalyzes epoxidation of $\mathrm{MF}$ into $\mathrm{JH}$ in most of insects with the exception of Lepidoptera (Bhaskaran et al., 1986), Drosophila (Moshitzky and Applebaum, 1995) and Hemiptera (Kotaki et al., 2009). MF Epox uses NADPH as a cofactor, and is strongly inhibited by 1, 5-disubstituted imidazoles such as TH27 and KK96 (1-Isobutyl-5-[3[[(E)-3,7-dimethyl-2,6-octadienyl]oxy]phenyl]-1H-imidazole) (Helvig et al., 2004). Recombinant D. punctata EPOX (CYP15A1) shows higher affinity for MF, which it converted to JH III and cannot catalyze FA into JHA (Helvig et al., 2004). On the other hand Bombyx mori Epox exhibits at least 18-fold higher activity for FA than MF (Daimon et al., 2012). Therefore, the order of the methylation/epoxidation reactions depends upon the Epox substrate specificity (Defelipe et al., 2011). In Lepidoptera, Epox has a higher affinity for FA than JHAMT, so epoxidation precedes methylation; whereas in many other insects Epox cannot metabolize FA, so methylation precedes epoxidation. 


\subsection{JH functions}

\subsection{1 $\mathrm{JH}$ is one of the key hormones regulating growth and metamorphosis of insects}

Two key hormones are responsible for the regulation of growth and metamorphosis in insects; JH and 20-hydroxyecdysone (20E) (Nijhout, 1994). Ecdysone is secreted by the prothoracic glands of preimaginal stages and the ovary of adult insects. Ecdysone is converted in periphery tissues to 20E (Goodman and Granger 2005; Hiruma and Kaneko 2013). Periodic pulses of 20E induce molts, and the nature of the molt depends on $\mathrm{JH}$. The presence of $\mathrm{JH}$ ensures that the molt will produce another immature instar (nymphal in hemimetabolous and larval in holometabolous insects). The reduction in $\mathrm{JH}$ titers in the final nymph instars or larvae stages induces metamorphosis (Nijhout, 1994). Except for the higher Diptera, treatment of final-instar nymphs, larvae, or pupae with JH or JHA causes repetition of that stage (Goodman and Cusson, 2012). Conversely, experimental removal of $\mathrm{JH}$ at earlier instars leads to precocious metamorphosis. In some species, such as the silkworm, Bombyx mori (Tan et al., 2005) and the red flour beetle, Tribolium castaneum (Minakuchi et al., 2008), depletion of JH can even yield perfect miniature pupae and adults. For its ability to prevent differentiation without interfering with growth, JH was termed as a 'status quo' hormone (Goodman and Granger, 2005 and Dubrovsky, 2005).

\subsection{2 $\mathrm{JH}$ assesses nutritional information to regulate reproduction in mosquitoes}

In insect's life one of the critical components is to correctly distribute nutritional reserves among survival, growth and reproduction (Boggs, 2009; Clifton and Noriega, 2011). JH is part of a transduction system that evaluates nutritional information and controls the development of ovaries in insects (Noriega, 2004). There are three major 
sources of nutrients that are used by female A. aegypti during the three different phases of ovarian development. Reserves acquired from preimaginal stages, nectar feeding and blood meal are respectively used for the previtellogenesis (PVG), resting stage and vitellogenesis (VG) phases of ovary development (Briegel, 1990; Klowden, 1997; Noriega, 2004; Zhou et al., 2004a, 2004b). In newly eclosed adult female A. aegypti JH synthesis and ovarian previtellogenic maturation are activated only if teneral nutritional reserves are elevated (Caroci et al., 2004). Later, after previtellogenic maturation has been completed, $\mathrm{JH}$ mediates reproductive trade-offs in resting stage mosquitoes in response to nutrition (Clifton and Noriega, 2012). An adult female A. aegypti shows dynamic changes in JH biosynthesis, and regulation of the CA activity are quite different in the previtellogenesis, ovarian resting stage and vitellogenesis phases (Rivera-Perez et al., 2014) (Fig. 3). Four distinct nutritional-dependent stages of CA activity have been described in female A. aegypti: inactive, active, modulated and suppressed CA on the basis of the rates of JH biosynthesis (Rivera-Perez et al., 2014) (Fig. 3).

JH biosynthesis rate changes correspond well with the changes in transcript levels for most of the JH biosynthetic enzymes. Transcript levels are very low in early pupae (Nouzova et al., 2011) and JH synthesis rates are undetectable in pupae 24 and $12 \mathrm{~h}$ before adult eclosion (Rivera-Perez et al., 2014) (Fig. 3). Subsequently, in the last 6-8 $\mathrm{h}$ before adult emergence transcript levels for the biosynthetic enzymes commence to rise, the pupal CA becomes "competent" and starts to synthesize JH (Nouzova et al., 2011). Although the CA of the newly eclosed adult female is fully competent; for the next 10-11 $\mathrm{h}$ it synthesizes relatively low levels of JH (10 fmol/h) (Rivera-Perez et al., 2014) (Fig 3). The brain of the mosquito plays a key role in sensing the nutrients and regulating the 
activity of CA, since decapitation during $12 \mathrm{~h}$ after emergence prevents the rise in $\mathrm{JH}$ levels (Hernandez-Martinez et al., 2007). A sharp increase in JH synthesis is observed 12 $\mathrm{h}$ after adult emergence, which conveys information on teneral nutritional reserves and gives signal for the previtellogenic maturation of the ovaries. The process of "activation" of CA is very fast and short lasted; JH synthesis increases from $10 \mathrm{fmol} / \mathrm{h}$ to almost 100 $\mathrm{fmol} / \mathrm{h}$ in $2 \mathrm{~h}$, and decreases to less than $40 \mathrm{fmol} / \mathrm{h}$ in the next $2 \mathrm{~h}$; remaining at this relatively high and constant rate until $24 \mathrm{~h}$ after emergence (Rivera-Perez et al., 2014) (Fig. 3). Later, during the ovarian resting stage, female mosquitoes are capable of synthesizing different rates of $\mathrm{JH}$ depending upon the amount of nutrients available. A. aegypti that were fed on a restricted 3\% sucrose diet had reduced JH synthesis (RiveraPerez et al., 2014) (Fig. 3). Finally at the VG phase; $24 \mathrm{~h}$ after blood feeding there is a suppression of JH synthesis (Li et al., 2003a; Rivera-Perez et al., 2014).

\subsection{Regulation of JH biosynthesis}

\subsubsection{Role of the insulin/TOR signaling pathway}

The insulin/TOR signaling pathways play a central role in the transduction of nutritional signals into cell growth and metabolism in almost all eukaryotic cells (Howell and Manning, 2011; Siddle, 2012). In D. melanogaster specific silencing of the insulin receptor (InR) in the CA completely suppresses HMG-CoA expression and renders a JHdeficient phenotype (Belgacem and Martin, 2007). In addition, D. melanogaster InR mutants have reduced JH synthesis (Tu et al., 2005). In Culex pipiens, the ability to enter into overwintering diapause is regulated by JH (Sim and Denlinger, 2008). Silencing the InR by RNAi or the downstream FOXO protein (fork head transcription factor) in $C$. pipiens leads to a diapause phenotype (Sim and Denlinger, 2008). 
There are eight insulin like peptides (ILPs) reported in the genome of A. aegypti; three of them (ILP 1, 3 and 8) show expression specifically in brains of adult females (Riehle et al., 2006). ILP3 shows the highest affinity binding for the A. aegypti insulin receptor (InR); and stimulates egg maturation by activating the insulin signaling pathway in the ovaries (Brown et al., 2008). Insulin receptor tyrosine kinase activity and phosphatidylinositol 3-kinase mediate mosquito ILPs action (Riehle and Brown, 1999). Selective activators and inhibitors of insulin signaling cascades have strong effects on insulin-regulated physiological processes in mosquitoes (Riehle and Brown, 1999); for example, knockdown of the A. aegypti "phosphatase and tensin homolog" (AaegPTEN) affects insulin signaling (Arik et al., 2009). Bovine insulin shows a strong and fast stimulation of JH synthesis by mosquito CA-CC dissected from 1 or 3 day old sugar fed females (Perez-Hedo et al., 2013). Incubation of CA-CC with LY294002, an inhibitor of insulin signaling pathway resulted in a strong decrease in JH synthesis (Perez-Hedo et al., 2013). JH biosynthetic transcript levels and JH synthesis were reduced by the systemic depletion of TOR by RNAi, as well as by administration of the TOR modulator rapamycin (Perez-Hedo et al., 2013).

In A. aegypti starvation decreases JH synthesis via a decrease in insulin signaling in the CA. Starvation results in up regulation of the insulin receptor, which increases CA insulin sensitivity and might "prime" the gland to respond rapidly to increases in insulin levels after feeding resumption (Perez-Hedo et al., 2014). During this response to starvation the synthetic potential of the CA remained unaffected, and the gland rapidly and efficiently responds to insulin stimulation by increasing $\mathrm{JH}$ synthesis to rates similar to those of CA from non-starved A. aegypti (Perez-Hedo et al., 2014). 


\subsection{2 $\mathrm{JH}$ biosynthesis regulation by allatotropins and allatostatins}

Allatotropins (ATs) are neuropeptides that stimulate CA activity, while allatostatins (AST) inhibit JH synthesis (Goodman and Cusson, 2012). The insect AT receptor belongs to a family of G-Protein-Coupled Receptors (GPCRs) orthologues of the vertebrate orexin/hypocretin receptors (Yamanaka et al., 2008; Horodyski et al., 2011; Vuerinckx et al., 2011; Nouzova et al., 2012). The Bombyx mori AT receptor (BmATr) is not expressed in the $\mathrm{CA}$, but in the corpora cardiaca $(\mathrm{CC})$; and it was suggested that AT inhibits the release of short Neuropeptide F, which in its turn inhibits JH synthesis. Thus AT exerts an indirect allatotropic effect by "derepression" (Yamanaka et al., 2008) which has not been tested in mosquitoes or additional insect species. Unlike BmATr, A. aegypti AT receptor (AeATr) is expressed in the CA (Nouzova et al., 2012). The pattern of changes of AeATr mRNA in the CA resembles the changes in $\mathrm{JH}$ biosynthesis, and it was suggested that the AeATr might play a role in the regulation of $\mathrm{JH}$ synthesis in mosquitoes (Nouzova et al., 2012); however its exact roles in vivo and the mechanisms of action of AT still need to be elucidated.

Insects AST can be grouped into three families, cockroach allatostatins (YXFGLamide or type-A), cricket allatostatins (W2W9 or type-B), and Manduca allatostatins (PISCF or type-C) (Stay et al., 1994; Bendena et al., 1999; Stay and Tobe, 2007). The receptors for the three ASTs (A, B and C) also belong to the GPCR family with vertebrate orthologues. The AST-A receptors are related to the vertebrate galanin receptors (Kreienkamp et al., 2002), the AST-B receptors to the bombesin receptors (Johnson et al., 2003), and the AST-C receptors show similarity to the somatostatin/opioid receptors (Kreienkampet al., 2006, Mayoral et al., 2010). AST-A 
inhibits JH synthesis in Diploptera punctata CA, by reducing the availability of acetylCoA from glucose or amino acid metabolism, although the incorporation of acetate into the JH pathway remains unaffected (Sutherland and Feyereisen, 1996). Thus a major target of AST-A could be either the transport of citrate across the mitochondrial membrane or/and the cleavage of citrate to yield cytoplasmic acetyl-CoA (Sutherland and Feyereisen, 1996). Similar results have been described for the action of AST-C in mosquitoes. Aedes aegypti AST-C (AeaAST-C) showed no inhibitory activity in the presence of any of the intermediate precursors of JH indicating that the AeaAST-C target is located before the entry of acetyl-CoA in the pathway (Nouzova et al., in prep). Stimulation experiments using different sources of carbon (glucose, pyruvate, acetate and citrate) suggest that AST-C acts after pyruvate is transformed to citrate in the mitochondria. In vitro inhibition of the citrate mitochondrial carrier (CIC) mimicked the

effect of AeaAST-C, and was overridden by addition of citrate or acetate. Treatment of the CA with a calcium modulator, thapsigargin (inhibitor of SERCA pumps) superseded AeaAST-C inhibition, suggesting an involvement of $\mathrm{Ca}^{2+}$ in the AeaAST-C signaling pathway (Nouzova et al., unpublished manuscript).

\subsubsection{Regulation of $\mathrm{JH}$ synthesis by 20-hydroxyecdysone and ecdysis triggering hormone}

Twenty-hydroxyecdysone (20E) is a steroid hormone that controls molting, metamorphosis and oogenesis in mosquitoes (Margam et al., 2006; Attardo et al., 2005). 20E regulates JH synthesis in Bombyx mori (Gu and Chow 1996, Kaneko et al., 2011), possibly by means of a direct control on the expression of some of the JH biosynthetic enzymes (Hiruma and Kaneko 2013). 
Ecdysis triggering hormone $(\mathrm{ETH})$ is a small C-terminally amidated peptide that is released into the hemolymph to activate pre-ecdysis and ecdysis motor programs in the central nervous system (CNS) (Zitnan and Adams, 2012). Specialized endocrine cells called Inka cells synthesize ETH (Adams et al., 2006; Zitnan et al., 2007). The expression of the ETH gene is regulated by 20E (Zitnan and Adams, 2012). ETH receptors are expressed in the CA of the moths Bombyx mori and Manduca sexta (Yamanaka et al., 2008); so it has been suggested that ETH could play a role in the regulation of JH biosynthesis (Yamanaka et al., 2008). In A. aegypti, in vitro stimulation of the pupal CA with ETH resulted in an increase in JH synthesis; conversely silencing of A. aegypti ETH receptor in pupa resulted in reduced JH synthesis by the CA of one day old adult females (Areiza et al., 2014). There is an increase in the activity of JHAMT when A. aegypti CA is stimulated with ETH (Areiza et al., 2014). ETH increases JH synthesis in A. aegypti by mobilizing calcium from intracellular stores (Areiza et al., 2014).

\subsubsection{Flux control of JH synthesis rate}

The rate of JH biosynthesis is controlled by the rate of flux of isoprenoids in the pathway, which is the outcome of a complex interplay of changes in precursor pools, enzyme levels and external regulators (Fig. 4) (Nouzova et al., 2011). Changes in the nutritional status in female mosquitoes, as well as the manipulation of individual precursor pool concentrations (e.g. FOL, FAL and FA) affect the rate of JH biosynthesis (Fig. 4) (Nouzova et al., 2011; Rivera-Perez et al., 2014). There is a coordinated expression of JH biosynthetic enzymes in female pupae and adult mosquito. Positive correlations between JH synthesis and transcripts levels for the JH biosynthetic enzymes 
suggest that a coordinated regulation in the transcription of the genes encoding $\mathrm{JH}$ biosynthetic enzymes is at least partially responsible for the changes of JH biosynthesis in the CA of mosquitoes (Nouzova et al., 2011). To understand how regulators modify $\mathrm{JH}$ synthesis, it is important to know their effect on the changes in the levels of all enzymes and precursor pool sizes.

The 13 distinct enzymatic steps of the JH synthetic pathway are arranged in an obligatory sequence. Each product represents the substrate for the next downstream enzyme. Enzymes are connected by metabolite pools that are common to them, for example FAL is the product of the FOLD and the substrate for FALD which shows that the pools and fluxes are critical variables in JH regulation. Fluxes are distributed to all the enzymes of the pathway rather than restricting to the rate limiting enzyme but the control of the flux differ widely in a pathway and the questions of its control cannot be answer by looking at one step in isolation or even each step in isolation (Kacser and Burns 1973). It has been postulated that in a pathway with multiple enzymes all the enzymes are in excess, so that individual amounts can be significantly reduced without considerable effect on the flux (Noriega, 2014). For example in D. punctate, inhibition of HMGR activity by one third resulted in less than $15 \%$ inhibition of JH III synthesis (Sutherland and Feyereisen, 1996), showing that this enzyme is in excess and has a low control coefficient on JH synthesis. Rate limiting bottlenecks have been proposed at different enzymatic steps in both the MVAP and JH-branch in the CA of different insects, for e.g. the activity of AaMK is controlled by feedback regulation of metabolites such as FPP and GPP operating in the downstream portions of the pathway (Nyati et al, unpublished manuscript). The low enzymatic activity of FPPase and FALD could limit the flux of 
precursors and JH biosynthesis in the CA of blood-fed mosquitoes (Nyati et al., 2013; Rivera-Perez et al., 2012). In A. aegypti five enzymes: acetoacetyl-CoA thiolase, PMK, FPPase, FOLD and FALD presented overall low levels of expression in the CA (Nouzova et al., 2011; Rivera-Perez et al., 2012; Nyati et al., 2013). Under some conditions any of these enzymes could become rate limiting or bottleneck. In contrast recent studies suggest that there are multiple regulatory points in the pathway and they might change in different physiological stages (Rivera-Perez et al., 2014).

Addition of exogenous precursors such as FPP, FOL, FAL, FA, and MF stimulate $\mathrm{JH}$ synthesis in CA dissected from female mosquitoes. Stimulation of JH synthesis is independent of their spontaneous $\mathrm{JH}$ biosynthetic activity and rate of stimulation is significantly lower in suppressed CA with low JH biosynthetic activity (Nouzova et al., 2011). It is often puzzling to note that even with high endogenous pools of FOL, FAL and FA there is a limited JH synthesis in the newly emerged mosquitoes CA which can strongly be stimulated by exogenous supply of these precursors (Nouzova et al., 2011; Rivera-Perez et al., 2014). These results suggest differences in the channeling of endogenous and exogenous pools of $\mathrm{JH}$ precursors. Although the transcripts and catalytic activities of MVAP and JH branch enzymes are well coordinated with JH biosynthesis, the global fluctuations in the metabolite pools sizes are inversely related in the two pathways (Rivera-Perez et al., 2014; Nouzova et al., 2011). Analysis of the precursor pool size revealed remarkable modifications in the fluxes in the MVAP and JH-branch during first $24 \mathrm{~h}$ after adult eclosion; a 10-fold increase in JH synthesis rates by $12 \mathrm{~h}$ caused a striking depletion of all MVAP metabolite pools, with some of them replenished by $24 \mathrm{~h}$ (Rivera-Perez et al., 2014). In addition, there are examples of a reversal of the 
flux in the MVP and its branches. Phosphatase activities converting DPM back into PM and PM back into MA, a kinase activity converting FOL back into FPP, and a reductase activity converting FAL back into FOL have been previously described in plants, animals and in the CA of mosquitoes (Thai et al., 1999; Rizzo and Craft, 2000; Rivera-Perez et al., 2013). Altogether these results show that the regulation of the JH biosynthesis pathway is very complex, and further studies will be necessary to reveal what factors restrict the flux into JH III at specific physiological conditions.

\subsection{Dissertation objectives}

Juvenile hormone $(\mathrm{JH})$ plays important roles in the regulation of development, metamorphosis and reproduction of insects (Nijhout, 1994; Goodman and Cusson 2012; Noriega, 2014). The study of JH is very challenging because of the minute size of the CA and the "sticky" and unstable nature of $\mathrm{JH}$. To understand the rate limiting steps and regulatory points on the regulation of $\mathrm{JH}$ synthesis we need to complete the molecular and biochemical characterization of the 13 enzymes involved in the pathway. Four CA enzymes IPP isomerase (Diaz et al., 2012), farnesol dehydrogenase (Mayoral et al., 2009a), farnesal dehydrogenase (Rivera-Perez et al., 2013) and methyl transferase (Mayoral et al., 2009b) have already been characterized in our laboratory. The goal of my dissertation research is to characterize five additional CA enzymes namely, HMG-CoA synthase (HMGS), mevalonate kinase (MK), phosphomevalonate kinase (PMK), farnesyl pyrophosphate synthase (FPPS) and farnesyl pyrophosphatase (FPPase), and to establish their role in the regulation of $\mathrm{JH}$ synthesis. 


\subsection{Dissertation organization}

In the first chapter of my dissertation I introduce my research model organism, Aedes aegypti. The mosquito is an important vector of three deadly human viral diseases: dengue fever, yellow fever and chikungunya. The study of the hormonal regulation of development and reproduction, including a better characterization of the enzymes involved in $\mathrm{JH}$ synthesis might offer opportunities to identify new targets for alternative control strategies.

Chapter 2 describes the characterization of an A. aegypti corpora allata farnesyl phosphatase, the enzyme responsible for the conversion of farnesyl pyrophosphate (FPP) into farnesol in the biosynthesis of juvenile hormone.

Chapter 3 reports the characterization of an A. aegypti corpora allata mevalonate kinase, the enzyme responsible for the conversion of mevalonate into phosphomevalonate. This chapter describes for the first time the existence of a negative feedback on juvenile hormone synthesis through the action of isoprenoids on the mevalonate kinase activity.

Chapter 4 reports the partial characterization of the activities of recombinant HMG-CoA synthase, phosphomevalonate kinase and FPP synthase from A. aegypti

Chapter 5 summarizes the conclusions of my research and the future directions of studies aiming to complete the characterization of the enzymes of the JH biosynthetic pathway. 


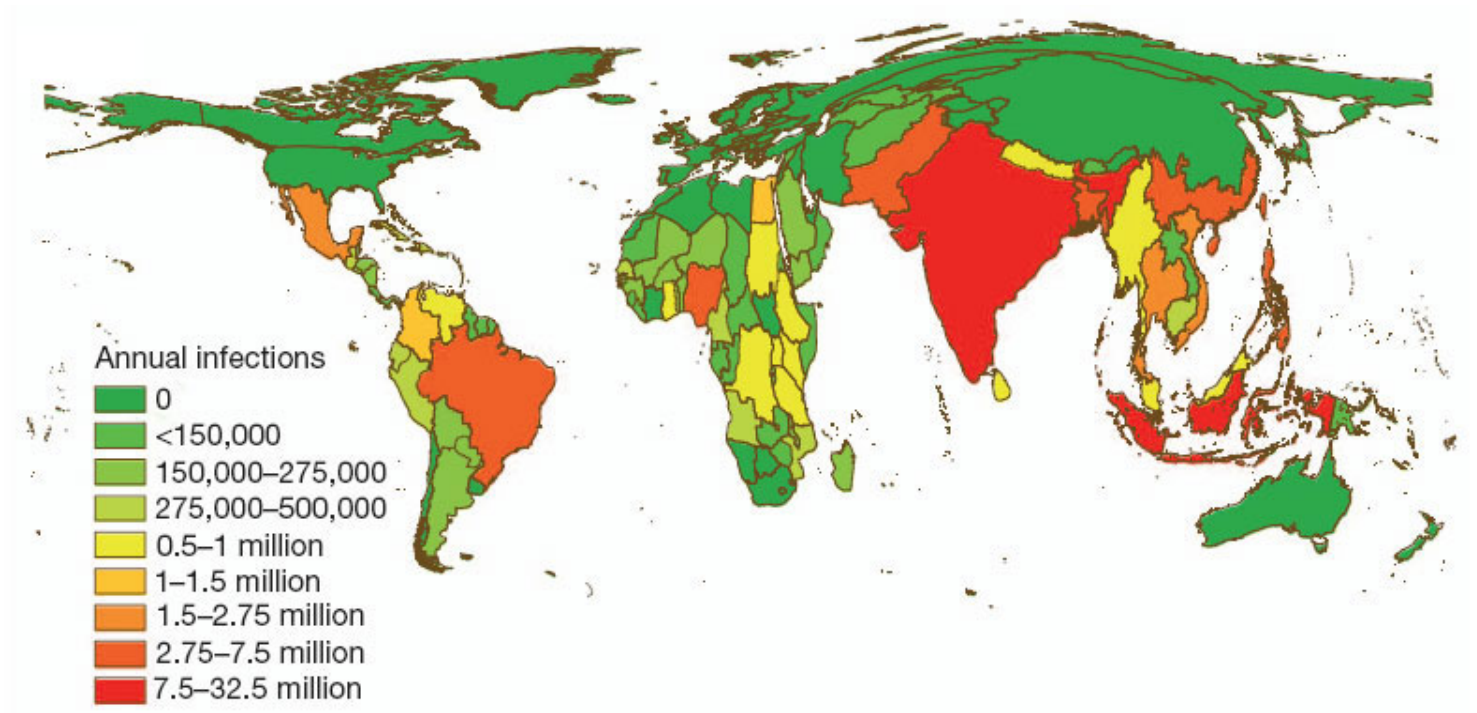

Figure 1: Current global burden of dengue. The map represents the cartogram of the annual number of infections for all ages, as a proportion of national or subnational geographical area (Bhatt et al., 2013). 


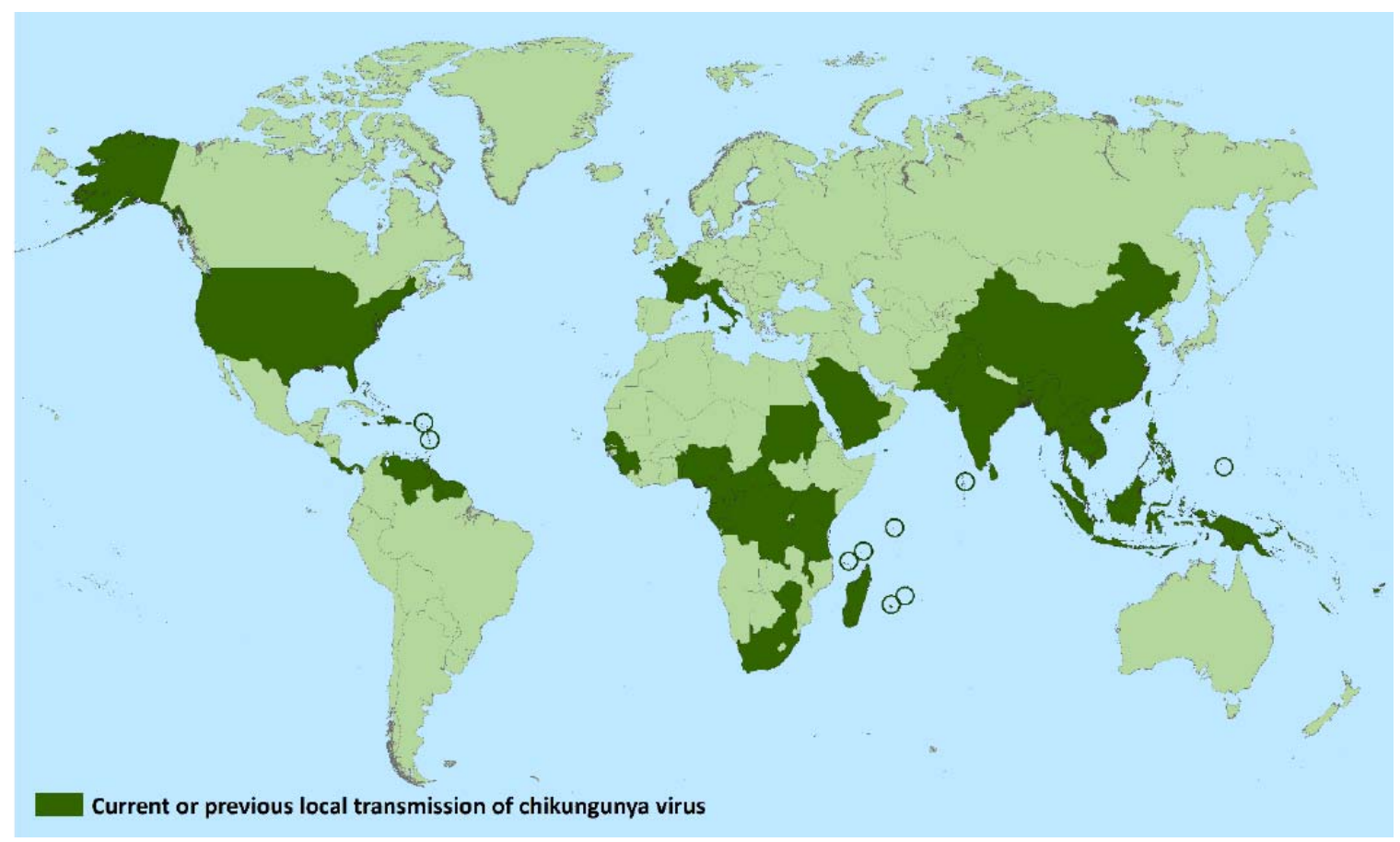

Figure 2: Map of the countries and territories where chikungunya cases have been reported. Dark green represent the areas where chikungunya have been reported (as of August 26, 2014, map courtesy WHO, 2014). 
<smiles>CC/C(=C\CC/C(C)=C/C(=O)OC)CCC1OC1(C)CC</smiles>

JH I<smiles>CCC1(C)OC1CC/C(C)=C/CC/C(C)=C/C(=O)OC</smiles>

JH II<smiles>COC(=O)C=C(C)CCC=C(C)CCC1OC1(C)C</smiles><smiles>CC/C(=C\CC1OC1(C)CC)CC/C=C(\CC)CCC1OC1C(C)=O</smiles>
JHO<smiles>CC(COc1ccc(Oc2ccccc2)cc1)Oc1ccccn1</smiles>

Pyriproxifen<smiles>CC/C(=C\CC(C)/C(=C/C(=O)OC)CC)CCC1OC1(C)CC</smiles>

Iso-JH 0<smiles>COC(=O)C=C(C)CCC1OC1(C)CCC1OC1(C)C</smiles>
JHB3<smiles>COC(=O)C1OC1(C)CCC=C(C)CCC1OC1(C)C</smiles><smiles>CCCC=C(C)CCCC(C)=CC(=O)OC</smiles><smiles>C=C(NCCOc1ccc(Oc2ccccc2)cc1)OCC</smiles>

Fenoxycarb

Figure 3: Structure of juvenile hormones. Chemical strutures of major naturally occuring JH homologues and commonly used JH agonists (from Goodman and Cusson, 2012). 


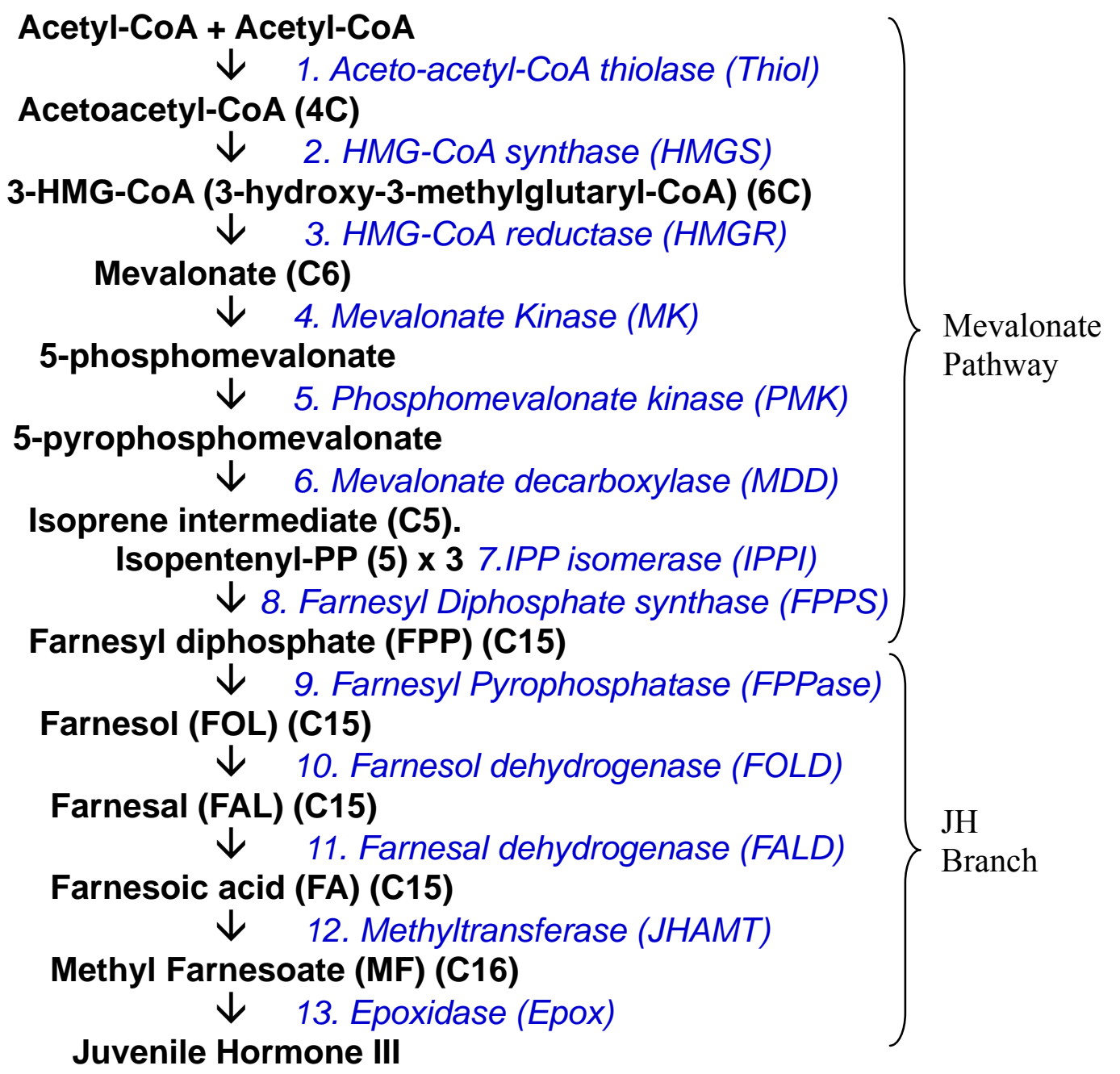

Figure 4: Juvenile hormone III (JH III) biosynthetic pathway in mosquitoes. The biosynthetic pathway of JH III in the CA of mosquitoes is divided into two steps: early step (mevalonate pathways, MVP) and late steps (JH branch of the MVP) (modified from Belles et al., 2005). Precursors are shown in bold and connected by arrows. Enzymes are shown in italic and numbers before the enzymes refer to their positions in the pathway. Abbreviations for the enzymes are between brackets. 

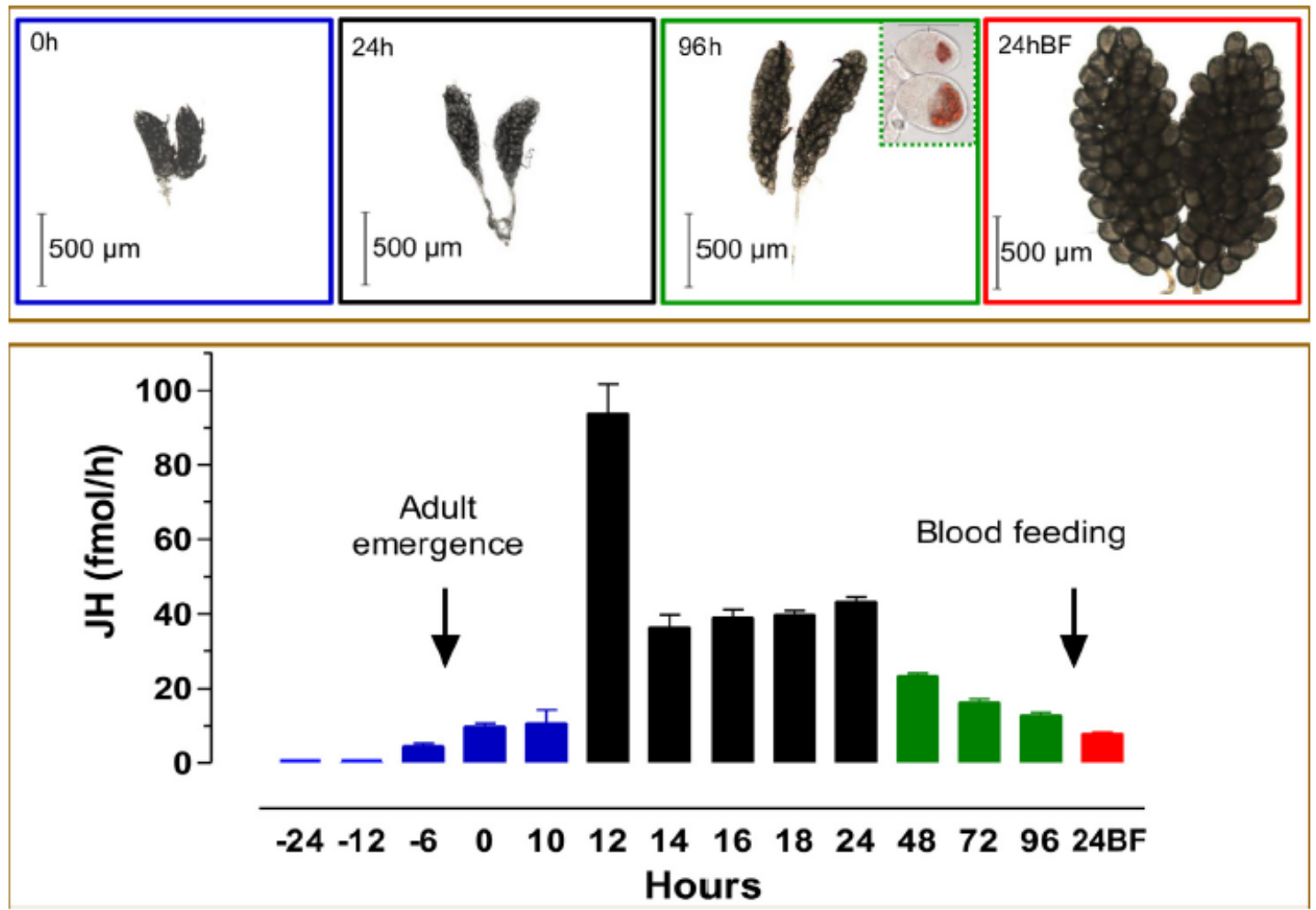

Figure 5: Rate of JH biosynthesis and ovarian development in female Aedes aegypti. Top panel: representative images of the progression of ovary development from emergence to $24 \mathrm{~h}$ after blood feeding. The inset in $96 \mathrm{~h}$ shows the lipid content of follicles from females fed 3\% sugar (top) and 20\% sugar (bottom). Colors for the panels match colors for the CA physiological phases described in the bottom panel. Bottom panel: $\mathrm{JH}$ biosynthesis by CA dissected from pupa, sugar-fed and blood-fed adult females. Hours represent times before (pupa) and after adult emergence (sugar-fed), or after blood feeding (BF). Colors represent the four distinct CA physiological phases identified: inactive or low activity CA (blue), active CA (black), modulated CA (green) and suppressed CA (red) (River-Perez et al., 2014) 


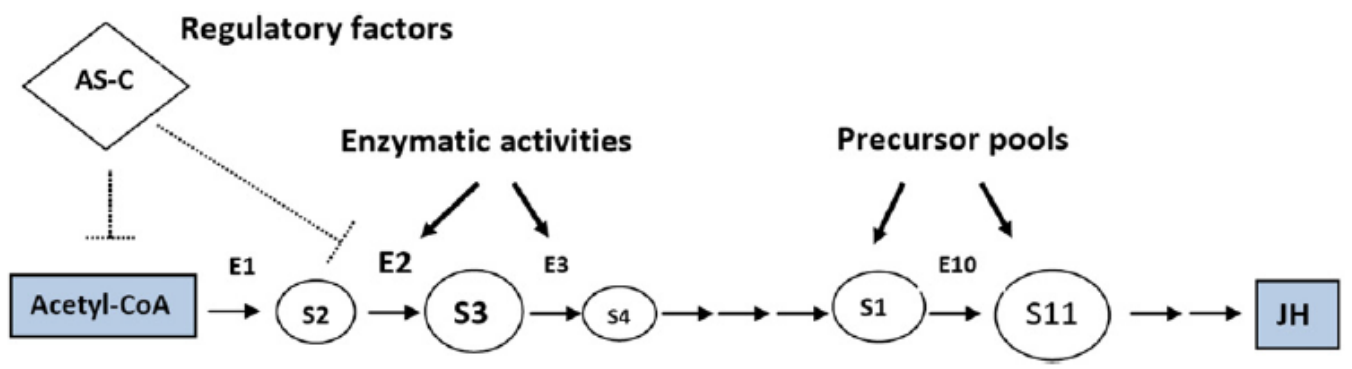

Figure 6: Flux model of juvenile hormone synthesis. This is a schematic representation of a working model for the control of the flux of precursors in the JH biosynthetic pathway. Precursor pools (Acetyl-CoA, S2, etc.) are represented by circles and connected by arrows. E: Enzymes are followed by a number that refers to the position in the pathway (from Nouzova et al., 2011). 


\subsection{References}

1. Adams ME, Kim YJ, Park Y, Zitnan D (2006) Developmental Peptides: ETH, Corazonin, and PTTH In Handbook of Biologically Active Peptides (Abba J. Kastin ed), Burlington: Academic Press, pp. 163-169.

2. Areiza M, Nouzova M, Rivera-Perez C, Noriega FG (2014) Ecdysis triggering hormone ensures proper timing of juvenile hormone biosynthesis in pharate adult mosquitoes. Insect Biochem Mol Biol doi: 10.1016/j.ibmb.2014.09.006.

3. Arik AJ, Rasgon JL, Quicke KM, Riehle MA (2009) Manipulating insulin signaling to enhance mosquito reproduction. BMC Physiol 9:1-11.

4. Attardo GM, Hansen IA, Raikhel AS (2005) Nutritional regulation of vitellogenesis in mosquitoes: implications for anautogeny. Insect Biochem Molec Biol 35:661-675.

5. Barrett ADT, Higgs S (2007) Yellow fever: A disease that has yet to be conquered. Ann Rev 52:209-229.

6. Belgacem YH, Martin JR (2007) Hmgcr in the corpus allatum control sexual dimorphism of locomotor activity and body size via the insulin pathway in Drosophila. PLoS ONE 2(1): e187. doi: 10.1371/journal.pone.0000187

7. Belles X, Martin D, Piulachs MD (2005) The mevalonate pathway and the synthesis of juvenile hormone in insects. Ann Rev Entomol 50:181-199.

8. Bergot BJ, Baker FC, Cerf DC, Jameison G, Schooley DA (1981) Qualitative and quantitative aspects of juvenile hormone titers in developing embryos of several insect species: discovery of new JH like substances discovery from the eggs of Manduca sexta. In Juvenile Hormone Biochemistry (GE Pratt and GT Brooks eds), Amsterdam: Elsevier/North Holland Biomedical Press, pp. 33-45.

9. Bhaskaran G, Sparagana SP, Barrera P, Dahm KH (1986) Change in corpus allatum function during metamorphosis of the tobacco hornworm Manduca sexta: regulation at the terminal step in juvenile hormone biosynthesis Arch Insect Biochem Physiol 276:7465-7474.

10. Bhatt S, Gething PW, Brady OJ, Messina JP, Farlow AW, Moyes CL, Drake JM, Brownstein JS, Hoen AG, Sankoh O, Myers MF, George DB, Jaenisch T, Wint GR, Simmons CP, Scott TW, Farrar JJ, Hay SI (2013) The global distribution and burden of dengue. Nature 496:504-507.

11. Boggs CL (2009) Understanding insect life histories and senescence through a resource allocation lens, Functional Ecology, vol. 23, no. 1, pp. 27-37. 
12. Briegel H 1990 Metabolic relationship between female body size, reserves and fecundity of Aedes aegypti. J Insect Physiol 36:165-172.

13. Brown MR, Clark KD, Gulia M, Zhao Z, Garczynski SF, Crim JW, Suderman RJ, Strand MR (2008) An insulin like peptide regulates egg maturation and metabolism in the mosquito Aedes aegypti, Proc Natl Acad Sci USA 15:5716-21.

14. Buesa C, Martinez-Gonzalez J, Casals N, Haro D, Piulachs MD (1994) Blattella germanica has two HMG-CoA synthase genes. Both are regulated in the ovary during the gonadotrophic cycle J Biol Chem 269:11707-13.

15. Cao L, Zhang P, Grant DF (2009) An insect farnesyl phosphatase homologous to the N-terminal domain of soluble epoxide hydrolase. Biochem Biophys Res Comm 380:188-192.

16. Caroci AS, Li Y, Noriega FG (2004) Reduced juvenile hormone synthesis in mosquitoes with low teneral reserves reduces ovarian previtellogenic development in Aedes aegypti, J Exp Biol 207:2685-2690.

17. Casals N, Buesa C, Piulachs MD, Cabano J, Marrero PF, Belles X, Hegardt FG (1996) Coordinated expression and activity of 3-hydroxy-3-methylglutaryl coenzyme A synthase and reductase in the fat body of Blattella germanica (L.) during vitellogenesis. Insect Biochem Mol Biol 26(8-9):837-43.

18. Clark AJ, Bloch K (1959) The absence of sterol synthesis in insects. J Biol Chem 254:2578-2582.

19. Clifton ME, Noriega FG (2011) Nutrient limitation results in juvenile hormonemediated resorption of previtellogenic ovarian follicles in mosquitoes J Insect Physiol $57: 1274-1281$.

20. Clifton ME, Noriega FG (2012) The fate of follicles after a blood meal is dependent on previtellogenic nutrition and juvenile hormone in Aedes aegypti. J Insect Physiol 58:1007-1019.

21. Cusson M, Sen SE, Shinoda T (2013) Juvenile Hormone Biosynthetic Enzymes as Targets for Insecticide Discovery. In Advanced Technologies for Managing Insect Pests (Ishayya I, Palli SR, Horowitz AR eds), Springer pp. 31-55.

22. Daimon T, Kozaki T, Niwa R Kobayashi I, Furuta K, Namiki T, Uchino K, Banno Y, Katsuma S, Tamura T, Mita K, Sezutsu H, Nakayama M, Itoyama K, Shimada $T$, Shinoda T (2012) Precocious metamorphosis in the juvenile hormone-deficient mutant of the silkworm, Bombyx mori, PLoS Genetics 8(3):e1002486. 
23. Defelipe LA, Dolghih E, Roitberg AE, Nouzova M, Mayoral JG, Noriega FG, Turjanski, AG (2011) Juvenile Hormone Synthesis: "esterify then epoxidize" or "epoxidize then esterify"? Insights from the Structural Characterization of Juvenile Hormone Acid Methyltransferase Insect Biochem Molec Biol 41:228-235.

24. Diaz M, Mayoral JM, Priestap H, Nouzova M, Rivera-Perez C, Noriega FG (2012) Characterization of an isopentenyl diphosphate isomerase involved in the juvenile hormone pathway in Aedes aegypti. Insect Biochem Molec Biol 42:751-757.

25. Dubrovsky EB 2005 Hormonal cross talk in insect development. Trends Endocrinol Metab 16:6-11.

26. Feyereisen R, Farnsworth DE (1987) Characterization and regulation of HMG-CoA reductase during a cycle of juvenile hormone synthesis. Mol Cell Endocrinol 53(3):227-38.

27. Frick S, Nagel R, Schmidt A, Bodemann RR, Rahfeld P, Pauls G, Brandt W, Gershenzon J, Boland W, Burse A (2013) Metal ions control product specificity of isoprenyl diphosphate synthases in the insect terpenoid pathway Proc Natl Acad Sci USA 110(11):4194-9.

28. Gilbert LI, Granger NA, Roe RM (2000) The juvenile hormones: Historical facts and speculations on future research directions. Insect Biochem Mol Biol 30:617-644.

29. Goodman WG, Cusson M (2012) The Juvenile Hormones. In Insect Endocrinology (Gilbert LI, ed), Elsevier, pp. 310-365.

30. Goodman WG, Granger NA (2005) The Juvenile Hormones. In Comprehensive Molecular Insect Science (Gilbert, LI ed) Elsevier, pp. 320-408.

31. Gubler DJ (2002) Epidemic dengue/dengue hemorrhagic fever as a public health, social, and economic problem in the 21st century. Trends Microbiol 10:100-103.

32. Gu SH, Chow YS (1996) Regulation of juvenile hormone biosynthesis by ecdysteroid levels during the early stages of the last two larval instars of Bombyx mori. J. Insect Physiol. 42:625-632.

33. Hales S, de Wet N, Maindonald J, Woodward A (2002) Potential effect of population and climate changes on global distribution of dengue fever: an empirical model. Lancet 360:830-834.

34. Helvig C, Koener JF, Unnithan GC and Feyereisen R (2004) CYP15A1, the cytochrome P450 that catalyzes epoxidation of methyl farnesoate to JH III in cockroach corpora allata Proc Natl Acad Sci USA 101:4024-4029. 
35. Hiruma K, Kaneko Y (2013) Hormonal regulation of insect metamorphosis with special reference to juvenile hormone biosynthesis. Curr Top Dev Biol. 103:73-100.

36. Horodyski FM, Verlinder H, Filkin N, Vandermissen HP, Fleury C, Reynolds SE, Vanden Broeck J (2011) Isolation and functional characterization of an allatotropin receptor from Manduca sexta. Insect Biochem Molec Biol 41:804-814.

37. Howell JJ and Manning BD (2011) mTOR couples cellular nutrient sensing to organismal metabolic homeostasis. Trends Endoc Metab 22, 94-102.

38. Johnson EC, Bohn LM, Barak LS, Birse RT, Nassel DR (2003) Identification of Drosophila neuropeptide receptors by $\mathrm{G}$ protein-coupled receptors- $\beta$-arrestin2 interactions. J. Biol. Chem. 278:52172-78.

39. Judy KJ, Schooley DA, Dunham LL, Hall MS, Bergot J, Siddall JB (1973) Isolation, structure and absolute configuration of a new natural insect juvenile hormone from Manduca sexta. Proc Natl Acad Sci USA 70:1509-1513.

40. Kaneko Y, Kinjoh T, Kiuchi M, Hiruma K (2011) Stage-specific regulation of juvenile hormone biosynthesis by ecdysteroid in Bombyx mori. Mol Cell Endocrinol 335(2):204-10.

41. Klowden MJ 1997 Endocrine aspects of mosquito reproduction. Arch Insect Biochem Physiol 35:491-512.

42. Kotaki T, Shinada T, Kaihara K, Ohfune Y, Numata H (2009) Structure determination of a new juvenile hormone from a heteropteran insect. Org. Lett., 11:5234-5237.

43. Kreienkamp HJ, Larusson HJ, Witte I, Roeder T, Birgül N, Hönck HH, Harder S, Ellinghausen G, Buck F, Richter D (2002) Functional annotation of two orphan Gprotein-coupled receptors, Drostar-1 and -2, from Drosophila melanogaster and their ligands by reverse pharmacology. J Biol Chem 42:39937-39943.

44. Kreienkamp HJ, Liew CW, Bächner D, Mameza MG, Soltau M, Quitsch A, Christenn M, Wente W, Richter D (2004) Physiology of Somatostatin Receptors: from Genetics to Molecular Analysis. In Somatostatin (Srikant CB ed), Kluwer Academic Publishers, Boston, pp. 185-202.

45. Li Y, Unnithan C, Veenstra J, Feyereisen R, Noriega FG 2003b Stimulation of Juvenile hormone biosynthesis by the corpora allata of adult Aedes aegypti in vitro: effect of farnesoic acid and Aedes allatotropin. J Exp Biol 206:1825-1832. 
46. Li YP, Hernandez-Martinez S, Unnithan GC, Feyereisen R, Noriega FG, 2003a Activity of the corpora allata of adult female Aedes aegypti: Effects on mating and feeding. Insect Biochem Mol Biol 33:1307-1315.

47. Lombard J, Moreira D, (2010) Origins and early evolution of the mevalonate pathway of isoprenoid biosynthesis in the three domains of life. Mol Biol Evol 28:87-99.

48. Maestro JL, Cobo J, Belles X (2009) Target of rapamycin (TOR) mediates the transduction of nutritional signals into juvenile hormone production. J Biol Chem 284:5506-5013.

49. Margam, VM., Gelman, DB., Palli, SR. (2006) Ecdysteroid titers and developmental expression of ecdysteroid-regulated genes during metamorphosis of the yellow fever mosquito, Aedes aegypti . J Insect Physiol 52:558-568.

50. Mayoral JG, Nouzova M, Brockhoff A, Goodwin M, Hernandez-Martinez S, Richter D, Meyerhof W and Noriega FG (2010) Allatostatin-C receptors in mosquitoes. Peptides 31: 442-450.

51. Mayoral JG, Nouzova M, Navare A, Noriega FG, (2009a) NADP ${ }^{+}$dependent farnesol dehydrogenase, a corpora allata enzyme involved in juvenile hormone synthesis. Proc Natl Acad Sci USA 106:21091-21096.

52. Mayoral JG, Nouzova M, Yoshiyama M, Shinoda T, Hernandez-Martinez S, Dolghih E, Turjanski AG, Roitberg AE, Priestap H, Perez M, Mackenzie L, Li Y, Noriega FG, (2009b) Molecular and functional characterization of a juvenile hormone acid methyltransferase expressed in the corpora allata of mosquitoes. Insect Biochem Mol Biol 39:31-37.

53. Meyer AS, Schneiderman HA, Hanzmann E, Ko J (1968) The two juvenile hormones from the cecropia silk moth Proc Natl Acad Sci USA 60:853-860.

54. Minakuchi C, Namiki T, Yoshiyama M, Shinoda T (2008) RNAi-mediated knockdown of juvenile hormone acid O-methyltransferase gene causes precocious metamorphosis in the red flour beetle Tribolium castaneum. FEBS J 275:2919-2931.

55. Miziorko HM (2010) Enzymes of the mevalonate pathway of isoprenoid biosynthesis. Arch Biochem Biophys 505:131-143.

56. Morens DM, Fauci AS (2014) Chikungunya at the Door — Deja Vu All Over Again? N Engl J Med doi: 10.1056/NEJMp1408509.

57. Moshitzky P, Applebaum SW (1995) Pathway and regulation of JH III bisepoxide biosynthesis in adult Drosophila melanogaster corpus allatum. Arch Insect Biochem Physiol 30:225-286. 
58. Nijhout HF (1994) Insect Hormones. Princeton, N.J: Princeton University Press.

59. Niwa R, Niimi T, Honda N, Yoshiyama M, Itoyama K, Kataoka H, Shinoda T (2008) Juvenile hormone acid $\mathrm{O}$-methyltransferase in Drosophila melanogaster. Insect Biochem Mol Biol 38:714-720.

60. Noriega FG (2004) Nutritional regulation of JH synthesis: a mechanism to control reproductive maturation in mosquitoes? Insect Biochem Mol Biol 34:687-693.

61. Noriega FG (2014) Juvenile Hormone Biosynthesis in Insects: What Is New, What Do We Know, and What Questions Remain? ISRN 2014:967361.

62. Noriega FG, Ribeiro JMC, Koener JF, Valenzuela JG, Hernandez-Martinez S, Pham VM, Feyereisen R (2006) Comparative genomics of insect juvenile hormone biosynthesis. Insect Biochem Mol Biol 36:366-374.

63. Nouzova M, Edwards MJ, Mayoral JG, Noriega FG, 2011 A coordinated expression of biosynthetic enzyme controls the flux of juvenile hormone precursors in the corpora allata of mosquitoes. Insect Biochem Mol Biol 9:660-669.

64. Nouzova M, Mayoral JM, Brockhoff A, Goodwin M, Meyerhof W, Noriega FG (2012) Functional characterization of an allatotropin receptor expressed in the corpora allata of mosquitoes. Peptides 34:201-208.

65. Nouzova M, Rivera-Perez C, Noriega FG Allatostatin-C reversibly blocks the transport of citrate out of the mitochondria and inhibits juvenile hormone synthesis in mosquitoes. In prep.

66. Nyati P, Nouzova M, Rivera-Perez C, Clifton ME, Mayoral JG Noriega FG (2013) Farnesyl phosphatase, a corpora allata enzyme involved in juvenile hormone synthesis in Aedes aegypti. PLoS ONE 8(8): e71967. doi:10.1371/journal.pone.0071967.

67. Nyati P, Rivera-Perez C, Noriega FG Negative feedback by isoprenoids upon mevalonate kinase activity might regulate juvenile hormone synthesis in Aedes aegypti. In prep

68. Perez-Hedo M, Rivera-Perez C and Noriega FG (2013) The Insulin/TOR signal transduction pathway is involved in the nutritional regulation of juvenile hormone synthesis in Aedes aegypti. Insect Biochem Molec Biol 43:495-500.

69. Perez-Hedo M, Rivera-Perez C, Noriega FG (2014) Starvation increases insulin sensitivity and reduces juvenile hormone synthesis in mosquitoes. PLoS ONE9(1): e86183. doi: 10.1371/journal.pone.0086183. 
70. Piepho H 1938 Wachstum und totale metamorphose an hautimplantaten bei der Wachsmotte Galleria mellonella L. Bio. Zbl. 58:356-366.

71. Quistad GB, Cerf DC, Schooley DA, Staal GB (1981) Fluoromevalonate acts as an inhibitor of juvenile hormone biosynthesis. Nature 289:176-177.

72. Richard DS, Applebaum SW and Gilbert LI (1989) Development regulation of juvenile hormone biosynthesis by the ring gland of Drosophila melanogaster J Comp Physiol 159:383-387.

73. Riehle MA, Brown MR (1999) Insulin stimulates ecdysteroid production through a conserved signaling cascade in the mosquito Aedes aegypti. Insect Biochem Molec Biol. 29:855-860.

74. Riehle MA, Fanb Y, Caoc C, Brown MR (2006) Molecular characterization of insulin-like peptides in the yellow fever mosquito, Aedes aegypti: expression, cellular localization, and phylogeny. Peptides 27:2547-2560.

75. Rivera-Perez C, Nouzova M, Clifton ME, Martin Garcia E, LeBlanc E, Noriega FG (2013) Aldehyde dehydrogenase 3 converts farnesal into farnesoic acid in the corpora allata of mosquitoes. Insect Biochem Mol Biol 43:675-682.

76. Rivera-Perez C, Nouzova M, Lamboglia I, Noriega FG (2014) Metabolic analysis reveals changes in the mevalonate and juvenile hormone synthesis pathways linked to the mosquito reproductive physiology. Insect Biochem Mol Biol 51:1-9.

77. Rivera-Perez C, Nyati P, Noriega FG A corpora allata prenyltransferase in mosquito displaying a metal ion substrate specificity. In prep.

78. Roller H, Dahm KH, Sweeley CC, Trost BM (1967) The structure of the juvenile hormones. Angew Chemie 4:190-191.

79. Schooley DA, Baker FC (1985) Juvenile Hormone Biosynthesis. In Comprehensive Insect Physiology Biochemistry and Pharmacology Kerkut, G.A., (Gilbert LI ed), volume 7, Pergamon Press, Oxford, pp. 363-389.

80. Sen SE, Tomasello A, Grasso M, Denton R, Macor J, Beliveau C, Cusson M, Crowell DN (2012) Cloning, expression and characterization of lepidopteran isopentenyl diphosphate isomerase. Insect Biochem Mol Biol 42:739-750.

81. Shinoda T, Itoyama K (2003) Juvenile hormone acid methyltransferase: a key regulatory enzyme for insect metamorphosis. Proc Natl Acad Sci USA 100:1198611991. 
82. Siddle K, (2012) Molecular basis of signaling specificity of insulin and IGF receptors: neglected corners and recent advances. Front Endoc 3:1-24.

83. Sim C, Denlinger DL (2008). Insulin signaling and FOXO regulate the overwinter diapause of the mosquito Culex pipiens. Proc Natl Acad Sci 105:6777-6781.

84. Smit A, Mushegian A (2000) Biosynthesis of isoprenoids via mevalonate in archaea: the lost pathway. Genome Res 10:1468-1484.

85. Sperry AE, Sen SE (2001) Farnesol oxidation in insects: evidence that the biosynthesis of insect juvenile hormone is mediated by alcohol oxidase. Insect Biochem Mol Biol 31:171-178.

86. Stay B, Tobe SS 2007 The role of allatostatins in juvenile hormone synthesis in insects and crustaceans. Ann Rev Entomol 52:277-299.

87. Stay B, Tobe SS, Bendena WG (1994) Allatostatins: identification, primary structures, functions and distribution In Advances in Insect Physiology, vol. 25, pp. 267-337.

88. Sutherland TD, Feyereisen R (1996) Target of cockroach allatostatin in the pathway of juvenile hormone biosynthesis. Mol Cell Endocrinol 120:115-123.

89. Tan A, Tanaka H, Tamura T, Shiotsuki T (2005) Precocious metamorphosis in transgenic silkworms overexpressing juvenile hormone esterase. Proc Natl Acad Sci USA 102:11751-56.

90. Tobe SS, Stay B (1985) Structure and regulation of the corpus allatum. Adv Insect Physiol 18:305-432.

91. Tu MP, Yin $\mathrm{CH}$, Tatar, M (2005) Mutations in insulin signaling pathways alter juvenile hormone synthesis in Drosophila melanogaster. Gen Comp Endocrinol 142:347-356.

92. Vuerinckx K, Verlinder H, Lindermans M, Vanden Broeck J Huybrechts R (2011) Characterization of an allatotropin-like peptide receptor in the red flour beetle, Tribolium castaneum. Insect Biochem Molec Biol 41:815-822.

93. WHO (2009) Dengue guidelines for diagnosis, treatment, prevention and control. In Ciceri K, Tissot, P, editor. 2009 ed. Geneva: World Health Organization.

94. WHO (2012) Dengue and severe dengue. World Health Organization Monograph Series (Fact sheet $\left.\mathrm{N}^{\circ} 117\right)$. 
95. WHO (2013). WHO recommended insecticides for indoor residual spraying against malaria vectors. World Health Organization.

96. WHO (2014) Chikungunya. World Health Organization Monograph Series (Fact sheet $\left.\mathrm{N}^{\circ} 327\right)$.

97. Wigglesworth VB. 1936. The function of the corpus allatum in the growth and reproduction of Rhodnius prolixus (Hemiptera). QJ Microsc Sci 79:91-121.

98. Williams CM (1956) The juvenile hormone of insects Nature 178:212-213.

99. Yamanaka N, Yamamoto S, Zitnan D, Watanabe K, Kawada T, Satake H, Kaneko Y, Hiruma K, Tanaka Y, Shinoda T, Kataoka H (2008) Neuropeptide receptor transcriptome reveals unidentified neuroendocrine pathways. PLoS One 3(8): e3048. doi: 10.1371/journal.pone.0003048.

100. Zhou G, Flowers M, Friedrich MK, Horton J, Pennington J, Wells MA 2004a Metabolic fate of $\left[{ }^{14} \mathrm{C}\right]$-labeled meal protein amino acids in Aedes aegypti mosquitoes. J Insect Physiol 50:337-349.

101. Zhou G, Pennington J, Wells MA 2004b. Utilization of pre-existing energy stores of female aedes aegypti mosquitoes. Insect Biochem Mol Biol 50:337-349.

102. Zitnan D, Kim YJ, Zitnanova I, Roller L, Adams ME (2007) Complex steroidpeptide-receptor cascade controls insect ecdysis. Gen Comp Endocrinol 153(13):88-96. 


\section{Chapter 2: Farnesyl phosphatase, a corpora allata enzyme involved in juvenile hormone biosynthesis in Aedes aegypti}

\subsection{Abstract}

The only recognized FPP phosphatase (FPPase) expressed in the corpora allata (CA) of an insect was recently described in Drosophila melanogaster (DmFPPase). A search for orthologs of the DmFPPase in Aedes aegypti led to the identification of 3 putative FPPase paralogs expressed in the CA of the mosquito (AaFPPases-1, -2, and -3). AaFPPase- 1 and AaFPase-2 were found to efficiently hydrolyze farnesyl diphosphate (FPP) into farnesol (FOL) and considered as the members of the NagD family of the Class IIA C2 cap-containing haloalkanoic acid dehalogenase (HAD) super family. Using a newly developed assay utilizing fluorescent tags, we demonstrate that AaFPPase activities were different in CA of sugar and blood-fed females. Injection of dsRNAs resulted in a significant reduction of AaFPPase-1 and AaFPPase- 2 mRNAs, but only depletion of AaFPPase-1 caused a significant decrease on JH synthesis. These results suggest that AaFPPase- 1 is involved in the catalysis of FPP into FOL in the CA of A. aegypti.

\subsection{Introduction}

Characterization of corpora allata (CA) enzymes has been hindered by the minute size of the endocrine gland; recently, the first description of an FPP phosphatase (FPPase) expressed in the CA of an insect was described in Drosophila melanogaster (Cao et al., 2009). This enzyme is a member of the haloalkanoic acid dehalogenase (HAD) super family that catalyzes phosphoryl transfer reactions on a remarkably diverse set of substrates and includes enzymes such as: phosphoesterases, ATPases, 
phosphonatases, dehalogenases and sugar phosphomutases (Allen and DunawayMariano, 2004; Arvind et al., 1998). HAD phosphatases employ an aspartate residue as a nucleophile in a magnesium-dependent phosphoaspartyl transferase reaction. The HAD superfamily is represented in the proteomes of organisms from all three super-kingdoms. The highly conserved structural core of the HAD enzymes consists of a $\alpha / \beta$ domain that adopts the topology typical of the Rossmann $\alpha / \beta$ folds housing the catalytic site and can be distinguished from all other Rossmanoid folds by two unique structural motifs: 1) an almost complete $\alpha$-helical turn, named the 'squiggle', and 2) a $\beta$ hairpin turn, termed the ‘flap' (Lahiri et al., 2004; Allen and Dunaway-Mariano, 2009). The catalytic site is thus a composite of the four loops of the core domain and loop 5 of the cap domain. Whereas the core domain orchestrates the core chemistry, the cap domain functions in adapting that chemistry to a specific substrate (Lahiri et al., 2004).

The HAD superfamily can be divided into three generic classes on the basis of existence and location of a cap domain involved in substrate recognition. Class I possesses a small $\alpha$-helical bundle cap between motifs I and II; Class II displays a cap between the second and third motifs; and Class III members present no cap domain (Lahiri et al., 2004). Members of the HAD phosphatase superfamily have four conserved amino acid signature motifs (Koonin and Tatusov, 1994; Arvind et al., 1998; Seifried et al., 2013). These 4 signature motifs are also well conserved in the FPPase described in Drosophila (DmFPPase) (Cao et al., 2009). Bioinformatics searches for orthologs of the DmFPPase in A. aegypti led to the identification of 3 putative FPPase paralogs expressed in the CA of the mosquito (AaFPPase-1, -2, and -3). Recombinant AaFPPase-1 and AaFPPase-2 were found to efficiently hydrolyze FPP into FOL. Different FPPase 
activities were detected in CA extracts from adult female mosquitoes having diverse $\mathrm{JH}$ biosynthetic rates. Injection of dsRNAs resulted in a significant reduction of AaFPPase-1 and AaFPPase-2 mRNAs, but only reduction of AaFPPase-1 caused a significant decrease on JH biosynthesis. These results suggest that AaFPPase- 1 is predominantly involved in the catalysis of FPP into FOL in the CA of A. aegypti.

\subsection{Materials and Methods}

\subsubsection{Chemicals}

Farnesyl diphosphate (FPP), geranyl diphosphate (GPP) and isopentenyl diphosphate (IPP) were purchased from Echelon Biosciences (Salt Lake City, UT). We purchase p-nitrophenyl phosphate from MP Bio medicals (Santa Ana, CA). The N-acetylS-geranylgeranyl-L-cysteine (AGGC) and N-acetyl-S-farnesyl-L-cysteine (AFC) were purchased from Cayman chemicals (Ann Arbor, MI). Taurolithocholic acid 3-sulfate was purchased from Sigma-Aldrich (St. Louis, MO).

\subsubsection{Insects}

Aedes aegypti of the Rockefeller strain were reared at $28^{\circ} \mathrm{C}$ and $80 \%$ relative humidity under a photoperiod of $16 \mathrm{~h}$ light: $8 \mathrm{~h}$ dark. A cotton pad soaked in $3 \%$ sucrose solution was provided to adults. Four-day-old female mosquitoes were membrane-fed porcine blood equilibrated to $37^{\circ} \mathrm{C}$, and ATP was added to the blood meal to a final concentration of $1 \mathrm{mM}$ immediately before use.

\subsubsection{Expression of recombinant AaFPPases}

The AaFPPase cDNAs were expressed in E. coli cells as described by Mayoral et al., 2009. Recombinant His-tagged proteins were purified using HiTrap chelating columns and PD-10 desalting columns (Amersham Pharmacia, Piscataway, NJ). Glycerol 
was added to the enzyme solution (final concentration 50\%), and samples were stored at $20^{\circ} \mathrm{C}$ until used. Protein concentrations were determined using the bicinchoninic acid protein assay reagent (BCA) (Pierce, Rockford, IL). Bovine serum albumin was used as a standard.

\subsubsection{Enzyme assays}

\subsubsection{Phosphatase assay}

The catalytic activity of recombinant AaFPPases towards $p$-NPP was measured in 96 well plates as described by Cao et al., 2009. Phosphatase activities towards different isoprenoid pyrophosphate substrates were determined using the Malachite Green Phosphate Assay Kit (Bioassay Systems, Hayward, CA); enzymatic activities were assayed using $40 \mu \mathrm{L}$ reaction mixtures containing $100 \mathrm{mM}$ MES, $\mathrm{pH}$ 6.0, $2 \mathrm{mM} \mathrm{MgCl}$, substrate $(150 \mu \mathrm{M})$ and $75 \mathrm{ng}$ of enzyme. After $20 \mathrm{~min}$ of incubation at $37^{\circ} \mathrm{C}$, the reaction was terminated by the addition of the malachite green reagent $(4: 1 \mathrm{v} / \mathrm{v})$, and $30 \mathrm{~min}$ later the production of $\mathrm{Pi}$ was measured at $630 \mathrm{~nm}$ using a BioTek plate reader (BioTek, Winooski, VT). Kinetic parameters were determined by non-linear curve fitting using the GraphPad Prism software (San Diego, CA).

\subsubsection{RP-HPLC analysis of FPPase catalytic products.}

Production of FOL from FPP hydrolysis was analyzed by reverse-phase HPLC. FPP $(250 \mu \mathrm{M})$ was incubated with recombinant AaFPPase for $60 \mathrm{~min}$ in buffer $(100 \mathrm{mM}$ MES, pH 6.0, $2 \mathrm{mM} \mathrm{MgCl2).} \mathrm{Reactions} \mathrm{were} \mathrm{terminated} \mathrm{by} \mathrm{adding} 500 \mu 1$ of acetonitrile. Samples were centrifuged at 14,000 rpm for $5 \mathrm{~min}$ and the organic phase was recovered, filtered and analyzed by reverse-phase HPLC on a Dionex Summit System (Dionex, Sunnyvale, CA) equipped with a UVD $170 \mathrm{U}$ detector, 680 HPLC pump, TCC 100 
column oven and Chromeleon software. The HPLC analysis was performed on an analytical column Acclaim 120 C18 (250 X 2.1 mm ID, particle size $5 \mu \mathrm{m})$ (Dionex), using isocratic elution from 0 to $20 \mathrm{~min}$ (acetonitrile-water, 1:1 v/v), followed by a linear gradient from 20 to $50 \mathrm{~min}$ (acetonitrile-water (50 to $95 \%, \mathrm{v} / \mathrm{v}$ ) and another isocratic elution from $50 \mathrm{~min}$ (acetonitrile, 95\%). Flow rate was $0.2 \mathrm{ml} / \mathrm{min}$ and column temperature was $25^{\circ} \mathrm{C}$. The eluate was monitored with UV (214 nm). Water or/and glycerol were used in place of recombinant enzymes in negative controls.

\subsubsection{Effect of inhibitors on AaFPPase activity}

Recombinant AaFPPases were pre-incubated with different concentrations (0 to $40 \mu \mathrm{M})$ of putative inhibitors for 10 min and their activities were measured using the $p$ NPP assay. The following compounds were tested: N-acetyl-S-geranylgeranyl-L-cysteine (AGGC), N-acetyl-S-farnesyl-L-cysteine (AFC) and taurolithocholic acid 3-sulfate.

\subsubsection{Quantitative real-time PCR (qPCR)}

The RNA isolation and qPCR were performed as described by Nouzova et al., 2011. The primers and probes for the house keeping gene $60 \mathrm{~S}$ ribosomal protein rpL32 and AaFPPase transcripts are included in Table 1.

\subsubsection{RNAi experiments}

Synthesis and microinjections of double-stranded RNA (dsRNA) were performed as described by Perez-Hedo et al., 2014. The AaFPPases and YFP (yellow fluorescent protein) target sequences for dsRNA synthesis were amplified by PCR using the AaFPPase-i and YFP-i primers (Table 1). The resulting amplicons were diluted 50-fold, and $1 \mu \mathrm{l}$ was used as template in PCR reactions with primers containing $\mathrm{T} 7$ promoter sequences (Table 1). The products from these PCR reactions were purified using a 
QIAquick PCR purification kit (QIAquick sciences, Germantown, MD), and 1-2 $\mu \mathrm{g}$ of the purified DNA templates were used to synthesize dsRNAs with a Megascript RNAi kit (Ambion, Austin, TX). dsRNAs were precipitated using ammonium acetate/ethanol, and resuspended in $\mathrm{ddH} 2 \mathrm{O}$ to a final concentration of 3-4 $\mu \mathrm{g} / \mu \mathrm{l}$. In each knockdown experiment, newly emerged female mosquitoes were cold anesthetized and injected intrathoracically with $1.6 \mu \mathrm{g}$ of dsRNA using a Drummond Nanoject II microinjector and a micromanipulator. The effect of dsRNA was evaluated 4 days after injection, a time selected based on the analysis of dsRNA depletion experiments.

\subsubsection{FPPase activity in CA extracts}

FPPase activities in mosquito CA-CC (corpora allata-corpora cardiaca complex) were measured by HPLC coupled to a fluorescent detector (HPLC-FD) monitoring the production of farnesol. Glands were dissected in buffer solution (100 mM MES pH 6.0, 2 $\mathrm{mM} \mathrm{MgCl2}$ ). CA-CC were homogenized for $1 \mathrm{~min}$, sonicated $3 \mathrm{~min}$ and centrifuged at $10,000 \mathrm{~g}$ for $10 \mathrm{~min}$ at $4^{\circ} \mathrm{C}$. Supernatants were recovered and used as crude extract for activity assays as previously described (Rivera-Perez et al., 2013). The reaction products were labeled with DBD-COC1 for further quantification on HPLC-FD (Rivera-Perez et al., 2012). Controls such as boiled crude extract and reactions without enzyme were included. A standard curve was constructed for the quantification of tagged farnesol.

\subsubsection{JH biosynthesis assay}

The amount of JH synthesized by CA-CC complexes in vitro was quantified by high performance liquid chromatography coupled to a fluorescent detector (HPLC-FD) (Rivera-Perez et al., 2012). The assay uses derivatization of JH III with a fluorescent tag with subsequent analysis by reverse phase HPLC-FD. 


\subsubsection{Secondary structure and phylogenetic analysis}

The secondary structure for AaFPPase-1 was predicted using the protein structure homology-modeling server Swiss v.8.05 (Schwede et al., 2003; Arnold et al., 2006) and the Human pyridoxal phosphate phosphatase (2oycA), that share a similarity of $29 \%$, as template. A Maximum-Likelihood tree was built using MEGA software version 5.1 (Tamura et al., 2011), with a bootstrapping of 1000. Pairwise deletion method was selected for the gap/missing data.

\subsubsection{Statistical analysis}

Statistical analyses were performed using the GraphPad Prism Software (San Diego, CA, USA). The results are expressed as means \pm S.E.M. Significant differences (P $<0.05)$ were determined with a one-tailed student t-test or one-way ANOVA followed by a pair-wise comparison of means (Tukey's test).

\subsection{Results}

\subsubsection{Identification of three A. aegypti FPPases expressed in the CA}

Using the sequence of a D. melanogaster FPPase (CG15739) that converts FPP into FOL (DmFPPase) (Cao et al., 2009) we screened the A. aegypti genome (Vectorbase) (Lawson et al., 2009). Eight HAD genes displaying over $48 \%$ amino acid sequence similarity were identified (Genbank accession numbers: AAEL012292, AAEL010099, AAEL010098, AAEL007097, AAEL007094, AAEL007098, AAEL007090 and AAEL009503). By examining the temporal and tissue dependent expression of the 8 HAD genes by PCR we identified 3 HADs that were expressed in the CA of adult female mosquito at appropriate times (Genbank: AAEL010099, AAEL007090 and AAEL009503) (Fig. 7); we named them AaFPPase-1, AaFPPase-2 and 
AaFPPase- 3 respectively, and were further considered as putative AaFPPases that could be involved in JH biosynthesis. Amino acid sequence alignments of $A$. aegypti and $D$. melanogaster FPPases revealed a number of well conserved residues typical of the HAD phosphatases, including an aspartic acid $\left(\mathrm{Asp}^{36}\right)$ that acts as the catalytic nucleophile, a serine or threonine $\left(\mathrm{Ser}^{67}\right)$ for binding the phosphate group and two aspartic acid residues $\left(\mathrm{Asp}^{253}, \mathrm{Asp}^{258}\right.$ ) important for binding the $\mathrm{Mg}^{2+}$ cofactor (Cronin et al., 2003; Seifried et al., 2013) (Fig. 8). The AaFPPase-1 structure obtained by homology modeling exhibited the typical HAD core and cap regions, with the catalytic site as a composite of the four conserved loops of the core region and the loop 5 of the cap region (cap 2 domain) (Fig. $8)$.

A phylogram was generated using FPPase orthologs found in insects and human (Fig. 9). The HAD classes IA and IIA clearly separated in two distinct clusters; the main cluster comprises members of the NagD family included in the class IIA with a C2 cap domain (motif V or loop 5) located between the second and third motif. Each of these amino acid sequences contains the conserved four loops (Motif I-IV). Most of the insects phosphatases identified presented one functional HAD domain in the N-terminal of the protein; with many displaying a second incomplete HAD domain in the C-terminus. In addition, three $D$. melanogaster phosphatases had a second functional HAD domain on the C-terminal. We also identified three D. melanogaster sequences with a single catalytic HAD domain in the C-terminus of the proteins. Two Human HAD phosphatases (phosphoglycolate phosphatase and pyridoxal phosphatase) were also grouped in the class IIA. Finally, as outgroup we used the bi-functional human epoxy hydrolase that belongs to the Class IA, having a $\mathrm{C} 1$ cap located between the motif I and II; this enzyme 
possess both phosphatase and epoxy hydrolase functional domains. We identified three $A$. aegypti orthologs of the epoxy hydrolase, but they only possess the epoxy hydrolase domain.

\subsubsection{All AaFPPases hydrolyzed p-NPP, but only AaFPPase-1 and -2 converted FPP into farnesol}

The three putative AaFPPases were overexpressed in E. coli. Recombinant Histagged proteins $(\sim 35 \mathrm{kDa})$ were purified and phosphatase activities were measured using para-nitrophenyl phosphate (p-NPP), a chromogenic substrate for most phosphatases, including alkaline, acid, protein tyrosine and serine/ threonine phosphatases. AaFPPase-2 $\left(K_{m}=315.5 \pm 46.9 \mu \mathrm{M}\right)$ had higher affinity for $p$-NPP than AaFPPase-1 $\left(K_{m}=3959.43 \pm\right.$ 126.78 $\mu \mathrm{M})$. All AaFPPases increased their catalytic activities in a dose-response manner when $\mathrm{Mg}^{2+}$ was used as a cofactor (Fig. 10) reaching their maximum activity at $\mathrm{pH} 6.0$ (Fig. 10), which is consistent with previous findings in fruit flies (Cao et al., 2009).

The specific activities of AaFPPases toward isoprenoid phosphates were measured using the malachite green assay, in which the amount of released inorganic phosphate is determined by quantifying the formation of a complex between malachite green molybdate and free orthophosphate that absorbs at 620-640 nm (Veldhoven and Mannaerts, 1987). Only AaFPPase-1 and AaFPPase-2 efficiently hydrolyzed FPP into FOL $\left(K_{m}=\sim 222 \mu \mathrm{M}\right)($ Table 2$)$. AaFPPase-1 $\left(K_{m}=184.45 \pm 14.16 \mu \mathrm{M}\right)$ and AaFPPase-2 $\left(K_{m}=273.98 \pm 2.52 \mu \mathrm{M}\right)$ also efficiently hydrolyzed GPP. Both enzymes also demonstrated a low affinity for IPP (Table 1). Both enzymes displayed higher "catalytic efficiencies" for GPP than for FPP with $K_{\text {cat }} / K_{m}$ specificity constants for GPP 3-4 fold higher than those for FPP (Table 2). Conversion of FPP into FOL by AaFPPase-1 and 
AaFPPase-2 was confirmed by RP-HPLC analysis (Fig. 11). For the substrates used in the present study we found no evidence that pyrophosphate was released from AaFPPases catalyzed reactions. The malachite green phosphate assay does not detect pyrophosphate, but only identifies free phosphate released in solution. In addition, when we treated the products of the AaFPPases catalyzed reaction with pyrophosphatase (an enzyme which cleaves a pyrophosphate into two phosphate ions) we did not detect any significant increase in the amount of free phosphate.

Two isoprenoid-derived compounds, AGGC, AFC and a lipid sulfate were evaluated as potential inhibitors of the AaFPPase catalytic activity. While AGGC was a potent inhibitor of AaFPPase-1 and AaFPPase-2 (Fig. 12), AFC and taurolithocholic acid 3-sulfate had little effect.

\subsubsection{The CA exhibited variable FPPase activity}

Corpora allata extracts were able to convert FPP into FOL, with the FPPase catalytic activity increasing more than 4 fold when $2 \mathrm{mM} \mathrm{MgCl} 2$ was added (Fig. 13A). AaFPPase activities were measured in CA extracts from adult female mosquitoes having three distinct $\mathrm{JH}$ biosynthetic conditions: basal activity ( $0 \mathrm{~h}$ or newly emerged adult), high activity ( $24 \mathrm{~h}$ sugar-fed) and suppressed activity ( $24 \mathrm{~h}$ after blood feeding). In the presence of an excess of FPP, highly active glands produced $92 \mathrm{fmol}$ of FOL/CA/h, while suppressed glands produced only $45 \mathrm{fmol}$ of $\mathrm{FOL} / \mathrm{CA} / \mathrm{h}$. The $\mathrm{CA}$ with basal activity from newly emerged females, that produced only $12 \mathrm{fmol} / \mathrm{h}$ of JH, had quite elevated FPPase activity (210 fmol of FOL/CA/h) (Fig. 13B). 


\subsubsection{Tissue- and developmental-stage-specific expression of AaFPPases}

Quantitative real time PCR was used to analyze the tissue- and developmentalstage-specific expression of AaFPPases. All three AaFPPase genes were expressed in the CA, but highest transcript levels were detected in other mosquito tissues. The highest level of AaFPPase-1 mRNA was detected in midgut and Malphigian tubules, while that of AaFPPase-2 mRNA in Malpighian tubules and AaFPPase-3 transcripts were most abundant in brain and ovaries (Fig. 14). A developmental time course of mRNA expression in the CA showed that transcripts of AaFPPase- 1 and AaFPPase-2 were low in late pupae, increased after emergence and peaked at day one in sugar-fed mosquitoes (Fig. 15A). The AaFPPase-3 transcripts levels remained relatively constant for the same period. Transcript levels for the three AaFPPase genes moderately increased after bloodfeeding (Fig. 15B).

\subsubsection{Reduction of AaFPPase-1 by RNAi caused a significant decrease on JH biosynthesis}

Since AaFPPase-3 did not appear to catalyze FPP, it was not further considered to have a major role in $\mathrm{JH}$ biosynthesis. Therefore the effect of mRNA depletion using RNAi was only studied with AaFPPase-1 and AaFPPase-2. Injection of dsRNA resulted in a significant reduction of AaFPPase-1 and AaFPPase-2 mRNAs ( 80\%) (Fig. 16A). Reduction of AaFPPase-1 transcripts resulted in a significant reduction in JH biosynthesis when compared with CA of females treated with dsYFP or dsAaFPPase- 2 (Fig. 16B). 


\subsection{Discussion}

\subsubsection{Molecular and functional characterization of AaFPPases expressed in the corpora allata of mosquitoes}

In this study we identified and characterized two corpora allata mosquito NagD phosphatases that are able to convert FPP into FOL. The homology model of AaFPPase-1 exhibited the typical HAD core and cap regions [Burroughs et al., 2006; Seifried et al., 2013). The core region is considered to be a modular phosphoryl-transfer unit with the squiggle and flap motifs providing a solvent exclusion mechanism that allows HAD enzymes to alternate between "open" and "closed" conformations. The enzyme in the "open' configuration allows the substrate to enter the active site and interact with the highly conserved catalytic residues in the four core motifs and the cap (Arvind et al., 1998; Seifried et al., 2013). Upon cap closure, some residues in the cap domain enter the active site and engage in catalysis. Once the substrate is bound, the enzyme assumes a "closed" configuration and the $\mathrm{Mg}^{2+}$ ion in the active site interacts with the negatively charged phosphate, preparing it for nucleophilic attack by the first conserved aspartate on motif I (Seifried et al., 2013) (Fig. 17). The AaFPPase-1 and previously described DmFPPase (GC15739) (Cao et al., 2009) are both expressed in the CA, process FPP into FOL and are part of a cluster of NagD family members that contain one functional active site (HAD domain) in the N-terminus of the core unit. Additional close related NagD sequences in other insects exhibited variability on the number and location of the HAD domains; although the effect of these changes on activity and substrate specificity remains to be studied. The study of FPPases from additional insect species could help to 
improve our understanding of the basis of isoprenoid phosphate binding specificity in NagD insect proteins.

\subsubsection{Expression of AaFPPases genes}

Previous studies in Bombyx mori (Kinjoh et al., 2007; Ueda et al., 2009) and A. aegypti (Nouzova et al., 2011) suggested that the transcripts for most of the JH biosynthetic enzymes were highly enriched or exclusively expressed in the CA. The last two metabolic reactions, the methylation of FA and the epoxidation of MF, are most likely exclusive for $\mathrm{JH}$ biosynthesis and therefore the enzymes involved (juvenile hormone acid methyl transferase and epoxidase) should be highly expressed in the CA (Nouzova et al., 2011). In contrast, other enzymes in the late pathway, such as the AaFPPases described in these studies, farnesol dehydrogenases (Mayoral et al., 2009) and farnesal dehydrogenases (Rivera-Perez et al., 2013) are broadly expressed in many tissues, which is not surprising since farnesol and farnesal homeostasis are vital for cells in all insect tissues. Farnesol acts as a signaling molecule in cell proliferation and apoptosis (Roullet et al., 1999; Joo and Jetten, 2010; Joune et al., 2008). Posttranslational modifications by attachment of a farnesyl group to C-terminal cysteine of target proteins by farnesyl-transferases are essential for signal transduction and vesicular transport (Pechlivanis and Kuhlmann, 2006). Farnesal dehydrogenases play key roles in the generation of fatty alcohols and fatty acids as well as in the elimination of toxic biogenic and xenobiotic aldehydes, such as those produced by oxidative damage of glycerolipids or during protein deprenylation (Jakoby and Ziegler, 1990; Rizzo and Craft, 1991; Tschantz et al., 2001). The presence of more than one isozyme capable of catalyzing the hydrolysis of long chain pyrophosphates in mosquitoes suggests that selection 
mechanism caused duplication and diversification of members of the NagD family and facilitated the evolution of more efficient substrate specificities, as well as a better tissue and developmental regulation; essential for the critical role that these phosphatases play in every cell.

\subsubsection{AaFPPase-1 and JH biosynthesis}

Using an HPLC-fluorescence approach, we were able to measure the changes in the production of FOL by AaFPPase from CA extracts dissected from newly emerged mosquitoes, sugar-fed and blood-fed female mosquitoes. As was shown with the recombinant proteins, the FPPase activity of the CA extracts were $\mathrm{Mg}^{2+}$ dependent, and exhibited remarkable differences among basal, highly active and depressed glands. In sugar-fed females, we found a good concordance between AaFPPase-1 and -2 mRNA expressions in the CA and JH biosynthesis (Li et al., 2003). Although the highest transcript levels of AaFPPases were found in highly active glands, the maximum enzyme activity was found in basal active glands, suggesting that the molecular basis for $\mathrm{JH}$ regulation is quite unique at different times during the reproductive cycle of an adult female mosquito.

We have previously described a 1000-fold difference in the levels of mRNA expression in the CA among the JH biosynthetic enzymes (Nouzova et al., 2011). Four enzymes presented overall low levels of expression, acetoacetyl-CoA thiolase, phosphomevalonate kinase, farnesol dehydrogenase and farnesal dehydrogenase (Nouzova et al., 2011; Rivera-Perez et al., 2013); transcripts numbers for AaFPPase-1 are also low and comparable to the levels of those 4 genes. Under some conditions any of these enzymes could become rate limiting or "bottleneck". We have reported that the 
low enzymatic activity of farnesal dehydrogenase could be a restrictive factor for $\mathrm{JH}$ biosynthesis in the CA of blood-fed mosquitoes (Rivera-Perez et al., 2013); a similar condition might apply to AaFPPase-1, the decrease in enzymatic activity detected after blood-feeding might reduce the farnesol pool to levels that could limit the flux of precursors and JH biosynthesis.

AaFPPase-1 and -2 efficiently hydrolyzed FPP into FOL. Therefore, we selected these 2 genes for RNAi studies. Although the RNAi mediated silencing was efficient for both enzymes, we found JH biosynthesis was significantly reduced only in AaFPPase- 1 silenced mosquitoes CA, suggesting that AaFPPase-1 is predominantly involved in $\mathrm{JH}$ biosynthesis.

\subsection{Conclusions}

A search for orthologs of a farnesyl phosphatase described in D. melanogaster led to the identification of two NagD AaFPPases that are expressed in the CA of A. aegypti and efficiently hydrolyzed FPP into FOL. A combination of RNAi experiments and biochemical studies using CA extracts and recombinant proteins support the hypothesis that these HAD enzymes convert FPP into FOL in the CA and might be involved in JH biosynthesis in mosquitoes. 


\title{
Primers used for RT-PCR
}

\begin{tabular}{|c|c|c|}
\hline $\begin{array}{l}\text { Gene accession } \\
\text { number }\end{array}$ & Forward Primer & Reverse Primer \\
\hline AAEL012292 & 5'CGTTGATTCGTTCGATTGTG 3' & 5'CGAATGTACGAACGCTGTTG 3' \\
\hline AAEL010099 & 5'GAGGACGTCGTTCATCCAGT 3' & 5'AATCTACCACCACCGCTTTG 3' \\
\hline AAEL010098 & 5’TTCGAGGGTTTGATCTACGC 3' & 5'GAATGCACTCGGGATCACTT 3' \\
\hline AAEL007097 & 5' TCTCCGAAACGAGCAGTACA 3' & 5' GGATTCCGTTCCAGATAGCA 3' \\
\hline AAEL007094 & 5' GTCGTGAGGCTTTGGTTCTC 3' & 5'AATTCTTCGGGCTTGTTGTG 3' \\
\hline AAEL007098 & 5' GTCGTGAGGCTTTGGTTCTC 3' & 5' AATTCTTCGGGCTTGTTGTG 3' \\
\hline AAEL007090 & 5' TTGGGACGGAGGTGTTTAAG 3' & 5' GGCTTTCATGAGATGGGACA 3' \\
\hline AAEL009503 & 5' CATCGAGAATGGGAAGCAGT3' & 5' GGAACCGACCACGTACACTT 3 \\
\hline
\end{tabular}

\section{Primers used for Q-RT-PCR}

Primer

rpL32 Forward

rpL32 Reverse

rpL32 Probe

AaFPPase 1 Forward

AaFPPase 1 Reverse

AaFPPase 1 Probe

AaFPPase 2 Forward

AaFPPase 2 Reverse

AaFPPase 2 Probe

AaFPPase 3 Forward

AaFPPase 3 Reverse

AaFPPase 3 Probe
Sequence

5' CCATCAGTCCGATCGCTATGA 3'

5' GTTGTCAATACCTTTCGGCTTACG 3'

5' CAAGCTTGCCCCCAACTG 3'

5' AGGGATGCAGGGTTTGAaGTTATTC 3'

5' GATAAGACGGAGCGATTCTGGTT 3'

5' ATGGGCCGAACGATGCA 3'

5' GGAGGTGTTTAAGAACTATCTACGATCA 3'

5' GGCGGCGCCTCCAT 3'

5' CCGTTGGCCCATCTAG 3'

5' GTGGCCAAATCGGTGAAACTG 3'

5' GCAGCTAGATAGGCGGTAGAGATAA 3'

5' TTGTCAACGCCAACATT 3'

\section{Primers used for production of dsRNA}

\author{
Primers Sequence \\ AaFPPase-1 5' TAATACGACTCACTATAGGGAGTTATTCATGGGCCGAACGATGC 3' \\ Forward T7 \\ AaFPPase-1 \\ Reverse T7 \\ AaFPPase-2 \\ Forward T7 \\ AaFPPase-2 \\ Reverse T7 \\ YFP-Forward 5' TAATACGACTCACTATAGGGAACCGCATCGAGCTGA 3' \\ $\mathrm{T} 7$ \\ YFP-Reverse 5' TAATACGACTCACTATAGGGATGGTCAGGCGGGACT 3' \\ T7 \\ 5’TAATACGACTCACTATAGGGATATCGACCCGAGCCCAGAACTTCAA3' \\ 5' TAATACGACTCACTATAGgGCGATCAGAAGGATTTACTGTTCTAG 3' \\ 5' TAATACGACTCACTATAGGGCAGCAAACAGTCAGGATTGC 3'
}

Table 1: Primers used for RT-PCR, Q-RT-PCR, and production of dsRNA 


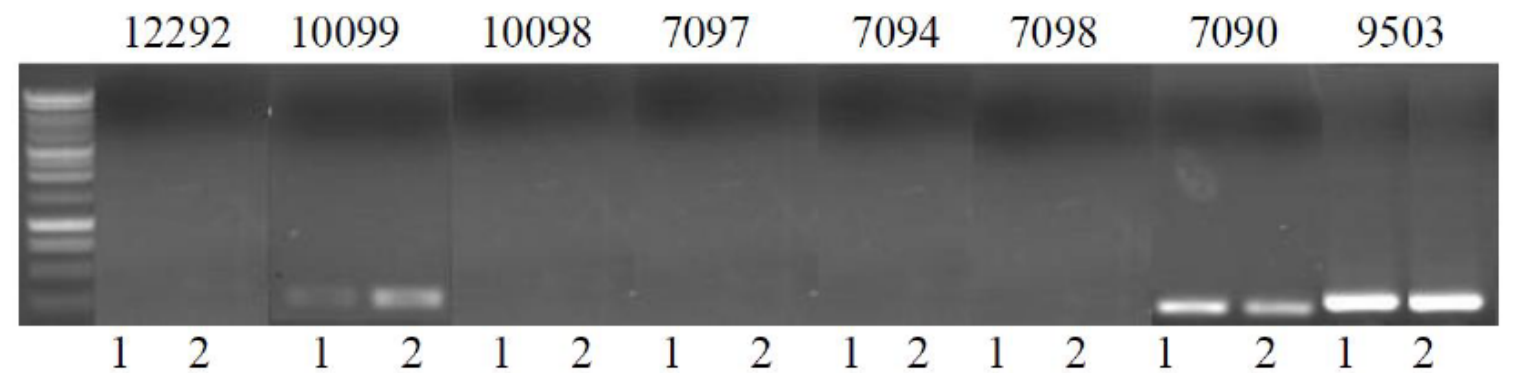

Figure 7: PCR analysis of the expression of eight putative phosphatase genes in the CA of adult female Aedes aegypti. cDNA was made from: (1) CA dissected at the time of adult emergence, (2) one day-old sugar-fed females. From left to right: AAEL012292, AAEL010099 (AaFPPase-1), AAEL010098, AAEL007097, AAEL007094, AEL007098, AAEL007090 (AaFPPase-2) and AAEL009503 (AaFPPase-3). 


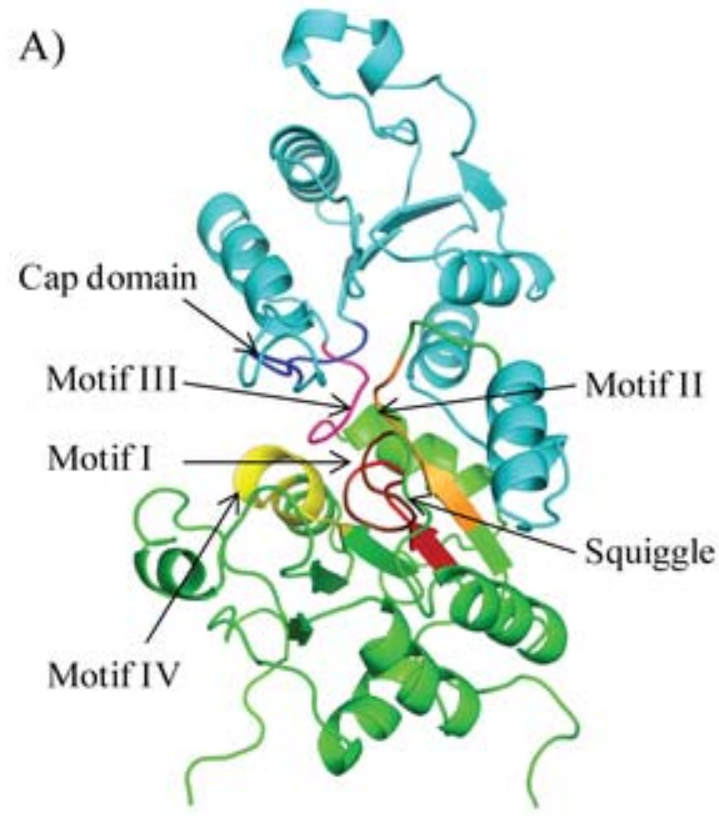

B)

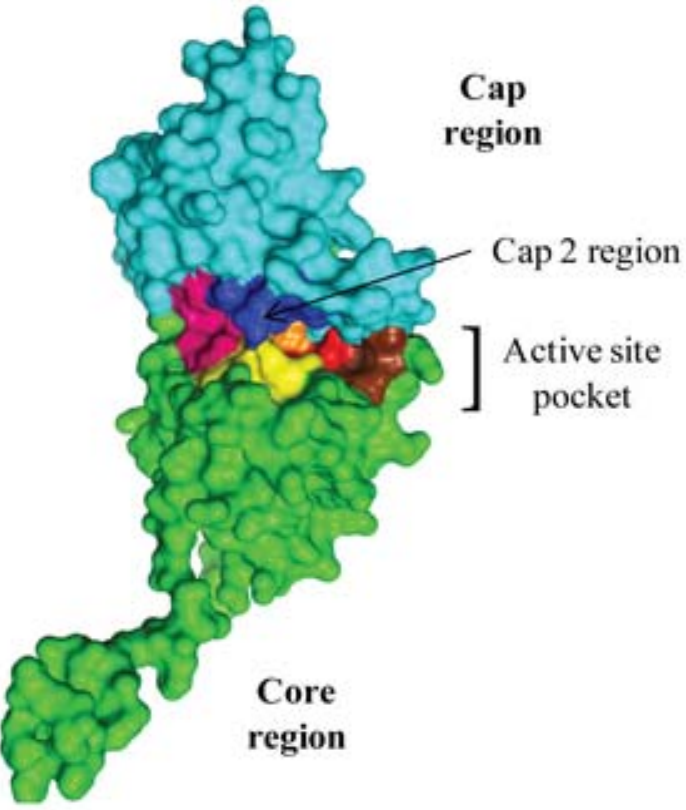

C)

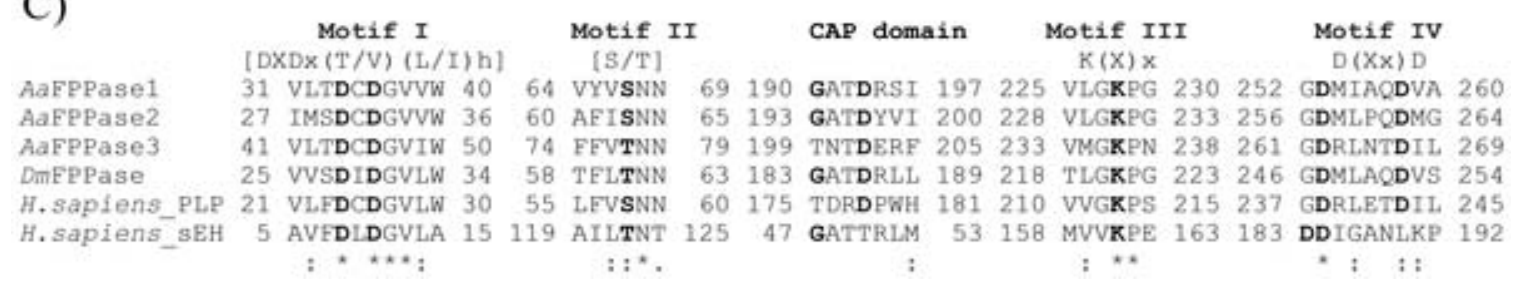

Figure 8: Homology model of the overall fold of AaFPPase-1 and amino acid sequence alignment of HAD motifs and cap domains from mosquito, fruit fly and human. (A) Homology model of the overall fold of AaFPPase-1. Core region is colored in green and cap region in light blue. Motifs are indicated by colors: motif I (red), motif II (orange), motif III (pink), motif IV (yellow), cap domain (dark blue) and squiggle (chocolate). B) Molecular surface diagram illustrating the active site pocket and the cap 2 region of AaFPPase-1. Core region is colored green and cap region in light blue. Motifs are indicated by colors: Motif I (red), motif II (orange), motif III (pink), motif IV (yellow), cap domain (dark blue) and squiggle (chocolate). The two structures were constructed by PyMOL using the Human pyridoxal phosphate phosphatase (2oycA) as template. C) Amino acid sequence alignment of HAD motifs and cap domains from mosquito (AaFPPase-1, -2 and -3), fruit fly (DmFPPase), human pyridoxal phosphatase $(H$. sapiens_PLP) and human epoxy hydrolase (H. sapiens_sEH). The suggested functions for the motifs are: motif I is required for nucleophilic attack, motif II is responsible for substrate binding, the motif III Lys is required for stabilizing the negative charge of the reaction intermediate together with the Ser/Thr of motif II, motif IV is needed for $\mathrm{Mg}^{2+}$ ion binding and the cap domain is involved in substrate recognition. Bold letters indicate the conserved residues in each motif. The numbers represent the amino acid positions in the sequences. " $h$ ' denotes a hydrophobic residue and " $x$ " any residue. Accession numbers: DmFPPase (CG15739), H. sapiens_PLP (NP_064711.1) and H. sapiens_sEH (NP_001243411.1). 


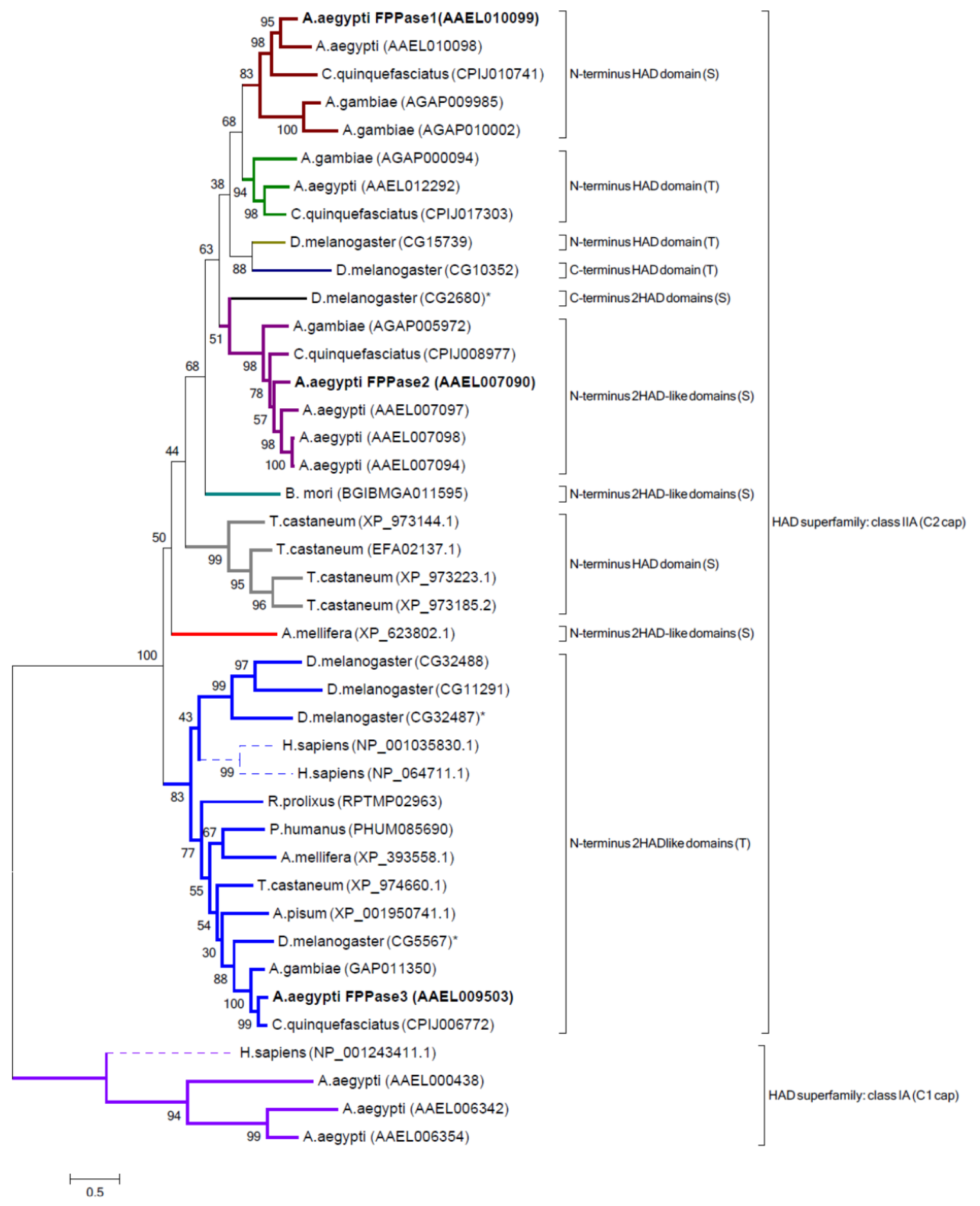

Figure 9: Phylogenetic analysis of HAD superfamily sequences from insects and human. Sequences are labeled with species names and accession numbers in between brackets. The bifunctional human epoxy hydrolase (NP_001243411.1) was used as outgroup. Sequences grouped in two clades. All sequences in Clade 1 are members of the NagD family included in the class IIA of HAD proteins. Sub-clades are separated by the 
localization of the HAD domain and the presence of a Ser (S) or Thr (T) in motif II. The position of the functional domain is referred as $\mathrm{N}$-terminus or C-terminus. Insects with two potential HAD functional domains are shown with an asterisk. Bold labels represent the AaFPPase- 1, AaFPPase-2 and AaFPPase-3. Human sequences are represented by dotted lines in the tree. All sequences in Clade 2 are epoxy hydrolases, which are members of the class IA of HAD proteins. 

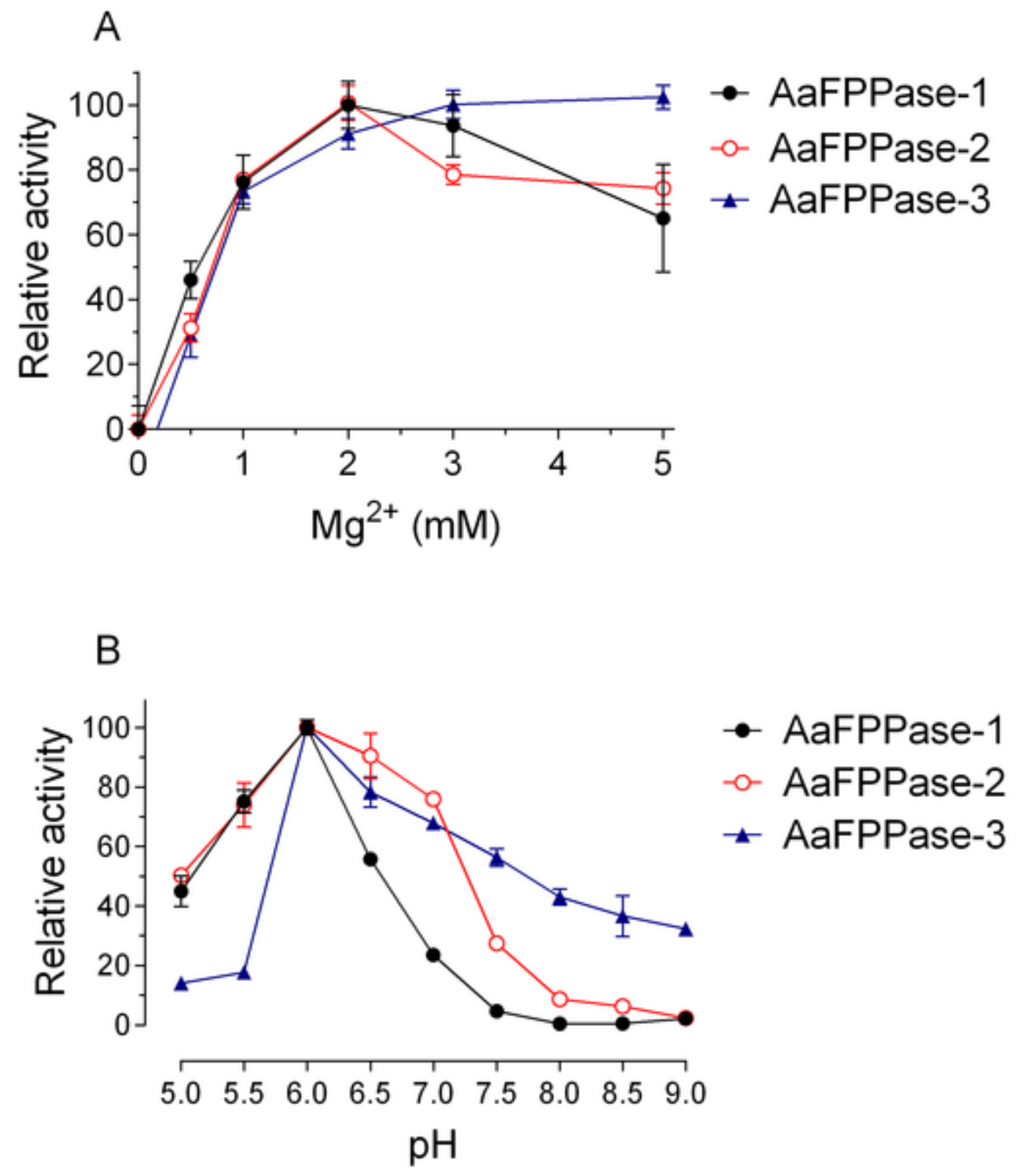

Figure 10: Metal dependence and optimum $\mathrm{pH}$ of AaFPPase. Phosphatase activity was measured using $p$-NPP. A) Magnesium dose-dependent increases of activities. B) Optimum $\mathrm{pH}$ determinations. Three different buffers were used: Sodium acetate at $\mathrm{pH} 4.5$ to 5.5, MES at $\mathrm{pH} 5.5$ to 7 and Tris at $\mathrm{pH} 7$ to 9 . Each value represents the means \pm S.E.M. of three replicate assays. Relative activity is defined as a percentage of the highest value recorded. 


$\begin{array}{llllll}\text { Substrate } & \begin{array}{l}K_{m} \\ (\mu \mathrm{M} \pm \mathrm{SE})\end{array} & \begin{array}{l}V_{\max } \\ \left(\mathrm{min}^{-1} \mathrm{mg}^{-1} \pm \mathrm{SE}\right)\end{array} & \begin{array}{l}K_{c a t} \\ \left(\mathrm{~s}^{-1}\right)\end{array} & \begin{array}{l}K_{c a t} / K_{m} \\ \left(\mathrm{M}^{-1} \mathrm{~s}^{-1}\right)\end{array} & \begin{array}{l}\text { Recombinant } \\ \text { enzymes }\end{array} \\ \text { FPP } & 222.36 \pm 11.0 & 6.45 \pm 0.76 & 3.33 & 1.5 \times 10^{4} & \text { AaFPPase-1 } \\ \text { GPP } & 184.45 \pm 14.16 & 12.71 \pm 0.37 & 7.92 & 4.3 \times 10^{4} & \text { AaFPPase-1 } \\ \text { IPP } & >900 & \mathrm{ND} & \mathrm{ND} & \mathrm{ND} & \text { AaFPPase-1 } \\ \text { FPP } & 221.02 \pm 15.62 & 5.77 \pm 0.15 & 2.98 & 1.32 \times 10^{4} & \text { AaFPPase-2 } \\ \text { GPP } & 273.98 \pm 2.52 & 28.3 \pm 0.95 & 17.49 & 6.3 \times 10^{4} & \text { AaFPPase-2 } \\ \text { IPP } & >900 & \mathrm{ND} & \mathrm{ND} & \mathrm{ND} & \text { AaFPPase-2 }\end{array}$

Table 2: Substrate specificity of AaFPPase-1 and AaFPPase-2 

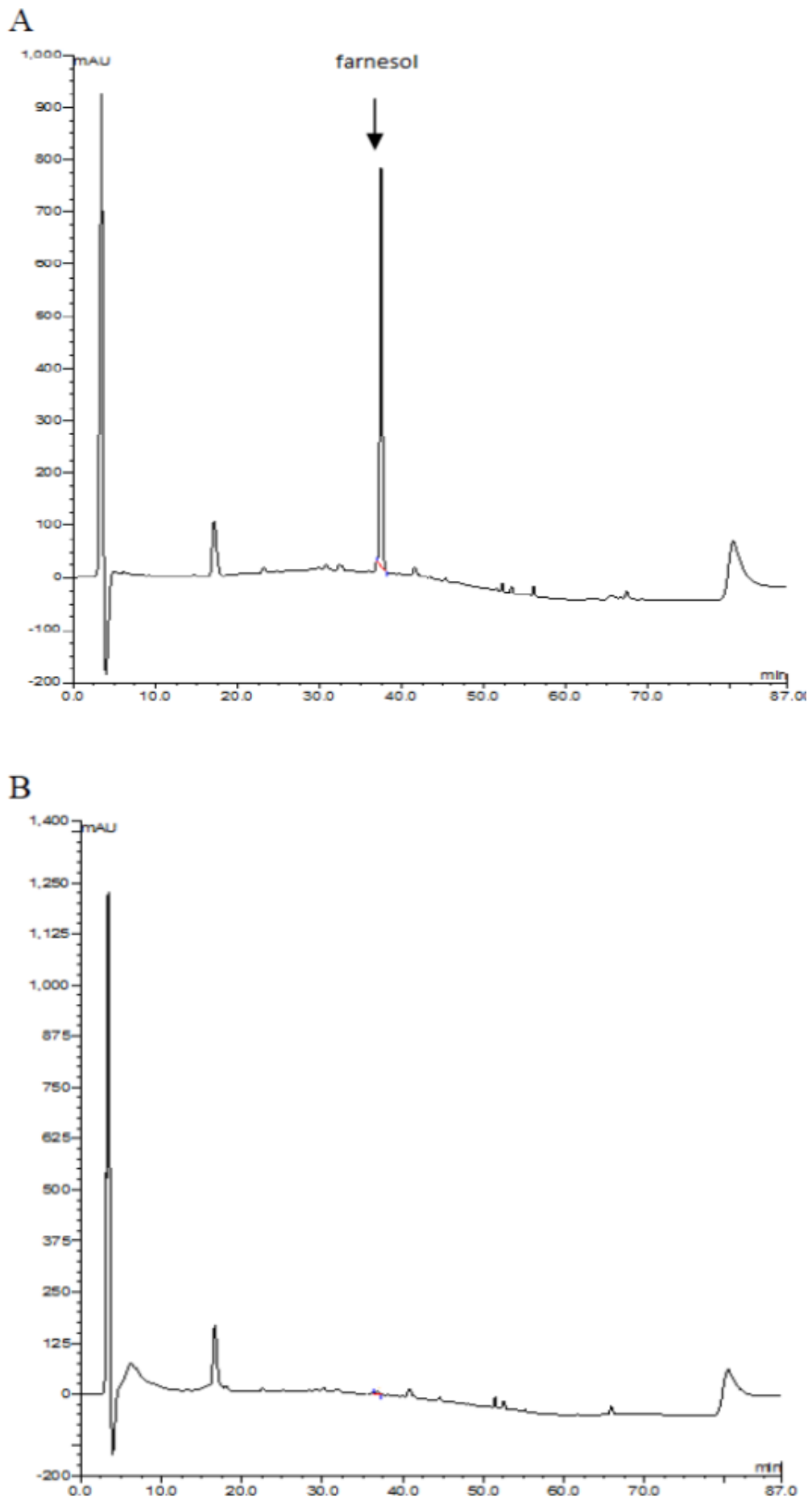

Figure 11: Chromatogram of a reverse-phase high performance liquid (HPLC) analysis showing the production of farnesol from FPP by AaFPPase-1. A) 300 $\mu \mathrm{M}$ FPP was incubated with AaFPPase- 1 in reaction buffer for $1 \mathrm{hr}$ at RT. Arrow indicates farnesol (retention time $37.5 \mathrm{~min}$ ). B) Negative control in which $1 \mathrm{mM}$ FPP was incubated in reaction buffer without adding enzyme for $1 \mathrm{hr}$ at RT. 


\section{AGGC}

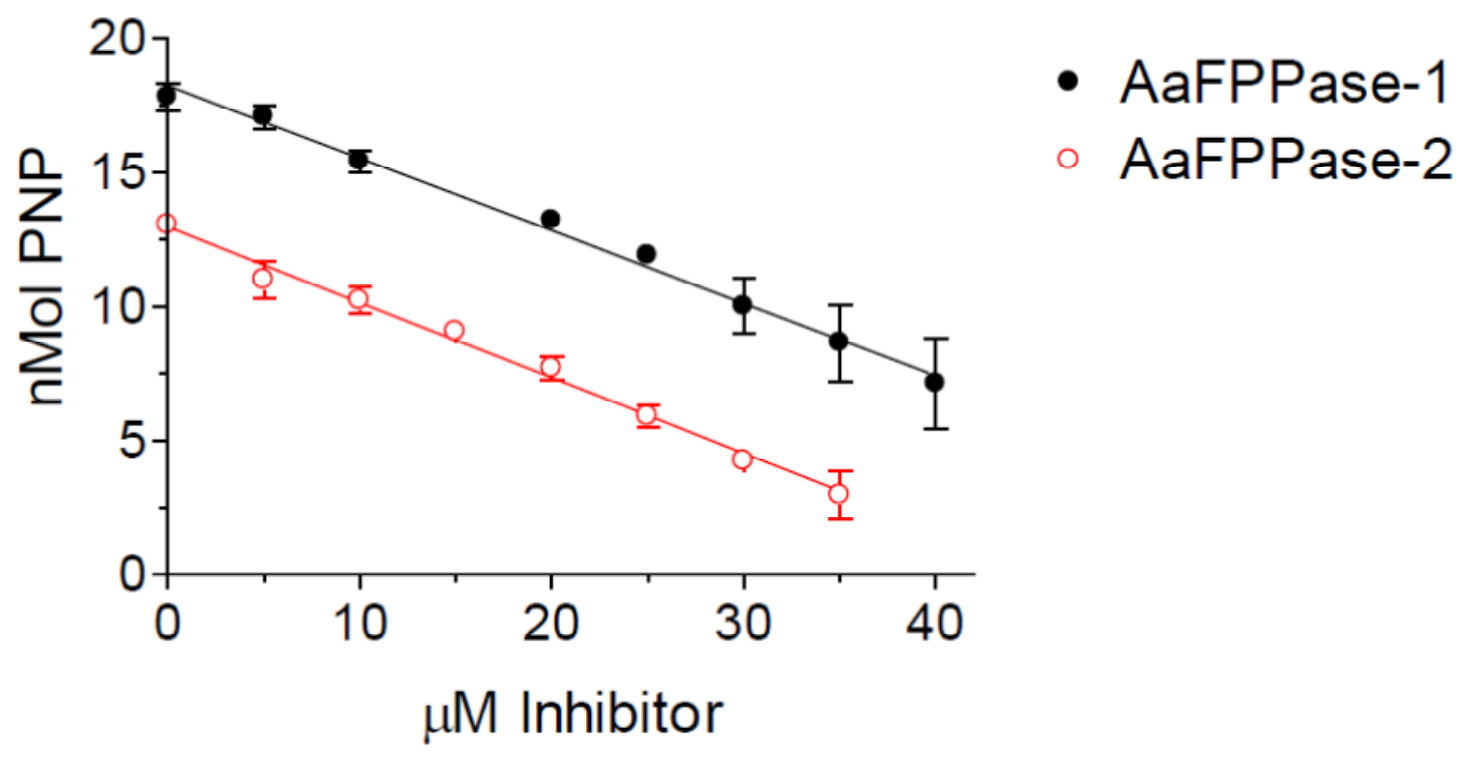

Figure 12: Effect of the inhibitor AGGC on AaFPPase activity. Recombinant AaFPPase-1 and -2 were pre incubated with different concentrations $(0$ to $40 \mu \mathrm{M})$ of N-acetyl-Sgeranylgeranyl-L-cysteine (AGGC) for $10 \mathrm{~min}$ and their activities were measured using the $p$-NPP assay. 


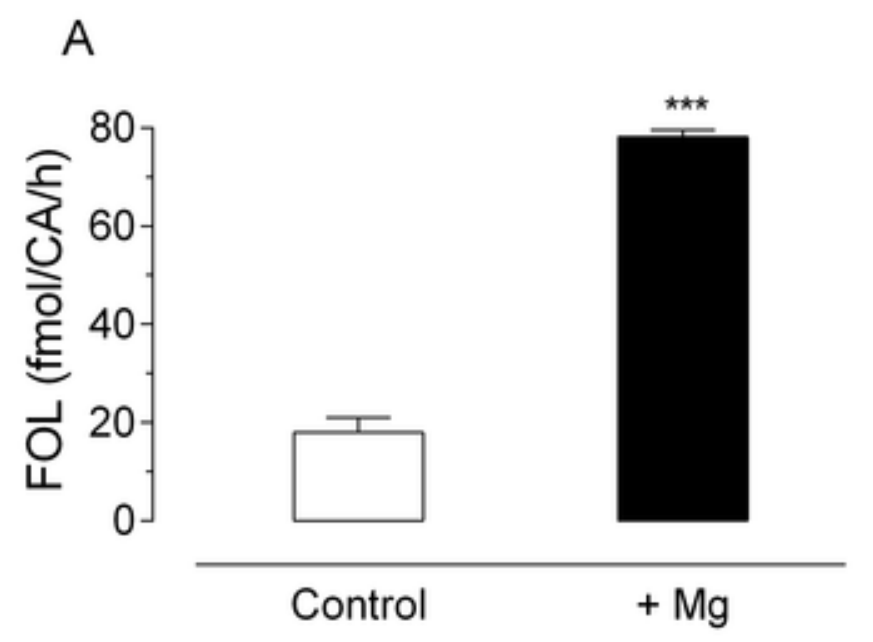

B

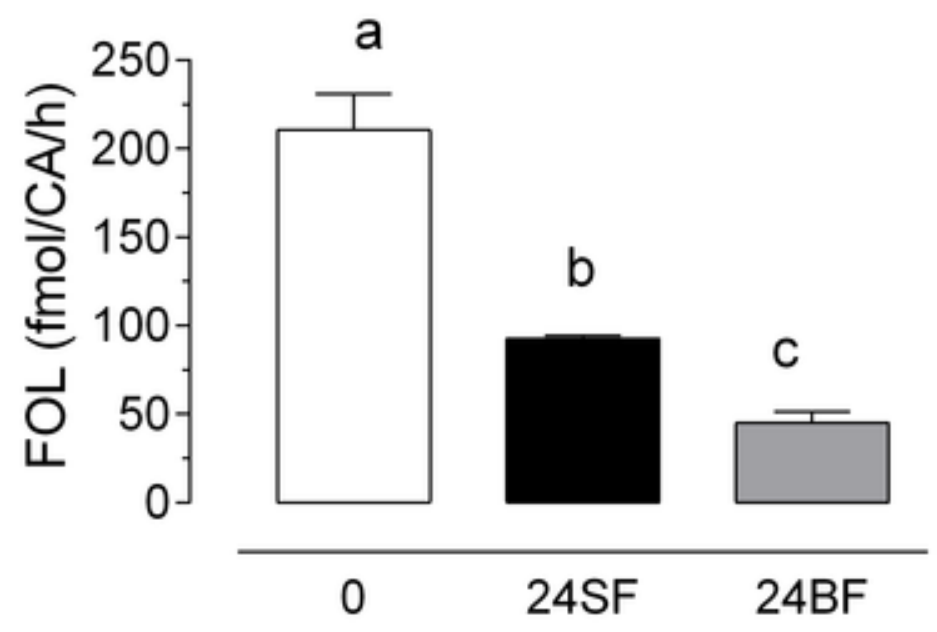

Figure 13: FPPase activity in CA extracts. A) Effect of $\mathrm{Mg}^{2+}$ on FPPase activity: Extracts of CA dissected from sugar-fed females $24 \mathrm{~h}$ after emergence were incubated with or without $2 \mathrm{mM} \mathrm{MgCl} 2$. Bars represent the means \pm S.E.M. of three replicates of extracts from groups of $5 \mathrm{CA}$. Asterisks denote significant difference (unpaired t-test, $* * * \mathrm{P}<0.001)$. B) The CA exhibited variable FPPase activity: Extracts of CA dissected from newly emerged females (0), $24 \mathrm{~h}$ after emergence (24SF) and $24 \mathrm{~h}$ after blood feeding (24BF) were incubated for $1 \mathrm{~h}$ in the presence of an excess of FPP. Bars represent the means \pm S.E.M. of three replicates of extracts from groups of $10 \mathrm{CA}$. Different letters above the columns indicate significant differences among treatments (one way ANOVA $p<0.05$, with Tukey's test of multiple comparisons). 


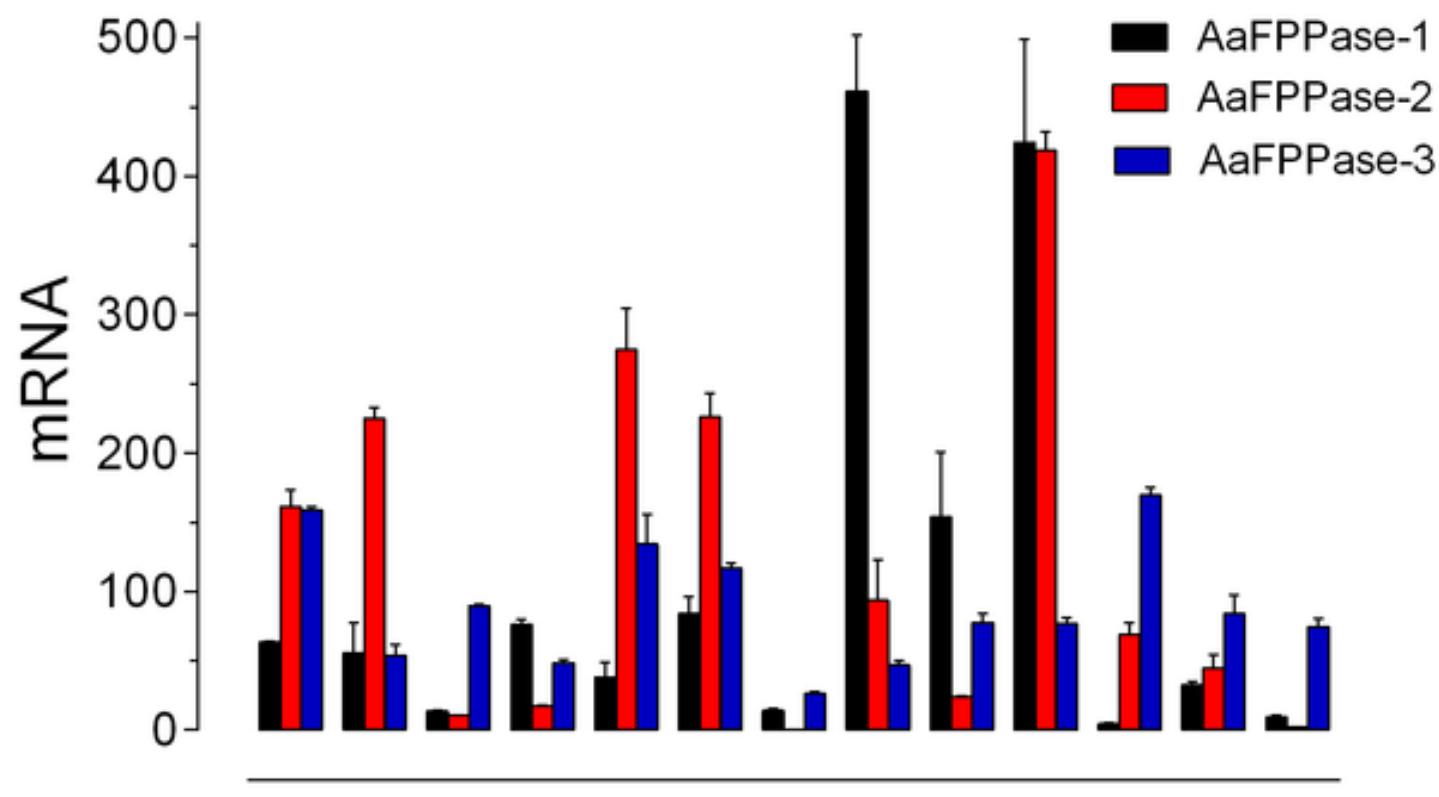

BR CA SG HT TG VG FB MG HG MT OV TS AG

\section{Figure 14: Tissue specific expression of AaFPPases}

All tissues were dissected from 3-day-old sugar-fed females, except for testis and accessory glands dissected from 3-day-old sugar-fed males. BR: brain; CA: corpora allata; SG: salivary gland; HT: heart; TG: thoracic ganglia; VG: ventral ganglia; FB: fat body; MG: midgut; HG: hindgut; MT: Malpighian tubules; OV: ovaries; TS: testis and AG: accessory gland. Each value represents the means \pm S.E.M of two independent biological replicates of 10-20 tissue samples evaluated in triplicate. AaFPPase mRNAs are expressed as copy number of mRNA/10,000 copies of rpL32 mRNA. 

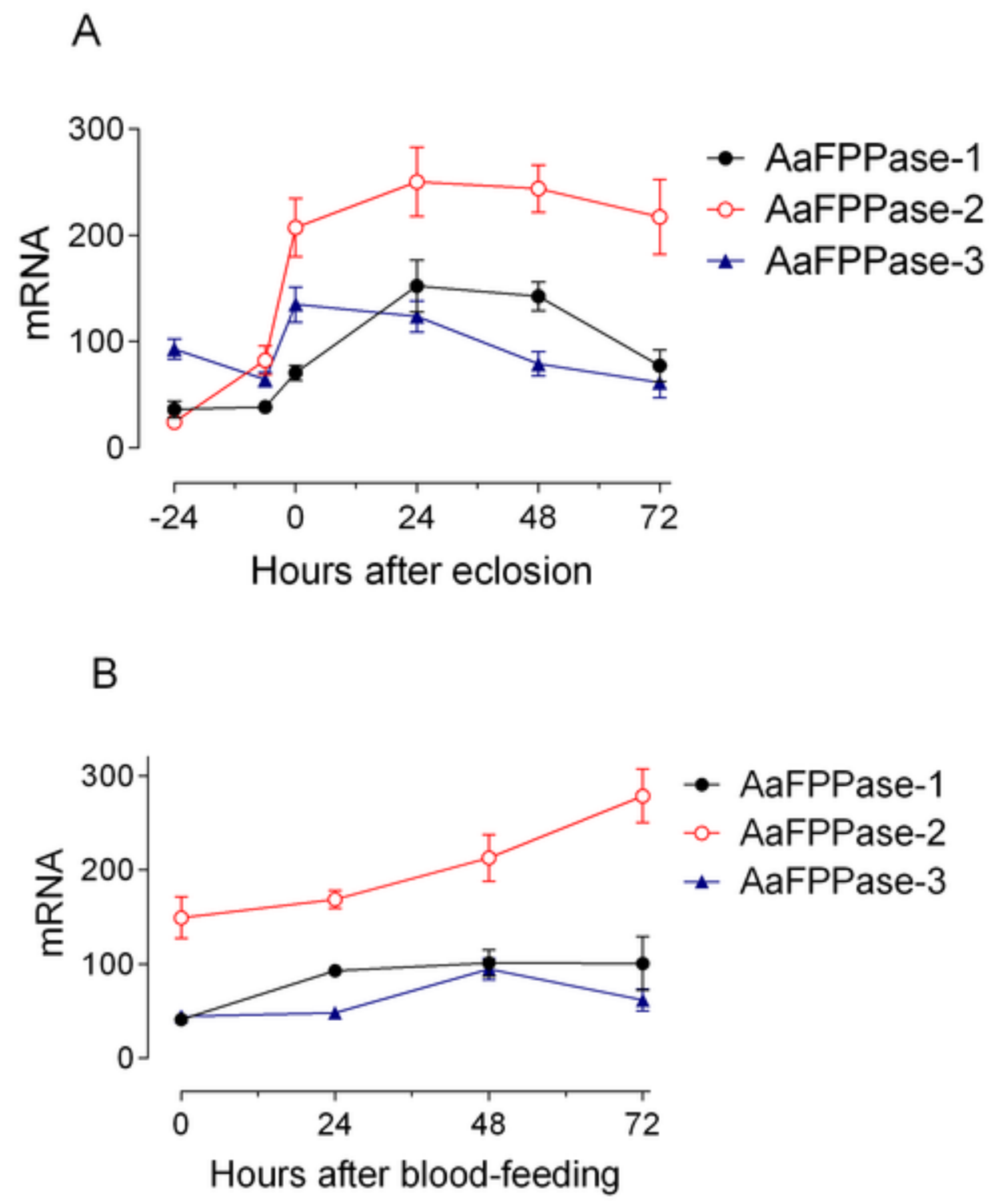

Figure 15: Developmental expression of AaFPPases. A) Expression on pupae and sugarfed females: mRNA was isolated from CA of pupae $24 \mathrm{~h}(-24)$ and $6 \mathrm{~h}$ before adult eclosion, newly emerged adult female $(0 \mathrm{~h})$, sugar-fed females 24,48 and $72 \mathrm{~h}$ after eclosion. B) Expression after blood feeding. Each data point is the means \pm S.E.M. of three independent biological replicates of $20 \mathrm{CA}$ evaluated in triplicate. AaFPPase mRNAs are expressed as copy number of mRNA/10,000 copies of rpL32 mRNA. 

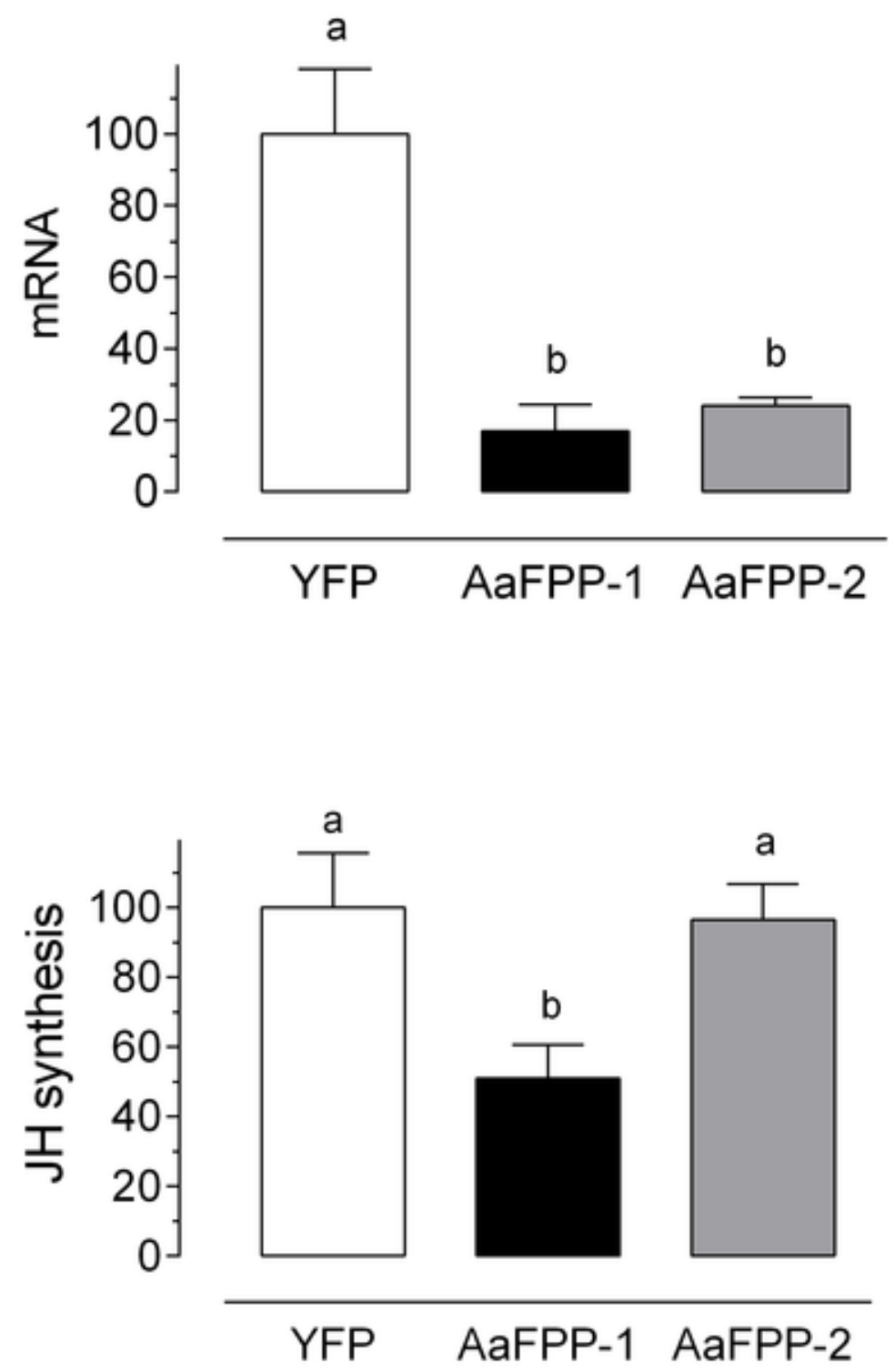

Figure 16: dsRNA mediated knockdown of AaFPPase-1 and -2. Newly emerged female mosquitoes were injected with dsAaFPPase-1, dsAaFPPase-2 or dsYFP; 4 days later transcript and $\mathrm{JH}$ levels were evaluated. A) Transcript levels are expressed as $\%$ of the YFP controls. Bars represent the means \pm S.E.M. of two replicates of RNA extracted from thoraxes. B) JH synthesized in vitro: CA were dissected from females injected with dsAaFPPase-1, dsAaFPPase-2 or YFP dsRNA and incubated in vitro for $4 \mathrm{~h}$. JH was evaluated by HLPC-FD. Bars represent the means \pm S.E.M. of four replicates of 4 CA. Different letters above the columns indicate significant differences among treatments (one way ANOVA $\mathrm{p}<0.05$, with Tukey's test of multiple comparisons). 


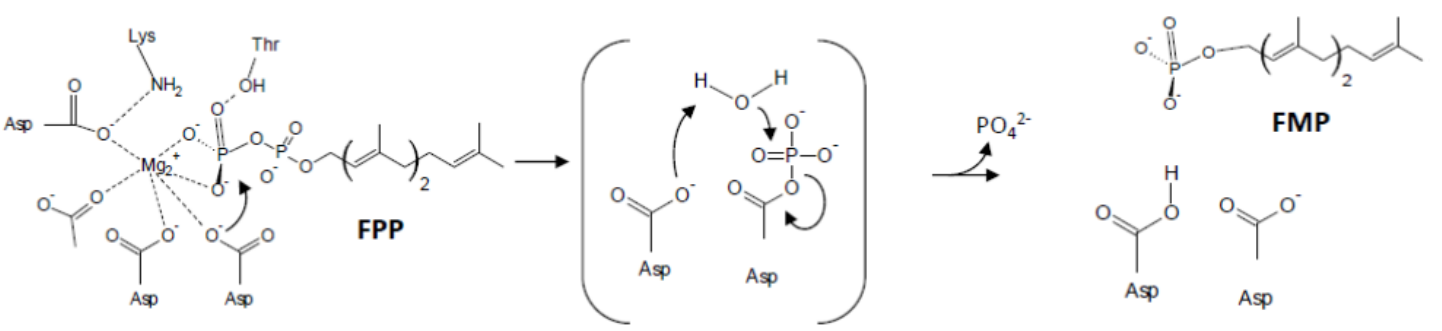

Enzyme -substrate complex. Nucleophilic attack by Asp on the phosphoryl group.

(2) Phosphoaspartyl enzyme-intermediate. Nucloephilic attack by water and release of the free phosphate.

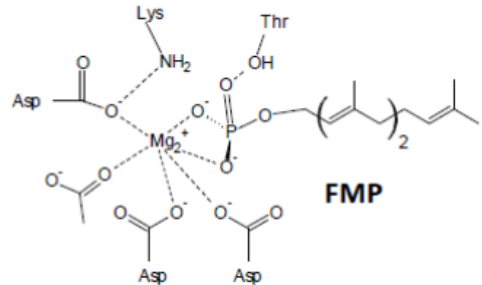

(4) Enzyme-substrate complex.

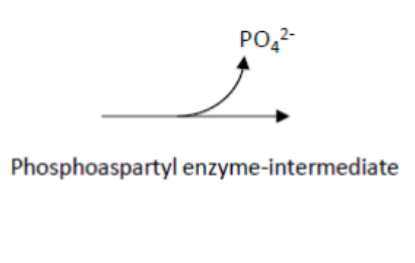

(5) Free enzyme. Enzyme returns to initial state.

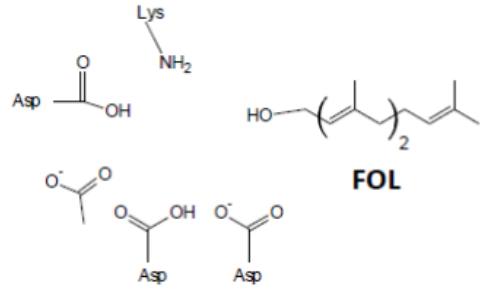

Figure 17: Schematic representation of the catalytic mechanism for AaFPPases. Catalysis proceeds through an aspartylphosphate intermediate. 1) Once the FPP is bound, the $\mathrm{Mg}^{2+}$ ion in the active site interacts with the negatively charged phosphate, preparing it for nucleophilic attack by the first conserved aspartate on motif I. 2) As a result, an acyl phosphate intermediate is formed with the carboxyl group of this aspartate and a water molecule is deprotonated by the second aspartate of motif I; hydrolyzing the acyl phosphate intermediate and returning the enzyme to the native state 3) The enzyme forms a new complex with FMP. 4) Catalysis of FMP occurs again through an aspartylphosphate intermediate. 5) Farnesol is released and the enzyme returns to the initial state. 


\subsection{References}

1. Allen KN, Dunaway-Mariano D (2004) Phosphoryl group transfer: evolution of a catalytic scaffold. TRENDS Biochem Sci 29:495-503.

2. Allen KN, Dunaway-Mariano D (2009) Markers of fitness in a successful enzyme superfamily Curr Opin Struct Biol 19:658-665.

3. Aravind L, Galperin MY, Koonin EV (1998) The catalytic domain of the P-type ATPase has the haloacid dehalogenase fold. TIBS 23:127-129.

4. Arnold K, Bordoli L, Kopp J, Schwede T (2006) The SWISS-MODEL Workspace: A web-based environment for protein structure homology modeling. Bioinformatics 22:195-201.

5. Burroughs AM, Allen KN, Dunaway-Mariano D, Aravind L (2006) Evolutionary genomics of the HAD superfamily: understanding the structural adaptations and catalytic diversity in a superfamily of phosphoesterases and allied enzymes. J Mol Biol 361:1003-1034.

6. Cao L, Zhang P, Grant DF (2009) An insect farnesyl phosphatase homologous to the $\mathrm{N}$-terminal domain of soluble epoxide hydrolase. Biochem Biophys Res Comm 380:188-192.

7. Cronin A, Mowbray S, Durk H, Homburg S, Fleming I, et al. (2003) The N-terminal domain of mammalian soluble epoxide hydrolase is a phosphatase. Proc Natl Acad Sci U S A 100:1552-1557.

8. Jakoby WB, Ziegler DM (1990) The enzymes of detoxication. J Biol Chem 265:20715-20718.

9. Joo JH, Jetten AM (2010) Molecular mechanisms involved in farnesol-induced apoptosis. Cancer Lett 287:123-135.

10. Joune F, Laurent G, Chaboteaux C, Nonclercq D, Durbecq V, et al. (2008) Farnesol, a mevalonate pathway intermediate, stimulates MCF-7 breast cancer cell growth through farnesoid-X-receptor-mediated estrogen receptor activation. Breast Cancer Res Treat 107:49-61. 
11. Kinjoh T, Kaneko Y, Itoyama K, Mita K, Hiruma K, et al. (2007) Control of juvenile hormone biosynthesis in Bombyx mori: cloning of the enzymes in the mevalonate pathway and assessment of their developmental expression in the corpora allata. Insect Biochem Molec Biol 37:808-818.

12. Klowden MJ (1997) Endocrine aspects of mosquito reproduction. Arch Insect Biochem Physiol 35:491-512.

13. Koonin EV, Tatusov RL (1994) Computer analysis of bacterial haloacid dehalogenases defines a large superfamily of hydrolases with diverse specificity. Application of an iterative approach to database search. J Mol Biol 244:125-132.

14. Lahiri SD, Zhang G, Dai J, Dunaway-Mariano D, Allen KN (2004) Analysis of the substrate specificity loop of the HAD superfamily cap domain. Biochem 43:28122820.

15. Lawson D, Arensburger P, Atkinson P, Besansky NJ, Bruggner RV, et al. (2009) VectorBase: a data resource for invertebrate genomics. Nucleic Acids Res 37:D583D587.

16. Li YP, Hernandez-Martinez S, Unnithan GC, Feyereisen R, Noriega FG (2003) Activity of the corpora allata of adult female Aedes aegypti: Effects on mating and feeding. Insect Biochem Mol Biol 33:1307-1315.

17. Mayoral JG, Nouzova M, Navare A, Noriega FG (2009) NADP ${ }^{+}$-dependent farnesol dehydrogenase, a corpora allata enzyme involved in juvenile hormone synthesis. Proc Natl Acad Sci U S A 106:21091-21096.

18. Mayoral JG, Nouzova M, Yoshiyama M, Shinoda T, Hernandez-Martinez S, et al. (2009) Molecular and functional characterization of a juvenile hormone acid methyltransferase expressed in the corpora allata of mosquitoes. Insect Biochem Mol Biol 39:31-37.

19. Noriega FG (2004) Nutritional regulation of JH synthesis: a mechanism to control reproductive maturation in mosquitoes? Insect Biochem Mol Biol 34:687-693.

20. Nouzova M, Edwards MJ, Mayoral JG, Noriega FG (2011) A coordinated expression of biosynthetic enzyme controls the flux of juvenile hormone precursors in the corpora allata of mosquitoes. Insect Biochem Mol Biol 9:660-669. 
21. Pechlivanis M, Kuhlmann J (2006) Hydrophobic modifications of Ras proteins by isoprenoid groups and fatty acids-More than just membrane anchoring. Bioch Bioph Acta 1764:1914-1931.

22. Perez-Hedo M, Rivera-Perez C, Noriega FG (2013) The Insulin/TOR signal transduction pathway is involved in the nutritional regulation of juvenile hormone synthesis in Aedes aegypti. Insect Biochem Molec Biol 43:495-500.

23. Rivera-Perez C, Nouzova M, Clifton ME, Martin Garcia E, LeBlanc E, et al. (2013) Aldehyde dehydrogenase 3 converts farnesal into farnesoic acid in the corpora allata of mosquitoes. Insect Biochem Molec Biol 43:675-682.

24. Rivera-Perez C, Nouzova M, Noriega FG (2012) A quantitative assay for the juvenile hormone and their precursors using fluorescent tags. PLoS One 7(8):e43784. doi:10.1371/journal.pone.0043784.

25. Rizzo WB, Craft DA (1991) Sjogren-Larsson syndrome. Deficient activity of the fatty aldehyde dehydrogenase component of fatty alcohol:NAD ${ }^{+}$oxidoreductase in cultured fibroblast. J Clin Invest 88:1643-1648.

26. Roullet JB, Spaetgens RL, Burlingame T, Feng ZP, Zamponi GW (1999) Modulation of Neuronal Voltage-gated Calcium Channels by Farnesol. J Biol Chem 274:2543925446.

27. Schwede T, Kopp J, Guex N, Peitsch MC (2003) SWISS-MODEL: an automated protein homology-modeling server. Nucleic Acids Res 31:3381-3385.

28. Seifried A, Schultz J, Gohla A (2013) Human HAD phosphatases: structure, mechanism, and roles in health and disease. FEBS J 280:549-71.

29. Tamura K, Peterson D, Peterson N, Stecher G, Nei M, et al., (2011) MEGA5: Molecular evolutionary genetics analysis using maximum likelihood, evolutionary distance, and maximum parsimony methods. Mol Biol Evol 28:2731-2739.

30. Tschantz WR, Digits JA, Pyun HJ, Coates RM, Casey PJJ (2001) Lysosomal prenylcysteine lyase is a FAD-dependent thioether oxidase. J Biol Chem 276:23212324. 
31. Ueda H, Shinoda T, Hiruma K (2009) Spatial expression of the mevalonate enzymes involved in juvenile hormone biosynthesis in the corpora allata in Bombyx mori $\mathrm{J}$ Insect Physiol 55:798-804.

32. Van Veldhoven PP, Mannaerts GP (1987) Inorganic and organic phosphate measurements in the nanomolar range. Anal Biochem 161:45-48. 


\section{Chapter 3: Structural and biochemical characterization of a mevalonate kinase involved in juvenile hormone pathway in Aedes aegypti}

\subsection{Abstract}

Mevalonate kinase catalyzes the ATP-dependent phosphorylation of mevalonic acid to form mevalonate 5-phosphate, a key intermediate in the juvenile hormone (JH) pathway. Here we report the expression, biochemical and structural characterization of an Aedes aegypti mevalonate kinase (AaMK) enzyme expressed in the corpora allata. Different isoprenoids were analyzed as inhibitors of the recombinant enzyme using a traditional spectrophotometric assay and a HPLC assay. We found that AaMK was strongly inhibited by long chain isoprenoids pyrophosphates including the 20-carbon geranyl-geranyl pyrophosphate (GGPP), the 15-carbon farnesyl pyrophosphate (FPP) and the 10-carbon geranyl pyrophosphate (GPP), all of them in the nanomolar range. Short chain isoprenoids

pyrophosphates such as the 5-carbon compounds isopentenyl pyrophosphate (IPP) and dimethylallyl pyrophosphate (DMAPP) also inhibited but only in the micro molar range. Other precursors of the $\mathrm{JH}$ biosynthesis pathway such as phospho mevalonate (PM), diphospho mevalonate (DPM) and farnesol (FOL) were not inhibitors of MK activity. In addition we also found a feedback inhibition of AaMK activity by FPP and GPP in mosquito crude extracts.

\subsection{Introduction}

Mevalonate kinases (MK) (EC 2.7.1.36) catalyze the synthesis of phosphomevalonate (PM) by transferring the $\gamma$-phosphoryl group from ATP to the C5 hydroxyl oxygen of mevalonic acid (MA) (PM) (Fu et al., 2002). The irreversible reaction requires a divalent cation and represents a key step in the production of juvenile hormone 
(JH) in insects (Noriega, 2014). Originally described in the late 1950's for its role on cholesterol synthesis (Amdur et al., 1957), MK is also involved in the synthesis of a diverse group of non-sterol isoprenoid metabolites important in various cellular functions; such as protein prenylation, protein glycosylation and cell cycle regulation (Belles et al., 2005). In addition MK dysfunctions are responsible for human inherited diseases, such as mevalonic aciduria and hyperimmunoglobulinemia $\mathrm{D}$ /periodic fever syndrome (Hoffman et al., 1986).

Mevalonate kinases are found in the three domains of life (Lombard and Moreira, 2010). They are members of the "GHMP kinase family", a group of sugar kinase that originally included galactokinases, homoserine kinases, mevalonate kinases, and phosphomevalonate kinases (Bork et al., 1993; Cheek et al., 2002). The GHMP kinase family has been now extended to include other kinases such as archaea shikimate kinases, L-threonine kinases, $\mathrm{N}$-acetylgalactosamine kinases, glucuronokinases, arabinose kinases, galacturonic acid kinases, mevalonate diphosphate decarboxylases (MDD), and 4-(cytidine 5-diphospho)-2-Cmethyl- D-erythritol kinases (Cheek et al., 2002). Members of the GHMP kinase family have a number of conserved amino acid signature motifs, which are involved in the binding of ATP-Mg, as well as the substrates to be phosphorylated (Houten et al., 2000; Fu et al., 2002).

Mevalonate kinases from different organisms have a homodimeric structure in solution, which is composed of identical subunits with a molecular weight ranging from 70 to $105 \mathrm{KDa}$ (Yang et al., 2002; Fu et al., 2002; Andreassi et al., 2007). Kinetic studies suggest that the enzyme catalyzes an ordered sequential reaction, with mevalonate binding first to the enzyme, and with PM as the first product released after catalysis (Beytia et al., 
1970; Fu et al., 2002). Above mechanism of reaction has been postulated for the rat MK (Rattus norvegicus MK or RnMK) (Fu et al., 2002). The structure of the RnMK shows that the C5-hydroxyl group of the MA and the $\gamma$-phosphate group of ATP are located close to Aspartic acid ${ }_{204}$ that makes a salt bridge with Lysine ${ }_{13}$. In the complex, penta-coordinated $\gamma$-phosphate transition state is stabilized by the magnesium ion, the side chains of Glutamic $\operatorname{acid}_{193}$ and Lysine ${ }_{13}$, as well as the main-chain carbonyl group of Serine ${ }_{146}$. Aspartic acid 204 act as a base, abstracting a proton from a hydroxyl group in the MA. This converts MA into an excellent nucleophile, which then attacks the $\gamma$-phosphorus of ATP. Lysine ${ }_{13}$ is believed to maintain the aspartate residue in the deprotonated state and decrease its $\mathrm{p} K_{a}$ to facilitate the proton transfer (Fu et al., 2002). The "catalytic base mechanism" is supported by site-directed mutagenesis studies. In the Homo sapiens MK (HsMK), a mutation of the critical aspartate residue (catalytic base) decreases the activity by ten thousand fold compared with the wild type (Potter and Miziorko, 1997). Similarly, the catalytic rate is reduced by a modification of the lysine residue (Potter and Miziorko, 1997; Potter et al., 1997).

Another important feature of this enzyme is that its activity is regulated by feedback inhibition by isoprenoids, such as farnesyl pyrophosphate (FPP) and geranyl pyrophosphate (GPP). The inhibition has been described for several MKs, including Homo sapiens MK (Hinson et al., 1999), Rattus norvegicus MK, (Tanaka et al., 1990) and Sus domesticus MK (Beytia et al., 1970). A similar type of inhibition is also observed for MK of plants, including Phaseolus vulgaris, Cucumis melo and Hevea latex (Gray and Kekwick, 1972), yeast such as Saccharomyces cerevisia (Oulmouden and Karst, 1991), bacteria such as Staphylococcus aureus (Voynova et al., 2003) and archaea such as 
Methanococcus jannaschii (Huang et al., 1999). Isoprenoids are potent competitive inhibitors for the binding of ATP to MKs (Hinson et al., 1997; Potter and Miziorko, 1997). Sensitivity to feedback inhibition is much greater in eukaryotic enzymes than bacterial or archaeal enzymes (Fu et al., 2008; Primak et al., 2011). On the basis of their feedback inhibition, MKs can be classified into at least three different classes: 1) class I MKs: showing feedback inhibition by isoprenoids, but not by diphosphomevalonate (DPM), 2) class II MKs: which do not show feedback inhibition by isoprenoids, but are strongly inhibited by DPM, and 3) class III MKs: that do not show feedback inhibition by either isoprenoids or DPM (Primak et al., 2011).

We have characterized a mevalonate kinase activity from the CA of Aedes aegypti (AaMK). Similarly to other animal MKs, recombinant $A a \mathrm{MK}$ displays a strong feedback inhibition by long chain isoprenoids, such as geranyl-geranyl pyrophosphate (GGPP), FPP and GPP; with Ki values of less than $1 \mu \mathrm{M}$. The endogenous activity of AaMK was also strongly inhibited by adding long chain isoprenoids to the crude extract of mosquito thoraces (containing the CA). Homology modeling was used to build the structure of AaMK in order to elucidate the mechanism of reaction and feedback inhibition by isoprenoids.

\subsection{Materials and methods}

\subsubsection{Chemicals}

Geranyl-geranyl pyrophosphate (GGPP), farnesyl pyrophosphate (FPP), geranyl pyrophosphate (GPP), isopentenyl pyrophosphate (IPP), dimethyl allyl pyrophosphate (DMAPP) and farnesol (FOL) were purchased from Echelon Biosciences (Salt Lake City, UT). Mevalonic acid (MA), phosphomevalonate (PM), diphosphomevalonate (DPM), 
phosphoenolpyruvate (PEP) and nicotinamide adenine dinucleotide reduced (NADH) were purchased from Sigma-Aldrich (St. Louis, MO). Pyruvate kinase (PK) and lactate dehydrogenase (LDH) were purchased from LEE bioscience.

\subsubsection{Insects}

Aedes aegypti of the Rockefeller strain were reared at $28{ }^{\circ} \mathrm{C}$ and $80 \%$ relative humidity under a photoperiod of $16 \mathrm{~h}$ light: $8 \mathrm{~h}$ dark. A cotton pad soaked in 3\% sucrose solution was provided to adults.

\subsubsection{Secondary and tertiary structure of AaMK}

The secondary structure was predicted online using the ExPASy web tools (Peter $e t$ al., 1974). The mosquito deduced amino acid sequence and those selected from other insect species were aligned using ClustalW (Thompson et al., 1997); the MK from Rattus norvegicus was included in the alignment to identify some of the conserved motifs that characterize this group of enzymes.

The three dimensional model structure for AaMK was predicted using the crystal structure of Human MK (Protein Data Bank ID code 2r3v.3) as a template. The identity of the model and $A a \mathrm{MK}$ was $37.75 \%$. The model was performed using the protein structure homology modeling server Swiss v.8.05 (Schwede et al., 2003; Arnold et al., 2006)

\subsubsection{Expression of recombinant AaMK}

The AaMK cDNA was expressed in E. coli cells as described by (Nyati et al., 2013). Recombinant His-tagged proteins were purified using HiTrap chelating columns and PD-10 desalting columns (Amersham Pharmacia, Piscataway, NJ). Glycerol was added to the enzyme solution (final concentration $50 \%$ ), and samples were stored at $-20{ }^{\circ} \mathrm{C}$ until 
used. Protein concentrations were determined using the bicinchoninic acid protein assay reagent

(BCA) (Pierce, Rockford, IL). Bovine serum albumin was used as a standard.

\subsubsection{Enzyme assays}

\subsubsection{Enzyme coupled spectrophotometric assay}

The catalytic activity of the $A a \mathrm{MK}$ was measured using a modified spectrophotometric assay that couples ADP formation to pyruvate synthesis and reduction to lactate (Primak et al., 2011). The initial rate of disappearance of NADH serves as a measurement of the phosphorylation of MA by MK. The assays were performed in triplicate in 96-well plates (BioTek, Winooski, VT) for $10 \mathrm{~min}$ at $30{ }^{\circ} \mathrm{C}$. Each $100 \mu \mathrm{l}$ of reaction mixture contained $0.5 \mathrm{mM}$ phosphoenolpyruvate, $0.01 \mathrm{mM}$ DTT, $0.35 \mathrm{mM}$ NADH, $10 \mathrm{mM} \mathrm{MgCl} 2,2 \mathrm{U}$ of LDH, and $2 \mathrm{U}$ of PK in $100 \mathrm{mM}$ Tris-HCl pH 7.6.

The Michaelis-Menten constant, $K_{m-M A}$ was determined at a saturating concentration of ATP (5 mM); with MA concentrations ranging from 0.005 to $2.5 \mathrm{mM}$. The reactions were initiated with the addition of $150 \mathrm{ng}$ of recombinant $A a \mathrm{MK}$. The $K_{m-A T P}$ was determined using saturating concentrations of MA $(1.25 \mathrm{mM})$ and ATP concentrations ranging from 0.005 to $5 \mathrm{mM}$. The amount of NADH oxidized to $\mathrm{NAD}^{+}$was monitored continuously at $340 \mathrm{~nm}$. Absorbance changes were plotted against time to determine the rates of the MK-coupled reactions. To determine steady-state kinetic parameters, data were subjected to nonlinear regression fits to the Michaelis-Menten equation using the GraphPad Prism software (San, Diego, CA).

The AaMK inhibition studies were performed in triplicate by adding to the reaction mix precursors of JH synthesis (DPM, DMAPP, IPP, GPP and FPP), as well as GGPP at 
various concentrations. To determine the $K_{i}$ value for GPP, FPP and GGPP, we used the multicurve fits of the GraphPad Prism software.

\subsubsection{RP-HPLC analysis of the products of MK catalysis.}

The phosphorylation of MA into PM was analyzed by reverse-phase HPLC. MA $(200 \mu \mathrm{M})$ and ATP $(250 \mu \mathrm{M})$ were incubated with recombinant AaMK for $60 \mathrm{~min}$ in the reaction buffer (100 mM Tris- $\mathrm{HCl} \mathrm{pH} 7.5,10 \mathrm{mM} \mathrm{MgCl} 2,0.5 \mathrm{mM}$ DTT). Reactions were

terminated by adding $500 \mu \mathrm{l}$ of acetonitrile, and then vortex $1 \mathrm{~min}$. Samples were centrifuged at 14,000 rpm for $5 \mathrm{~min}$ and the organic phase was recovered, filtered and analyzed by reverse-phase HPLC on a Dionex Summit System (Dionex, Sunnyvale, CA) as previously described (Nyati et al., 2013). Water or/and glycerol were used in place of recombinant enzyme in negative controls.

\subsubsection{MK activity in the crude extract (CE) of mosquitoes}

Mevalonate kinase activities from mosquito female thoraxes were measured by monitoring the production of PM using RP-HPLC. Thoraxes from $24 \mathrm{~h}$ old $3 \%$ sugar-fed females were dissected in Aedes saline solution and transferred to a buffer solution (100 $\mathrm{mM}$ Tris- $\mathrm{HCl} \mathrm{pH} 7.5,10 \mathrm{mM} \mathrm{MgCl}, 0.01 \mathrm{mM}$ DTT). Thoraxes were homogenized for 1 min, sonicated $3 \mathrm{~min}$ and centrifuged at $10,000 \mathrm{~g}$ for $10 \mathrm{~min}$ at $4{ }^{\circ} \mathrm{C}$. Supernatants were recovered and used as crude extract (CE) for activity assays as previously described (Nyati et al., 2013). The protein contents of the CE were measured by BCA assay. Enzymatic assay was performed as previously described using $4 \mathrm{mg}$ of protein. Controls such as boiled crude extract and reactions without enzyme were included. A standard curve was constructed for the quantification of PM. 


\subsubsection{Statistical analysis}

Statistical analyses were performed using the GraphPad Prism Software (San Diego, CA, USA). The results are expressed as means \pm S.E.M. Significant differences (P $<0.05)$ were determined with one-way ANOVA followed by a pair-wise comparison of means (Tukey’s test).

\subsection{Results}

\subsubsection{Molecular and structural characterization of $A$. aegypti mevalonate kinase}

A single orthologue gene to the AaMK EST was found in the genome of A. aegypti (VectorBase) (Lawson et al., 2009). The AaMK gene (Vectorbase accession Num. AAEL006435) is located on supercontig 1.205, and it is composed of three exons interrupted by two introns with lengths of 68 and 57 bp. It encodes a 397 amino acid protein with an estimated molecular weight of $43.27 \mathrm{kDa}$.

Analysis of the AaMK structure revealed a fold consisting of a mixture of $\alpha$-helices and $\beta$-sheets, which are organized into $\mathrm{N}$ - terminal (include amino acids 1 to 246, 358 to 397) and C-terminal (include amino acids 247 to 357) domains arranged in a V-shape that creates a central cleft, with the active site located at the base of the cleft (Fig 18). The Nterminal domain (blue in Fig. 18) is composed of ten $\beta$ sheets ( $\beta 1-\beta 9$ and $\beta 12)$ and eight $\alpha$ helices ( $\alpha 1-\alpha 7$ and $\alpha 12$ ). The C-terminal domain (green in Fig. 18) is composed of four helices $(\alpha 8-\alpha 11)$ and two $\beta$ sheets $(\beta 10$ and $\beta 11)$ (Fig. 18). A similar structure was previously described for other MKs (Yang et al., 2002; Fu et al., 2002; Andreassi et al., 2007).

Amino acid sequence alignments of MKs from seven insect species including Aedes aegypti (AaMK), Culex quinquefasciatus (CqMK), Anopheles gambiae (AmMK), 
Drosophila melanogaster (DmMK), Bombyx mori (BmMK), Danaus plexippus (DpMK), Apis mellifera (AmMK) and Acyrthosiphon pisum (ApMK) (with similarities between 36\% and 44\%) revealed the four well conserved motifs typical of MKs (Fig. 19). The Rattus norvegicus MK, a protein with a solved crystal structure (Fu et al., 2002) and a close similarity with the sequence of $A a \mathrm{MK}(31 \%)$ was also included in the analysis.

\subsubsection{Expression and purification of recombinant A. aegypti mevalonate kinase}

Expression of the recombinant His-AaMK protein was induced in the E. coli Rosetta strain (DE3 cells) using the pET28 vector. AaMK was purified from the soluble fraction by immobilized metal affinity chromatography; yielding a protein with a molecular weight consistent with the estimated molecular weight of $43.27 \mathrm{kDa}$ (Fig. 21). It has been previously reported that inclusion of the His tag had no effect on the kinetics properties of Homo sapiens MK (HsMK) (Hinson et al., 1997) and RnMK (Chu et al., 2007); therefore the recombinant His-AaMK was used to study its enzymatic properties without cleavage of the His tag.

\subsubsection{Mevalonate kinase activity}

Mevalonate kinase activity was measured by using both the enzyme-coupled assay and the HPLC method (Fig. 22). The catalytic activity of AaMK increased in a dose response manner when $\mathrm{Mg}^{2+}$ was used as a cofactor. $\mathrm{Mn}^{2+}$ and $\mathrm{Co}^{2+}$ also enhanced $\mathrm{MK}$ activity to a lesser degree than $\mathrm{Mg}^{2+}$ (Fig. 23). The AaMK activity was investigated at different pHs. The optimum $\mathrm{pH}$ was found to be 7.5 to 8.0 ; with the enzyme exhibiting 60$70 \%$ of its optimum activity over a rather broad $\mathrm{pH}$ range (7 to 8.5 ) (Fig. 23). To determine the nucleotide specificity of MK, we first investigated the rates at which pyruvate kinase (PK) could use nucleoside diphosphates other than ADP. The activity of PK was measured 
by coupling the nucleotide diphosphate-dependent production of pyruvate and reduction to lactate catalyzed by lactate dehydrogenase (LDH). Relative rates measured for three replicate assays for ADP, GDP, UDP and CDP were respectively 100, 70, 40 and 20 percent. We utilized the enzyme coupled assay to determine catalytic rates in the presence of different triphosphates phosphoryl donors. Relative rates of MK activity when ATP, GTP, TTP and CTP were used as phosphoryl donors were 100, 15, 11 and 5 percent respectively (Fig. 23).

\subsubsection{Kinetic properties of AaMK}

Kinetic constants were measured for the purified recombinant AaMK using the enzyme-coupled assay. The AaMK had a $V_{\max }$ of $37 \mu \mathrm{mol} / \mathrm{min} / \mathrm{mg}$. The $K_{m s}$ (affinities) for MA and ATP were $90 \pm 18 \mu \mathrm{M}$ and $140 \pm 28 \mu \mathrm{M}$ respectively. The $K_{m}$ of the MK for MA was comparable to those previously described in archaea, bacteria and eukaryotes (Table 3). On the other hand, the $K_{m}$ for ATP varies greatly in different organisms.

\subsubsection{Feedback inhibition of AaMK}

AaMK activity was strongly inhibited by long chain isoprenoids, including GGPP, FPP and GPP with $K_{i}$ values of $0.55 \pm 0.28 \mu \mathrm{M}, 0.44 \pm 0.2 \mu \mathrm{M}$ and $0.93 \pm 0.19 \mu \mathrm{M}$ respectively (Fig. 24). Short chain isoprenoids, such as DMAPP and IPP inhibited only in the micromolar range, with a $K_{i}$ value greater than $10 \mu \mathrm{M}$; while $6 \mathrm{C}$ compounds, such as PM and DPM did not inhibit AaMK activity. Similar feedback inhibitions were described for other eukaryotic MKs (Table 4). 


\subsubsection{Inhibition of MK activity in crude extract (CE) of mosquitoes by long chain pyrophosphates}

The activity of MK in crude extracts from thoraxes dissected from sugar-feed (SF) mosquitoes $24 \mathrm{~h}$ after adult eclosion was measured using the enzyme-coupled assay. Addition of $100 \mu \mathrm{M}$ FPP decreased the kinase activity by $25 \%$ (Fig 25A). The optimal conditions for catalysis of three ATP-dependent enzymes (MK, phosphomevalonate kinase and mevalonate diphosphate decarboxylase) are similar (Rivera-Perez et al., 2014); therefore we hypothesized that in our in vitro assays the catalytic reactions continued from mevalonate (MA) to isopentenyl pyrophosphate (IPP) via phosphomevalonate (PM) and diphosphomevalonate (DPM). That prevented us from detecting changes in the pools of PM and DPM by HPLC; so we measured the changes in the pools of IPP as a proxy for MK activity (Fig. 25). In the presence of $100 \mu \mathrm{M}$ FPP we found a significant decrease in the pool of IPP, confirming the inhibitory effect of FPP on MK activity (Fig 25B).

\subsection{Discussion}

\subsubsection{Structural and biochemical characterization of AaMK}

We have biochemically characterized an A. aegypti MK involved in the synthesis of JH in the CA. An expressed sequence tag (EST) encoding AaMK was obtained from an A. aegypti corpora-allata + corpora cardiaca library, constructed and sequenced as previously described (Noriega et al., 2006). Analysis of the AaMK sequence revealed the features of a typical GHMP kinase protein. AaMK exhibits a compact globular $\alpha / \beta$ structure similar to that described for Rattus norvegicus MK (RnMK) (Fu et al., 2002). Analysis of the structure of $A a \mathrm{MK}$ revealed the presence of two large insertions in the Nterminal domain. The first insertion lies between $\beta 5$ and $\alpha 6$, and includes the disordered 
residues 71-110, located between the $\alpha 2$ and $\alpha 3$ (Fig. 18). The disordered residues in the sequence of $A a \mathrm{MK}$ are longer than in RnMK, which include residues 73-88 (Fu et al., 2002). The next insertion lies between $\alpha 5$ and $\alpha 6$ (Fig. 18). As these two insertions lie at the surface of the molecule, they are probably not involved in the catalytic function of the enzyme. However, these insertions must play a role in the stability of the MK protein (Fu et al., 2002).

The crystal structure of Methanococcus jannaschii MK (MjMK) revealed the existence of a disulfide bridge by $\mathrm{Cys}_{107}$ and $\mathrm{Cys}_{281}$ (Yang et al., 2002). The crystal structures of RnMK (Fu et al., 2002) and Streptococcus pneumoniae MK (SpMK) (Andreassi et al., 2007) were also solved. Despite the similar overall structural folding in these three enzymes, the disulfide bridge was absent in these 2 MKs. The cysteine residues forming the disulfide bond of $M j \mathrm{MK}$ are in motif II and motif IV respectively (Chu et al., 2007). Sequence alignments of the seven insects MK revealed that there is no cysteine residue present in the motif II (Fig. 19); therefore insects MKs do not have a disulfide bridge. The overall polypeptide folding of MKs from insects and rat are also very similar. The catalytic base aspartate and lysine are also conserved in the structure of insects; hence it is reasonable to propose a catalytic base mechanism for insects as shown in figure 20.

We measured the activity of the recombinant AaMK (Fig. 22), and compared it with different recombinant enzymes from archaea, bacteria and eukaryotes. Although it seems that the essential cation in vivo is probably $\mathrm{Mg}^{2+}$, our investigation of recombinant AaMK suggest that $\mathrm{Mg}^{2+}$ can be partially replaced in vitro by other divalent cations such as $\mathrm{Mn}^{2+}$ and $\mathrm{Co}^{2+}$ (Fig. 23). Similarly, in the process of phosphorylation, although other 
nucleotide triphosphates including GTP, CTP and TTP can partially substitute for ATP as phosphoryl donors in vitro (Fig. 23), most likely ATP is the preferential in vivo phosphoryl donor. The results for the analysis of cofactor requirements, phosphoryl source and optimal $\mathrm{pH}$ of the $A a \mathrm{MK}$ were in agreement with those from other previously characterized MKs (Voynova et al., 2003; Hedl and Rodwell, 2003).

Our kinetic studies revealed that the $A a \mathrm{MK} \mathrm{V}_{\max }$ for the formation of PM was comparable to that described for other MK's, ranging from 12 to $50 \mu \mathrm{mol} \mathrm{min}{ }^{-1} \mathrm{mg}^{-1}$ (Table 3). The AaMK Michaelis-Menten constants for mevalonate $\left(K_{M \mathrm{MA}}\right)$ and ATT ( $K_{M}$ ATP) were in the range of those previously described for other MKs: $K_{M \mathrm{MA}}$ are in a range of 19 to $236 \mu \mathrm{M}$; while $K_{M}$ ATP varied between 74 to $1180 \mu \mathrm{M}$ (Table 3).

It has been shown that the two phosphate groups of FPP compete for the binding of the phosphoryl groups of ATP; but large contributions to the inhibitor affinity are derived from binding interactions for the farnesyl moieties (Fu et al., 2008). The structure of animal MKs display an extra region of 25 to 50 amino acids between motif I and motif II, which are absent in bacterial and archaeal MKs. It constitutes part of the FPP binding pocket, thereby facilitating the isoprenoid binding in animal MKs (Fu et al., 2008). On the basis of the structure, kinetics and inhibition profile of the insect MKs, we could classify them into the same class of other animal MKs.

AaMK was also found to be inhibited by isoprenoids; with the pattern of this inhibition resembling well those of other eukaryotic MKs (Table 4). Inhibition of the activity of MKs by isoprenoids is competitive with respect to ATP (Fu et al., 2008), and the eukaryotic enzymes are 1000 fold more sensitive to the inhibition by isoprenoids than bacterial and archaeal enzymes (Table 4); even if their $K_{m}$ values differ by only 2-3 fold 
(Table 3). The MK activity in thoraces extracts of sugar-feed (SF) mosquitoes was significantly reduced in the presence of FPP, confirming the results obtained in the studies performed with the recombinant enzyme.

\subsubsection{Role of mevalonate kinase on JH synthesis in mosquitoes}

Mevalonate kinase is a key enzyme of the MVP and therefore for the synthesis of JH (Goodman and Cusson, 2012). AaMK is expressed in most adult female mosquito tissues (Fig. 26) (Nouzova et al., 2011); suggesting that $A a \mathrm{MK}$ is involved in many metabolic pathways. In the CA, there is a good correlation between AaMK mRNA expression and JH synthesis (Fig. 27) (Nouzova et al., 2011; Rivera-Perez et al., 2014). $\mathrm{JH}$ synthesis is suppressed during pupae development, and therefore AaMK mRNA levels are very low in the CA of early pupae. In adult females, CA AaMK transcript levels and $\mathrm{JH}$ synthesis reached maximum values during the first day after eclosion (Fig. 27A). In addition, changes in AaMK transcripts in the CA of sugar-fed and blood-fed female mosquitoes were also in agreement with the changes in JH synthesis (Fig. 27A). In larvae, pupae and adult female Bombyx mori the CA also displayed a good correlation between JH biosynthesis and expression of MK (Kinjoh et al., 2007). Likewise changes in AaMK enzymatic activity correlated well with rates of JH biosynthesis (Fig. 27B). The highest MK enzymatic activity was found in highly active glands (12h and 24h sugar-fed females), and the lowest enzymatic activity was found in the suppressed gland of bloodfed mosquitoes (Fig. 27B).

\subsection{Conclusions}

We have completed the first functional and molecular characterization of a mevalonate kinase involved in the production of JH in the CA of insects. AaMK is a class 
I MK that has the typical structure and functional features of other members of the GHMP kinase family. Changes in MK mRNA levels and enzymatic activity in the CA of pupa and adult female mosquitoes corresponded well with changes in $\mathrm{JH}$ synthesis, suggesting that $A a \mathrm{MK}$ transcript and activity fluctuations are at least partially responsible for the dynamic changes of $\mathrm{JH}$ biosynthesis during the gonotrophic cycle of female mosquitoes. The activity of AaMK was strongly inhibited in vitro and in vivo by isoprenoids such as GPP and FPP. Further studies are necessary to determine if the inhibition of MK activity by downstream metabolites might be important to regulate $\mathrm{JH}$ synthesis in the CA of insects. 


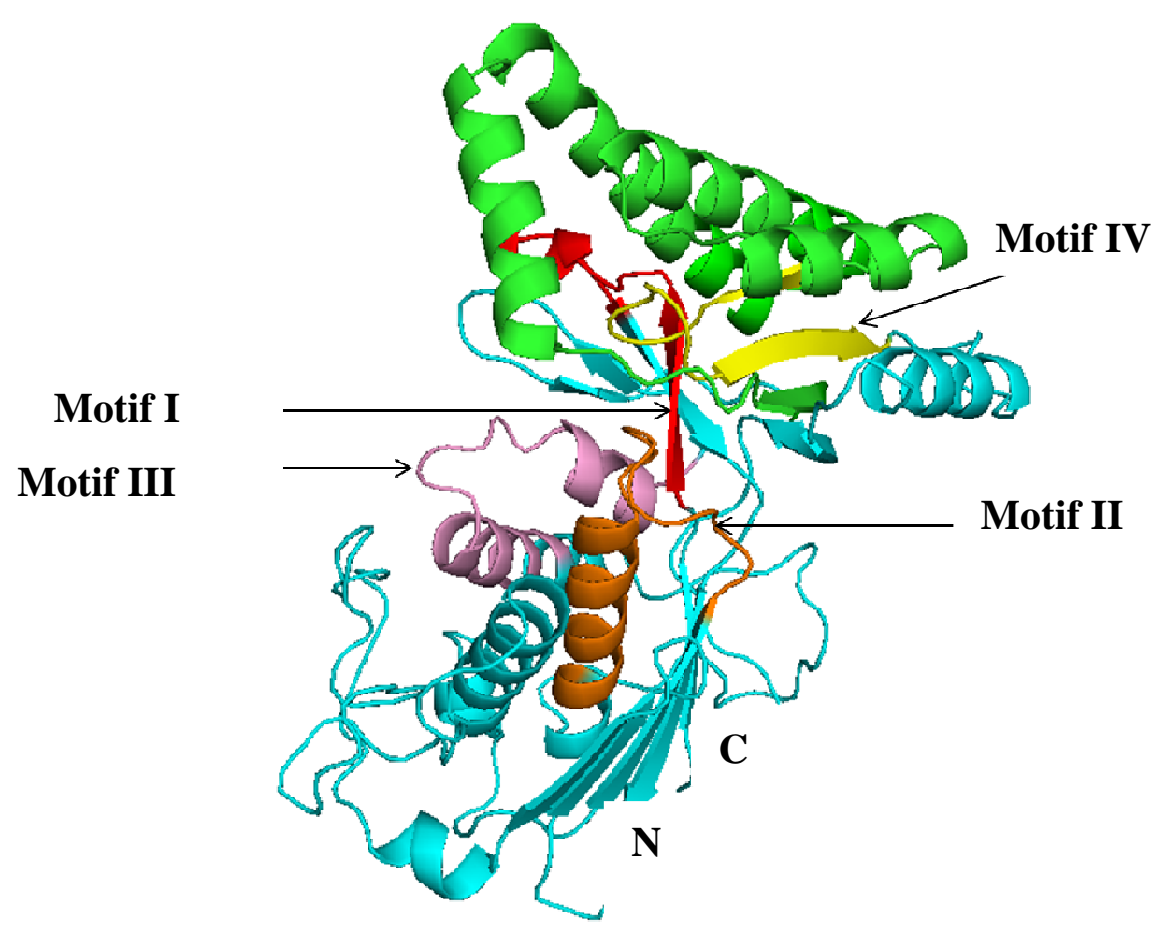

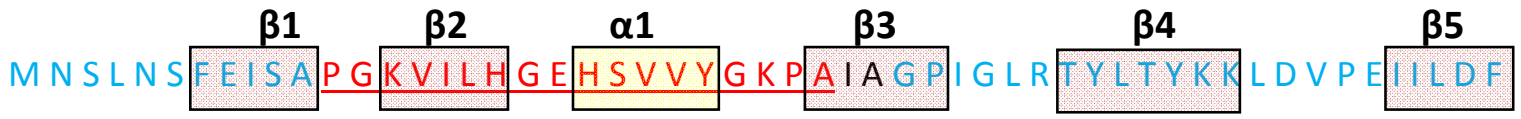
$\beta 6 \quad \alpha 2$

A S I P FTSKSL LSFNQF LQQHNCHSDMQPLEFLSKLRTSEEFPFAQYVSPQP

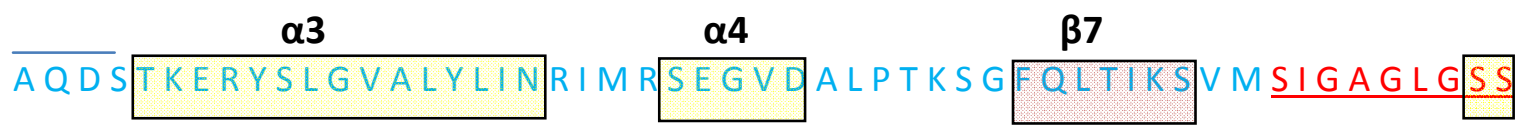

$\alpha 5 \quad \alpha 6$

AGYGVCVSAGAYVITKLAKGELTVDNALNYSFQGNEPEVLKKISQWAFD $\underline{S E}$
$\alpha 7$
$\beta 8 \quad \beta 9$
$\alpha 8$

LVMHERPSG IDNTICTYG N LIKFRKGEP FESLKLRQQINILIVDTKVSRTTSKL

$\begin{array}{lll}\alpha 9 & \alpha 10 & \alpha 11\end{array}$

VANVAALKNKH KMM I SILDAMGHLVDDAVELLEDERDQFEALRTLVAVNN

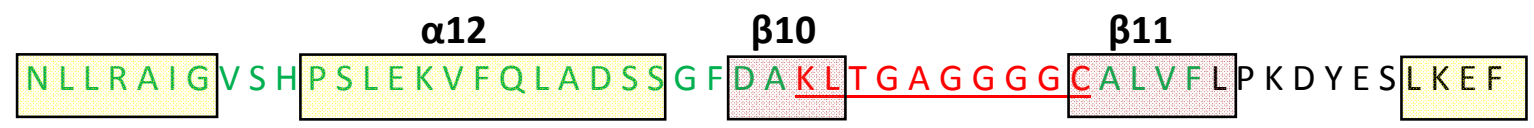

$\alpha 13 \quad \beta 12$

GVLTGSLTEA GFSWMATTIGGSGVEFKAIE 
Figure 18: Homology model of the overall fold of $A a \mathrm{MK}$ and its amino acid sequence. (A) Three dimensional structure of $A a \mathrm{MK}$. The N-terminal is shown in cyan, C-terminal is green, and motifs are indicated by colors; motif I (red), motif II (orange), motif III (pink) and motif IV (yellow). This structure was constructed by PyMOL using the Human MK (PDB: 2r3v.3) as template. (B) N-terminal amino acids are shown in black and $\mathrm{C}$ terminal with green color. Helices are numbered $\alpha 1$ through $\alpha 13$ and shown inside yellow box, $\beta$-strand are numbered $\beta 1$ through $\beta 12$ and shown inside red box. Motifs I-IV are shown in red underlined color. Two large insertions (i.e. residues 71-110 and residues 175-194) not involved in the catalytic function of AaMK are shown with blue line above them. 
AaMK 12 PGKVILHGEHSVVYGKPA 29151 SIGAGLGSSAG 161209 SELVMHERPSGIDN 222343 KLTGAGGGGC 352 CqMK 11 PGKVILHGEHSVVYGKPA 28148 SIGAGLGSSAG 158201 SEIVMHERPSGIDN 214335 KLTGAGGGGC 344 AgMK 14 PGKVILHGEHSVVYGHPA 31 160 SIGAGLGSSAS 170210 SEIIMHVKPSGIDN 223348 KLTGAGGGGC 359 DmMK 9 PGKVILHGEHAVVYHRPA 26148 TVGAGLGSSAS 158195 SERVNHGTPSGLDN 208335 KLTGAGAGGY 344 BMMK 16 PGKVILHGEHSVVYGKTA 33161 TIGAGTGSSAS 171219 CEKIMHGTPSGIDN 232362 KLTGAGGGGY 371 DPMK 11 PGKVILHGEHSVLYGEIA 28150 TIGAGTGSSAS 160199 SEKIMHGTPSGIDN 212341 KLTGAGGGGH 350 AmMK 9 PGKVILFGEHAVVYGKTA 26138 AINSGLGSSAS 148186 CERIMHGNPSGIDN 199332 KLTGAGGGGH 343 ApMK 12 PGKIILFGEHSVVYGKPA 29146 KLGAGTGSSAS 156223 AENFIHTKASGLDN 236365 KLTGAGMGGY 374 RnMK 11 PGKVILHGEHAVVHGKVA 28138 PPGAGLGSSAA 148192 GERMIHGNPSGVDN 205330 KLTGAGGGGC 339 $* * *: * * * * *: *::$ : $*$ $:^{*} * * *$. *.*.**:**

$\star * * * * * * *$

Figure 19: Amino acid sequence alignment of conserved motifs of MK from A. aegypti, compared with seven different insects and rat orthologs. Amino acid sequence alignment of MK motifs from Aedes aegypti (AaMK), Culex quinquefasciatus (CqMK), Anopheles gambiae (AmMK), Drosophila melanogaster (DmMK), Bombyx mori (BmMK), Danaus plexippus (DpMK), Apis mellifera (AmMK), Acyrthosiphon pisum (ApMK) and Rattus norvegicus (RnMK). Catalytic aspartate is shown in red and lysine in blue color. The suggested functions for the motifs as according to (Houten et al., 2000) are Motif I has shown to be involved in targeting MK to peroxisomes and also in the stabilization of ATP binding and protein tertiary and quaternary structure. Motif II has an ATP binding site and also its high hydrophobicity makes it a good candidate for isoprenoid binding site. Motif III has function in the activation of the enzyme. Motif IV is involved in the stabilization of the mevalonate binding. Accession numbers: AaMK (AAEL006435), CqMK (EDS42994.1), AgMK (EAA14782.5), DmMK (AGB93455.1), BmMK (NP_001093299.1), DpMK (EHJ79258), AmMK $\quad\left(\mathrm{XP}_{-} 006558673.1\right), \quad$ ApMK (XP_001942835) and RnMK (NP_112325.1) 


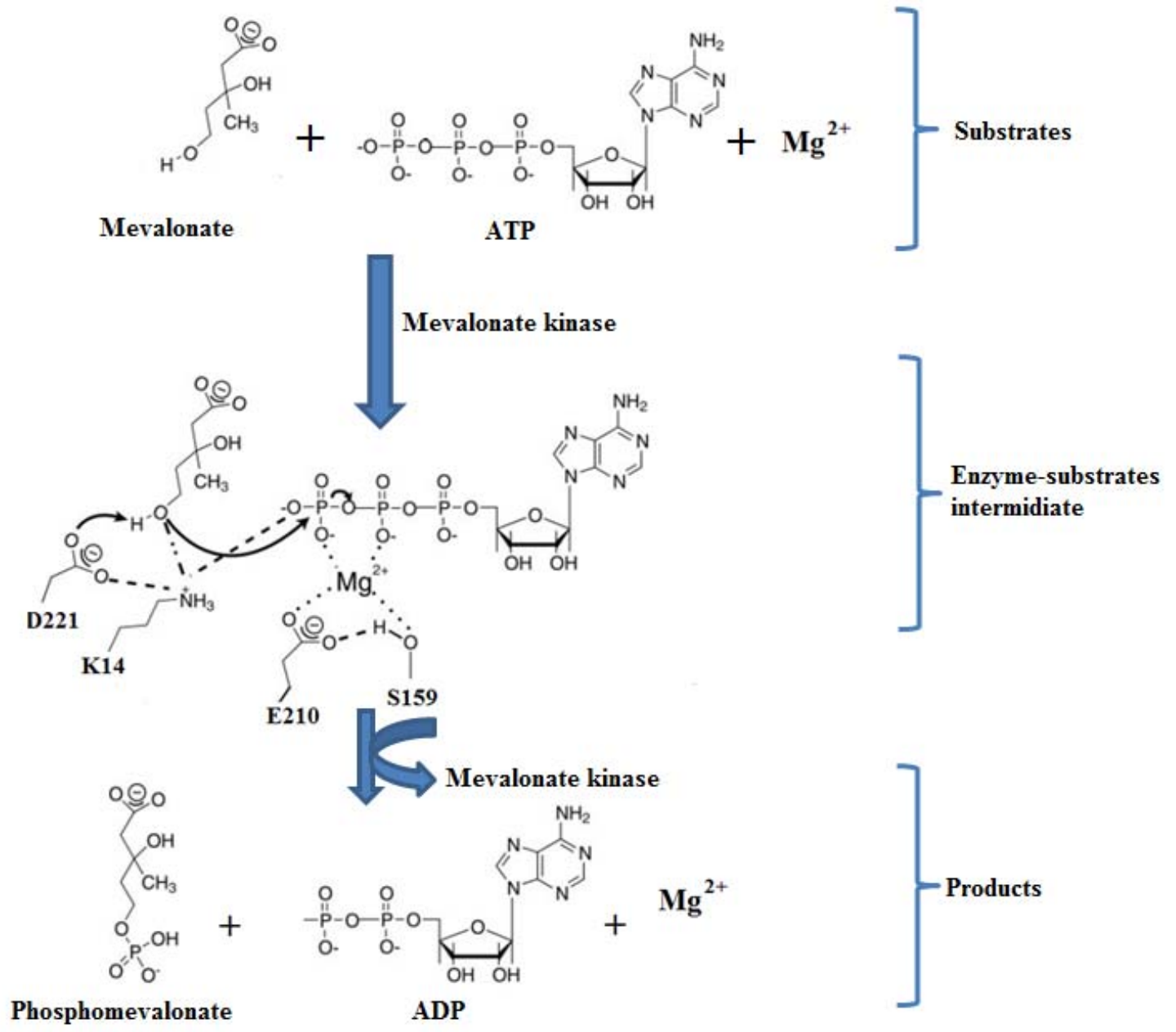

Figure 20: Scheme of catalytic mechanism of AaMK. This outline for Aedes aegypti MK $(A a \mathrm{MK})$ reaction has been inspired from previously postulated catalytic base mechanism for the rat, Rattus norvegicus MK (RnMK) (Fu et al., 2002). Asp $^{221}$ makes a salt bridge with Lys ${ }^{14}$; the penta-coordinated $\gamma$-phosphate transition state is stabilized by the $\mathrm{Mg}^{2+}$, $\mathrm{Glu}^{210}, \mathrm{Ser}^{159}$ and $\mathrm{Lys}^{14}$. Asp ${ }^{204}$ acts as a general base, abstracting a proton from the hydroxyl group in MA. This converts MA into an excellent nucleophile, which then attacks the $\gamma$-phosphorus of ATP. Lys ${ }^{13}$ is believed to maintain the aspartate residue in the deprotonated state and lower its $\mathrm{p} K_{a}$ to facilitate the proton transfer. 
A)

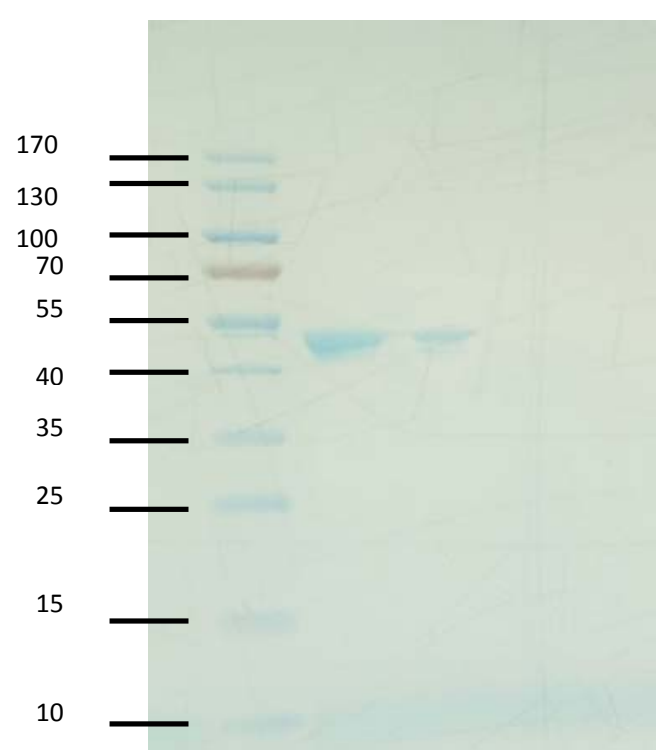

B)

MK

45.4 KDa

Figure 21: SDS PAGE (A) and Western blot (B) analysis of the recombinant mosquito AaMK. Lane contents of the gel were: 1, molecular weight standard; 2 and 3, AaMK. Molecular weight of protein standards are depicted on the $\mathrm{Y}$ axis. 


\section{A)}

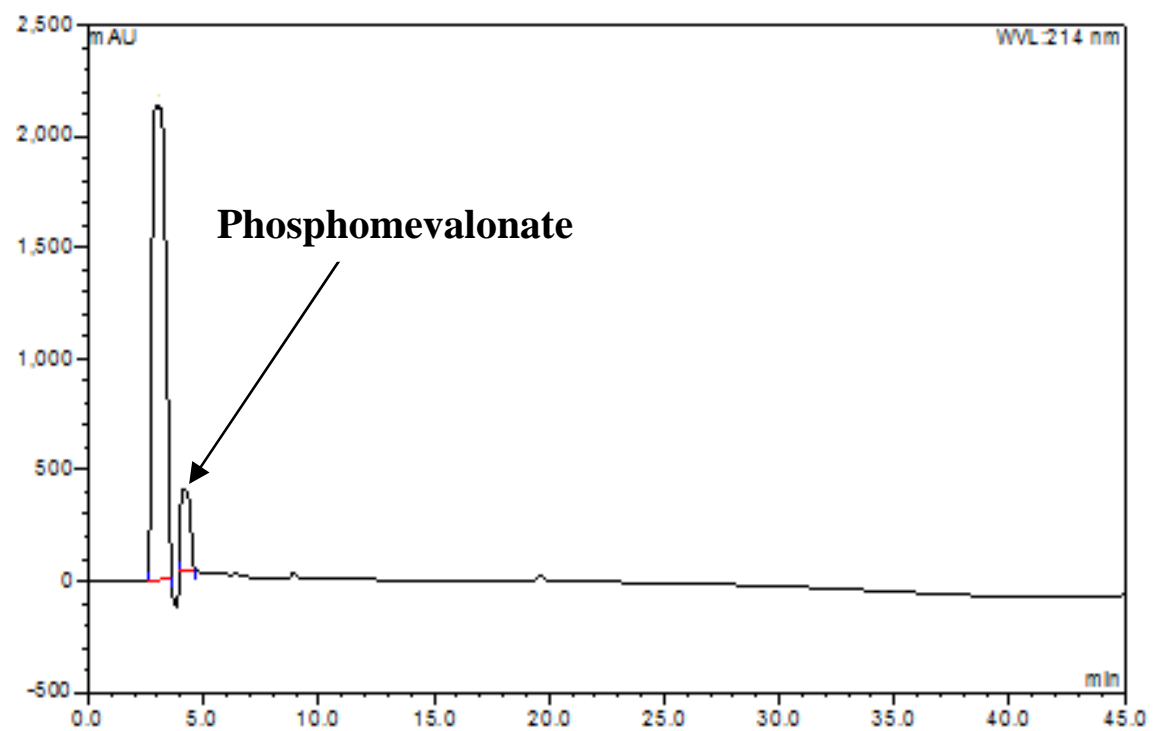

B)

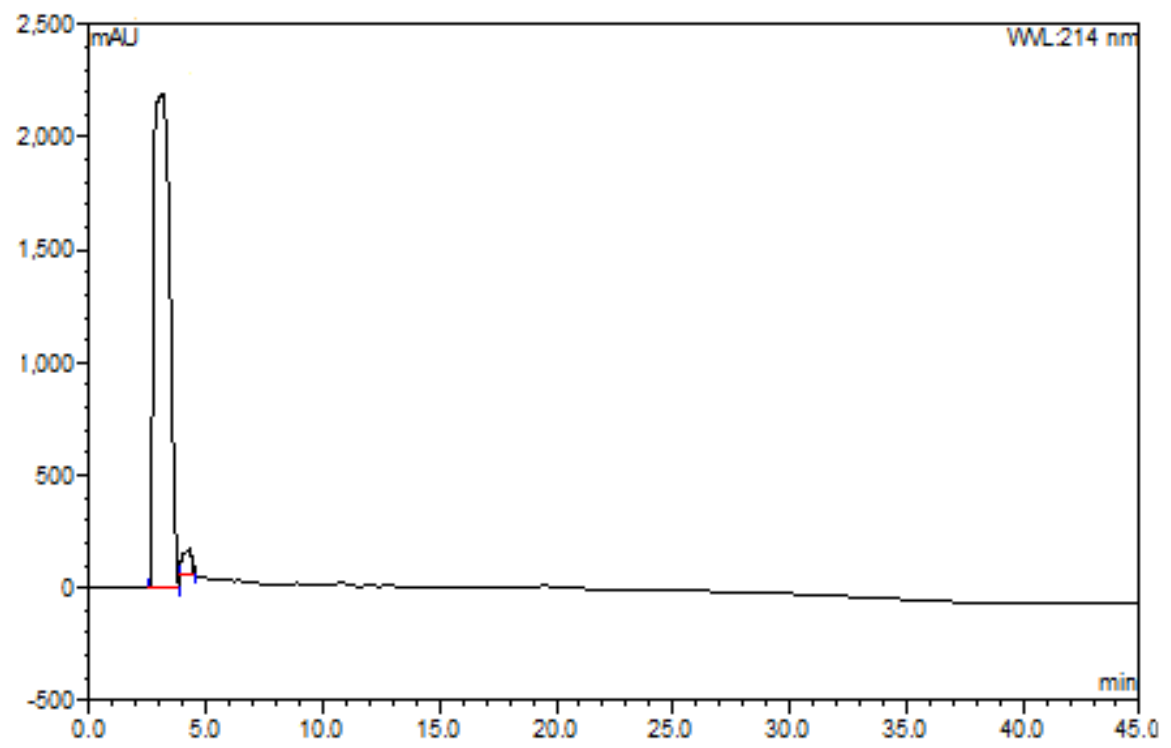

Figure 22: Chromatogram of a reverse phase-HPLC analysis showing the production of PM from MA by AaMK. (A) Mevalonate kinase reaction in which $100 \mu \mathrm{M}$ MA and 100 $\mu \mathrm{M}$ ATP were incubated with AaMK in reaction buffer for $1 \mathrm{~h}$ at $30^{\circ} \mathrm{C}$. Arrow indicates PM. (B) Negative control in which $100 \mu \mathrm{M}$ MA and $100 \mu \mathrm{M}$ ATP were incubated in reaction buffer without adding enzyme for $1 \mathrm{~h}$ at $30^{\circ} \mathrm{C}$. 
A)

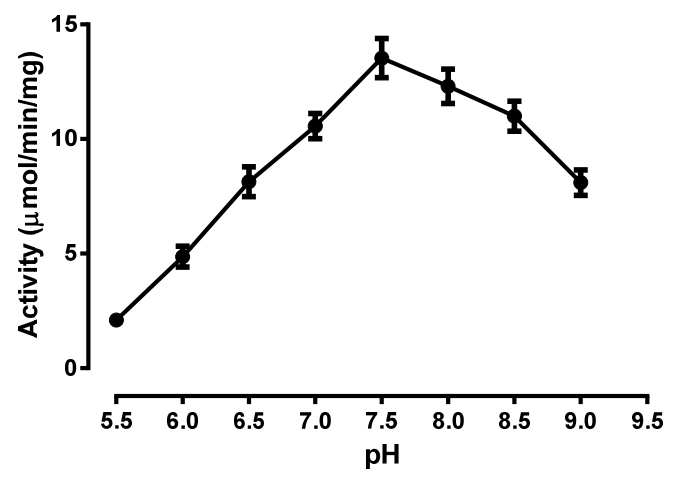

C)

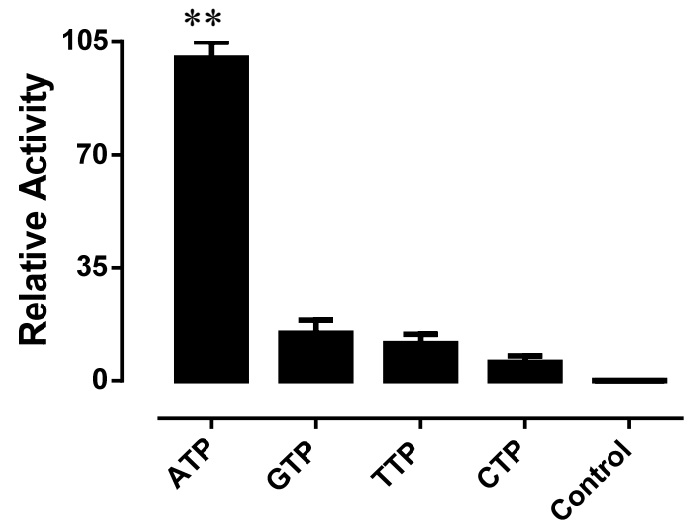

B)

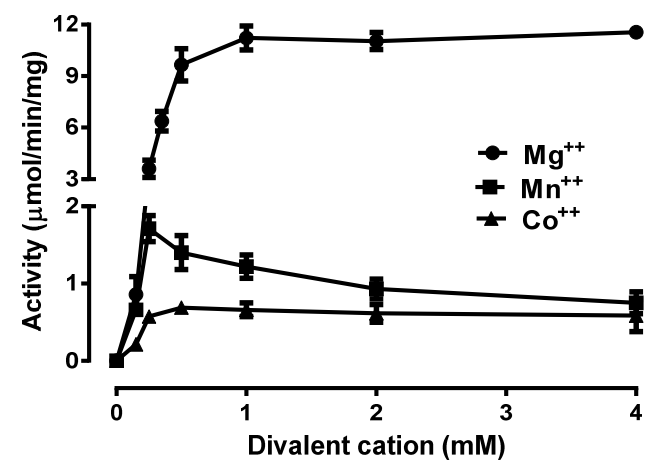

Figure 23: AaMK pH curve, effect of metal ions and specificity of the phosphoryl donor. Kinase activity was measured by enzyme coupled spectrophotometric assay; ATP and MA were held constant and data were normalized to the maximum observed reaction velocities. To ensure MK was the rate-limiting enzyme, when necessary results were verified with the following conditions: doubling the amount of MK added doubled the observed rate, doubling the amount of PK and LDH did not affect the observed rate, and doubling the PEP concentration did not affect the observed rate. (A) $\mathrm{pH}$ curve. Two different buffers were used: MES at $\mathrm{pH} 5.5$ to 7 and Tris- $\mathrm{HCl}$ at $\mathrm{pH} 7$ to 9. (B) Effect of metal ions. Different metal cofactors $\mathrm{Mg}^{2+}, \mathrm{Mn}^{2+}$ and $\mathrm{Co}^{2+}$ were assayed for the $\mathrm{MK}$ activity. (C) Specificity of the phosphoryl donor. Each value represents the means \pm S.E. of three replicate assays. Relative activity is defined as a percentage of the highest value recorded. 


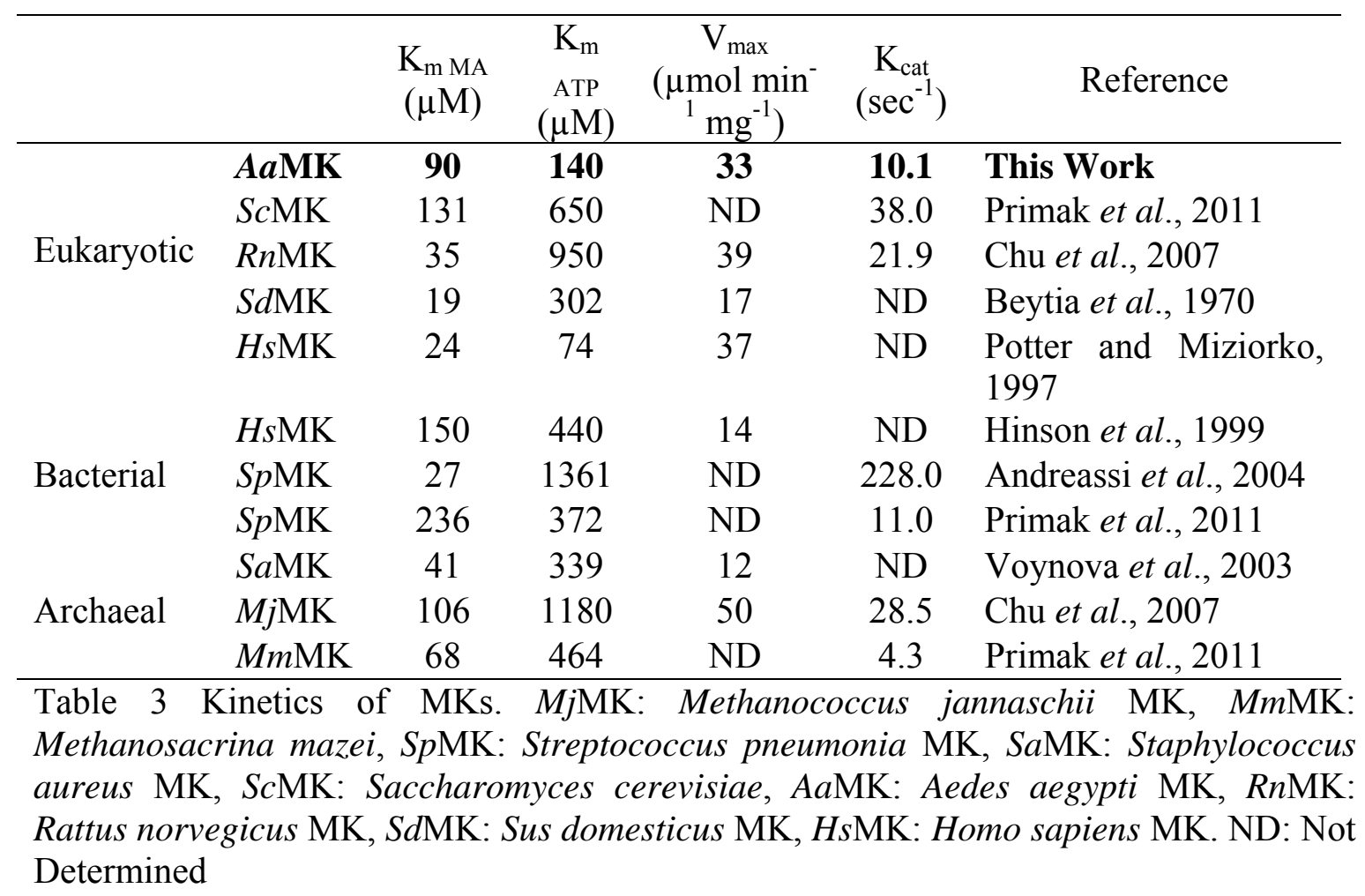




\section{A)}

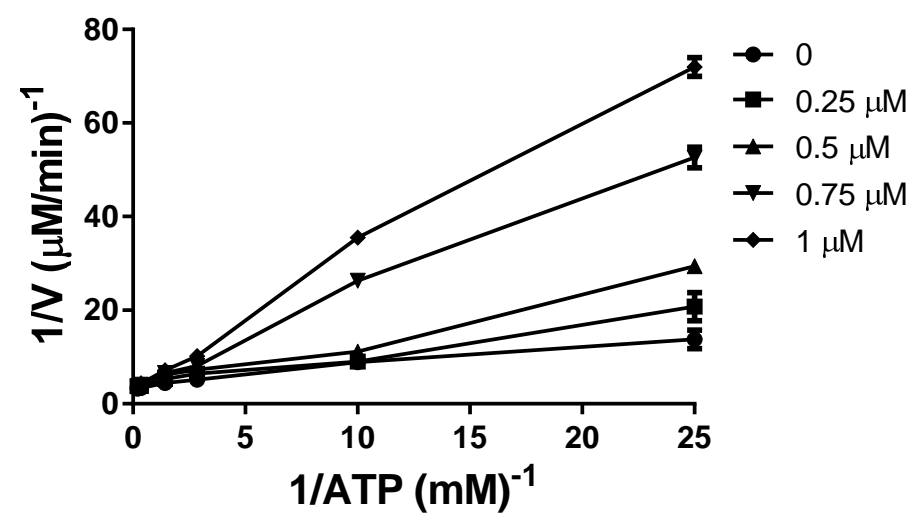

B)

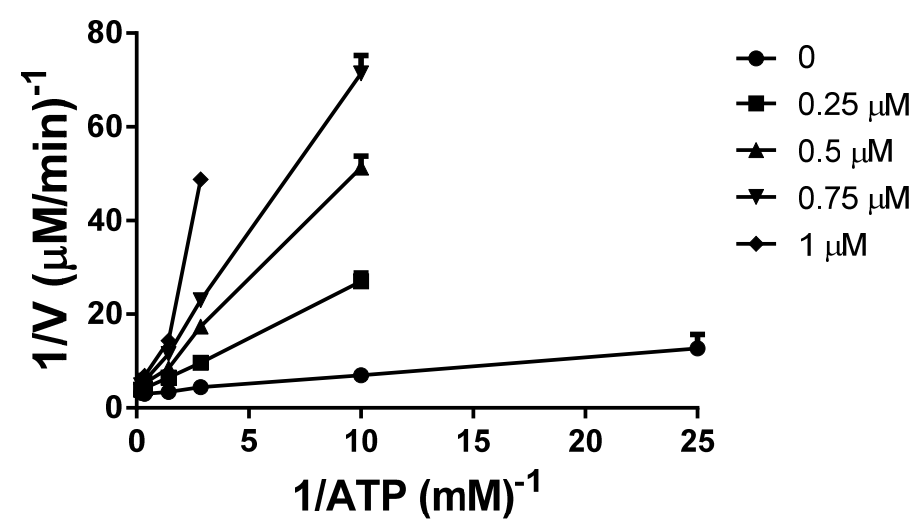

C)

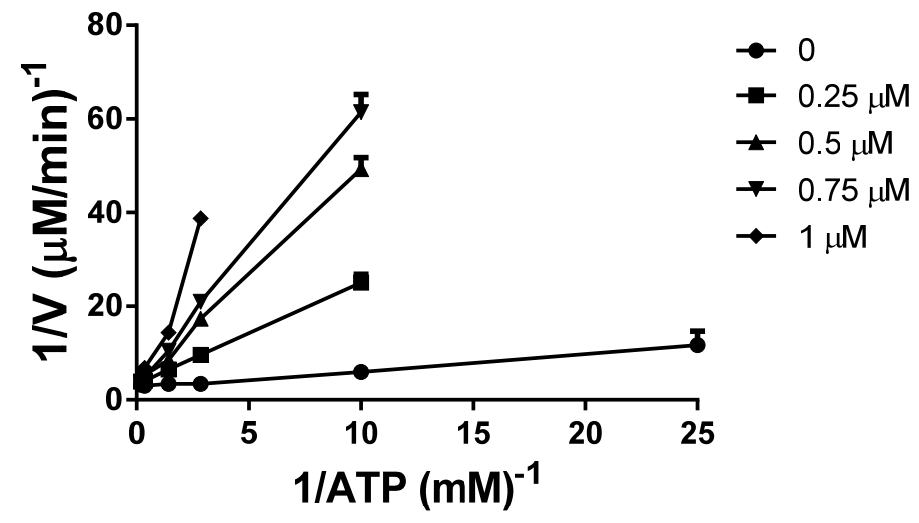

Figure 24: Inhibition of AaMK activity by GPP (A), FPP (B) and GGPP (C). The rate of MK activity was measured at different ATP concentrations, without inhibitor and with several fixed concentration of inhibitor (indicated in bracket). Secondary plots of slope versus inhibitor concentration indicated that the Ki values for GPP, FPP and GGPP were respectively $0.93 \pm 0.19 \mu \mathrm{M}, 0.44 \pm 0.2 \mu \mathrm{M}$ and $0.55 \pm 0.28 \mu \mathrm{M}$. 


\begin{tabular}{|c|c|c|c|c|c|}
\hline & $\begin{array}{l}\text { Recombinant } \\
\text { Enzyme }\end{array}$ & $\begin{array}{c}\text { Ki DPM } \\
(\mu \mathrm{M})\end{array}$ & $\begin{array}{c}\text { Ki } \\
\text { GPP } \\
(\mu M)\end{array}$ & $\begin{array}{c}\text { Ki } \\
\text { FPP } \\
(\mu M)\end{array}$ & References \\
\hline \multirow{5}{*}{ Eukaryotic } & AaMK & NI & 0.93 & 0.54 & This Work \\
\hline & ScMK & NI & 0.25 & 0.13 & Primak et al., 2011 \\
\hline & RnMK & NI & ND & 2.5 & Tanaka et al., 1990 \\
\hline & PigMK & NI & 2 & 2 & Beytia et al., 1970 \\
\hline & HsMK & NI & 0.116 & 0.104 & Hinson et al., 1999 \\
\hline \multirow[t]{3}{*}{ Bacterial } & SpMK & 0.63 & NI & NI & Andreassi et al., 2004 \\
\hline & SpMK & Inhibition & NI & NI & Primak et al., 2011 \\
\hline & SaMK & NI & ND & $>10$ & Voynova et al., 2003 \\
\hline \multirow[t]{2}{*}{ Archaeal } & MjMK & NI & $>10$ & $>10$ & Huang et al., 1999 \\
\hline & MmMK & NI & NI & NI & Primak et al., 2011 \\
\hline
\end{tabular}

Table 4: Feedback Inhibition of MK using short (DPM, 6C) and long chain isoprenoids (GPP, 10 C; FPP, 15C). MjMK: Methanococcus jannaschii MK, MmMK: Methanosacrina mazei, SpMK: Streptococcus pneumonia MK, SaMK: Staphylococcus aureus MK, ScMK: Saccharomyces cerevisiae, AaMK: Aedes aegypti MK, RnMK: Rattus norvegicus MK, SdMK: Sus domesticus MK, HsMK: Homo sapiens MK. NI: No Inhibition ND: Not Determined 
A)

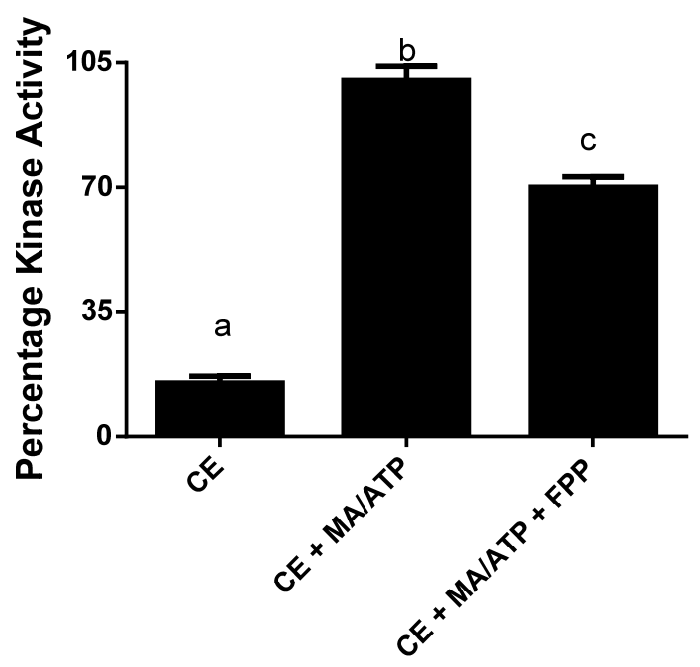

B)

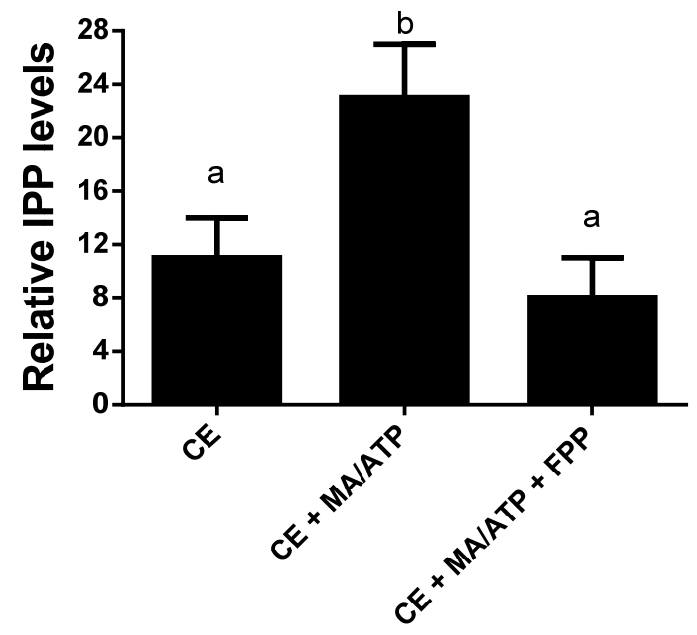

Figure 25: Inhibition of mevalonate pathway and $A a \mathrm{MK}$ activity by long chain pyrophosphates. (A) Inhibition of the mevalonate pathway kinase activity by FPP: $5 \mu \mathrm{g}$ protein from thorax extracts dissected from sugar-fed females $24 \mathrm{~h}$ after emergence were incubated in presence and absence of $100 \mu \mathrm{M}$ FPP in Tris-HCl buffer, $\mathrm{pH} 7.5$ containing $5 \mathrm{mM} \mathrm{MgCl}_{2}$ and $1 \mathrm{mM}$ DTT. Kinase activities ware measured by enzyme coupled assay. (B) Inhibition of the AaMK activity by FPP: $5 \mu$ g protein from thorax extracts dissected from sugar-fed females $24 \mathrm{~h}$ after emergence were incubated in absence and presence of $100 \mu \mathrm{M}$ FPP in Tris-HCl buffer, $\mathrm{pH} 7.5$ containing $5 \mathrm{mM} \mathrm{MgCl}_{2}, 1 \mathrm{mM}$ DTT, $500 \mu \mathrm{M}$ MA \& $500 \mu \mathrm{M}$ ATP. CE represents the endogenous levels of IPP. Bars represent the means \pm SD of three replicates of extracts from groups of 3 thoraxes. Different letters above the columns indicate significant differences among treatments (one way ANOVA $\mathrm{p}<0.05$, with Tukey's test of multiple comparisons). 


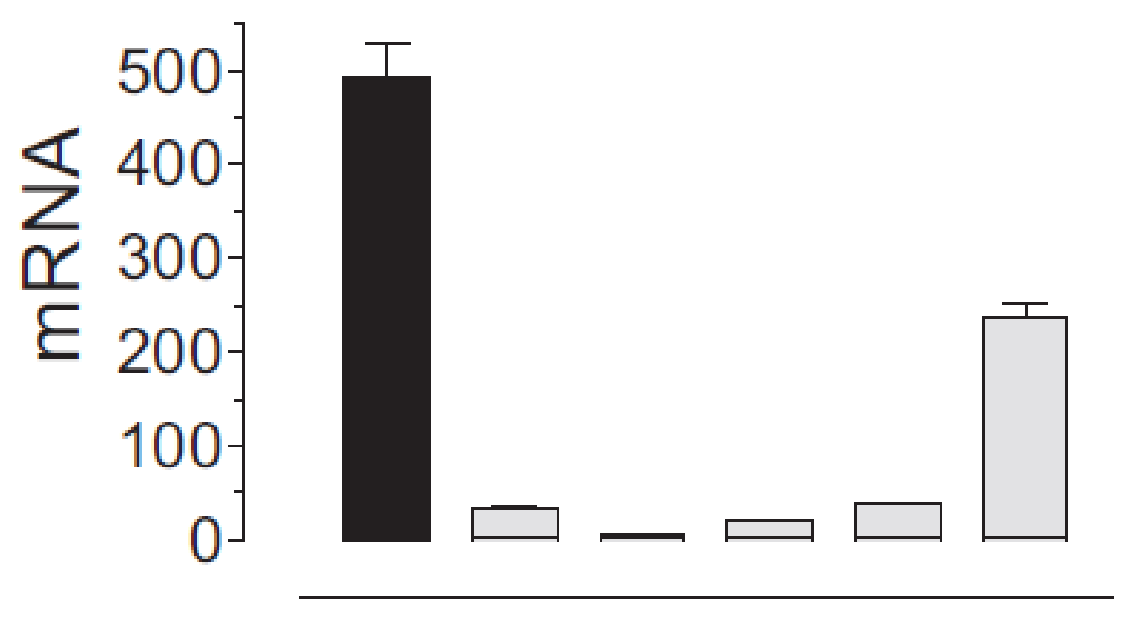

\section{CA BR FB MG MT OV}

Fig. 26: Tissue specific expression of AaMK. All tissues were dissected from 3-day-old sugar-fed females. BR: brain; CA: corpora allata; BR: brain; FB: fat body; MG: midgut; MT: malpighian tubules; OV: ovaries. Each value represents the means \pm S.E.M of two independent biological replicates of 10-20 tissue samples evaluated in triplicate. AaFPPase mRNAs are expressed as copy number of mRNA/10,000 copies of rpL32 mRNA (Nouzova et al., 2011). 


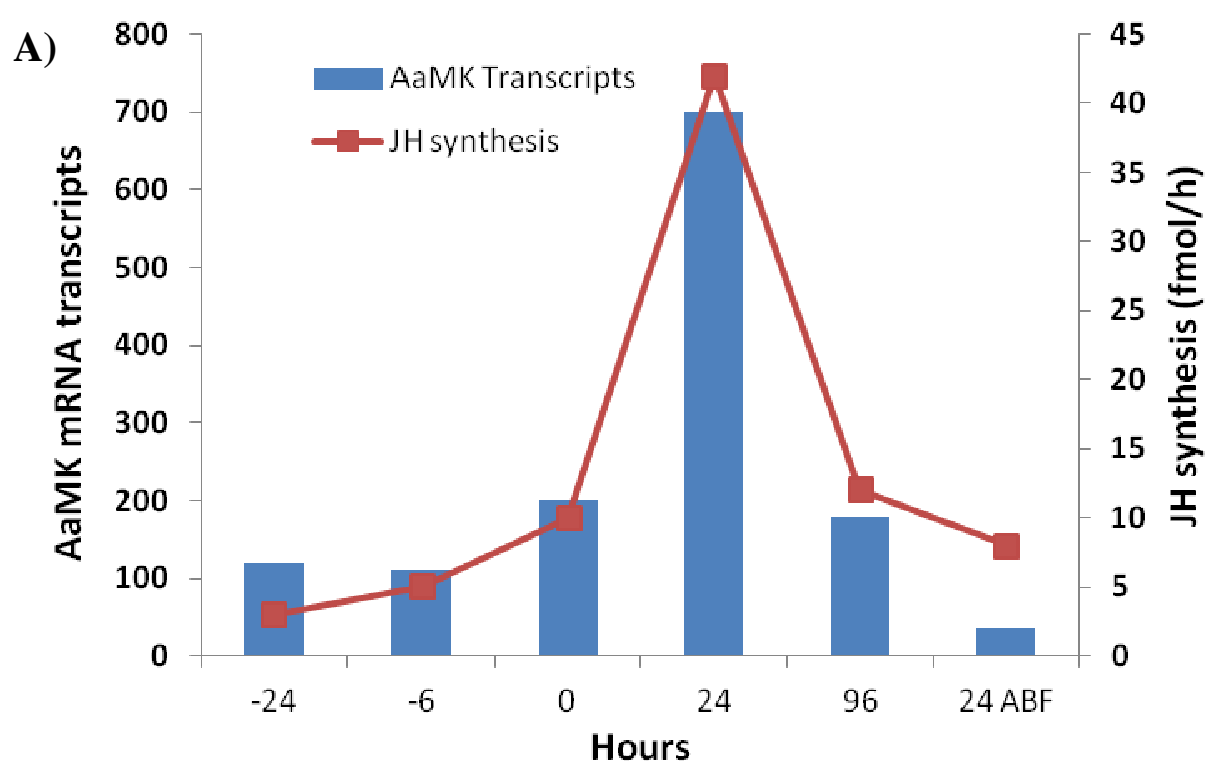

B)

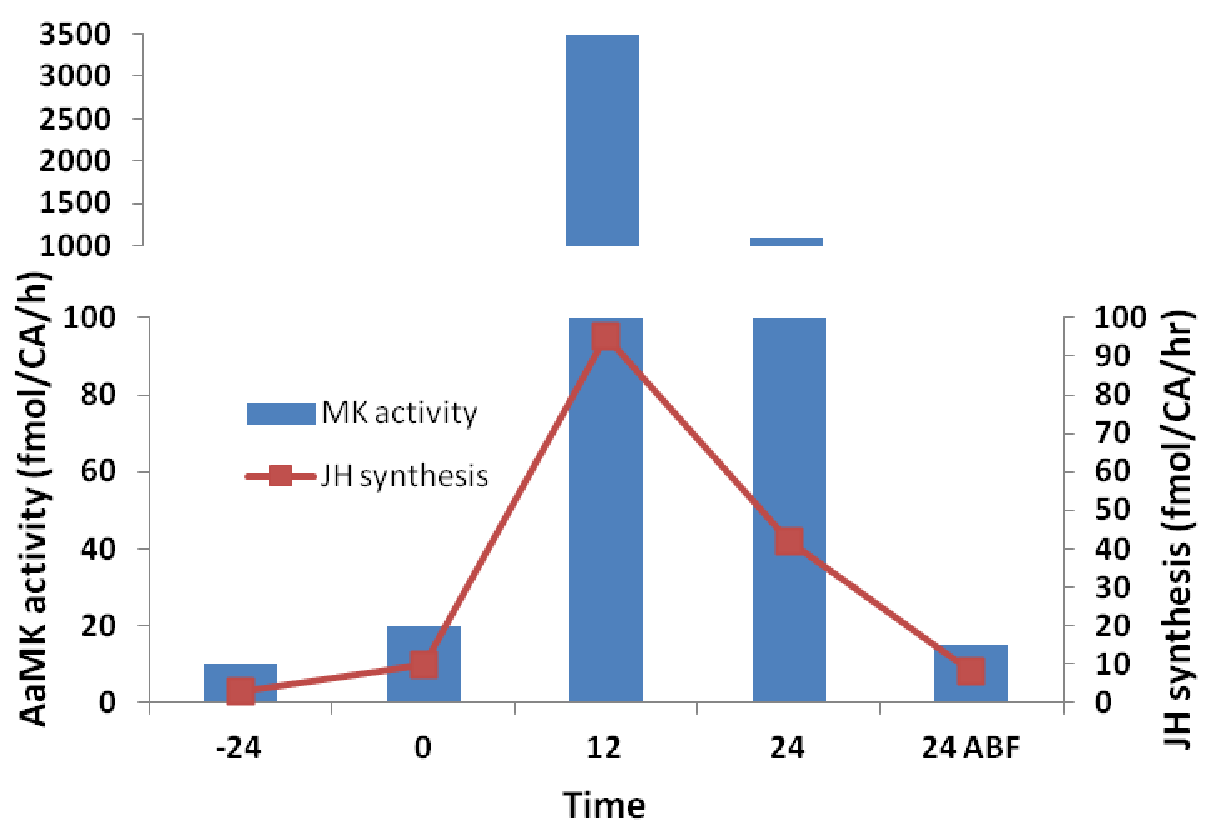

Fig. 27: Transcript levels (A) and enzyme activity (B) of the AaMK in the developmental stage of female A. aegypti. Each data point is the means \pm S.E.M. of three independent biological replicates of $20 \mathrm{CA}$ evaluated in triplicate. AaMK mRNAs are expressed as copy number of mRNA/10,000 copies of rpL32 mRNA (Modified from Nouzova et al., 2011 \& Rivera-Perez et al., 2014). 


\subsection{References}

1. Amdur BH, Rilling H, Bloch K (1957) The enzymatic conversation of mevalonic acid to squalene. J Am Chem Soc 79:2646.

2. Andreassi JL, Dabovic K, Leyh TS (2004) Streptococcus pneumonia isoprenoid biosynthesis is downregulated by diphosphomevalonate: an antimicrobial target. Biochemistry 43:16461-16466.

3. Andreassi JL, Bilder PW, Vetting MW, Roderick SL, Leyh TS (2007) Crystal structure of the Streptococcus pneumoniae mevalonate kinase in complex with diphosphomevalonate, Protein Sci 16:983-989.

4. Arnold K, Bordoli L, Kopp J, Schwede T (2006) The SWISS-MODEL Workspace: A web-based environment for protein structure homology modeling. Bioinformatics 22:195-201.

5. Belles X, Martin D, Piulachs MD (2005) The mevalonate pathway and the synthesis of juvenile hormone in insects. Ann Rev Entomol 50:181-199.

6. Beytia EJ, Dorsey KJ, Marr J, Cleland WW, Porter JW (1970) Purification and mechanism of action of hog liver mevalonate kinase. J Biol Chem 245:5450-5458.

7. Bork P, Sander C, Valencia A (1993) Convergent evolution of similar enzymatic function on different protein folds: the hexokinase, ribokinase, and galactokinase families of sugar kinases. Protein Sci 2: 31-40.

8. Cheek S, Zhang H, Grishin NV (2002) Sequence and structure classification of kinase. J Mol Biol 320:855-881.

9. Chu X, Yu W, Wu L, Liu X, Li N, Li D (2007) Effect of a disulfide bond on mevalonate kinase. Biochim Biophys Acta 1774:1571-1581.

10. Fu Z, Voynova NE, Herdendorf TJ, Miziorko HM, Kim JP (2008) Biochemical and structural basis for feedback inhibition of mevalonate kinase and isoprenoid metabolism. Biochemistry 47:3715-3724.

11. Fu Z,Wang M, Potter D, Miziorko HM, Kim JJ (2002) The structure of a binary complex between a mammalian mevalonate kinase and ATP: Insights into the reaction mechanism and human inherited disease. J Biol Chem 277:18134-18142.

12. Goodman WG, Cusson M (2012) The Juvenile Hormones. In Insect Endocrinology (Gilbert LI, ed), Elsevier, pp. 310-365. 
13. Hedl M, Rodwell VW (2003) Enterococcus faecalis mevalonate kinase. Protein Sci 13:687-693.

14. Hinson DD, Chambliss KL, Toth MJ, Tanaka RD, Gibson KM (1997) Posttranslational regulation of mevalonate kinase by intermediates of the cholesterol and nonsterol isoprene biosynthetic pathway. J Lipid Res 38:2216-2223.

15. Hoffman G, Gibson KM, Brandt IK, Bader PI, Wappner RS, and Sweetman L (1986) Mevalonic Aciduria: An Inborn Error of Cholesterol and Nonsterol Isoprene Biosynthesis. N Engl J Med 314:1610-1614.

16. Houten SM, Wanders RJ, Waterham HR (2000) Biochemical and genetic aspects of mevalonate kinase and its deficiency. Biochim Biophys Acta 1529:19-32.

17. Huang K, Scott AI, Bennett GN (1999) Overexpression, purification, and characterization of the thermo stable mevalonate kinase from Methanococcus jannaschii. Protein Expression Purif 17:33-40.

18. Huang M, Li X, Zou JW, Timson DJ (2013) Role of Arg228 in the phosphorylation of galactokinase: the mechanism of GHMP kinases by quantum mechanics/molecular mechanics studies. Biochemistry 52(28):4858-68.

19. Gray JC, Kekwick RGO (1972) The inhibition of plant mevalonate kinase preparations by prenyl pyrophosphates. Biochim. Biophys. Acta 279:290-296.

20. Kinjoh T, Kaneko Y, Itoyama K, Mita K, Hiruma K, Shinoda T (2007) Control of juvenile hormone biosynthesis in Bombyx mori: cloning of the enzymes in the mevalonate pathway and assessment of their developmental expression in the corpora allata. Insect Biochem Molec Biol 37:808-818.

21. Krishna SS, Zhou T, Daugherty M, Osterman A, Zhang H (2001) Structural basis for the catalysis and substrate specificity of homoserine kinase. Biochemistry 40:10810-10818.

22. Lawson D, Arensburger P, Atkinson P, Besansky NJ, Bruggner RV, Butler R et al (2009) VectorBase: a data resource for invertebrate vector genomics. Nucleic Acids Res 37:583-587.

23. Lombard J, Moreira D, (2010) Origins and early evolution of the mevalonate pathway of isoprenoid biosynthesis in the three domains of life. Mol Biol Evol 28:87-99. 
24. Noriega F, Ribeiro JMC, Koener JF, Valenzuela JG, Hernandez-Martinez S, Pham VM, Feyereisen R (2006) Comparative genomics of insect juvenile hormone biosynthesis. Insect Biochem. Mol Biol 36:366-374.

25. Noriega FG (2014) Juvenile Hormone Biosynthesis in Insects: What Is New, What Do We Know, and What Questions Remain? ISRN 2014:967361.

26. Nouzova M, Edwards MJ, Mayoral JG, Noriega FG, 2011 A coordinated expression of biosynthetic enzyme controls the flux of juvenile hormone precursors in the corpora allata of mosquitoes. Insect Biochem Mol Biol 9:660-669.

27. Nyati P, Nouzova M, Rivera-Perez C, Clifton ME, Mayoral JG Noriega FG (2013) Farnesyl phosphatase, a corpora allata enzyme involved in juvenile hormone synthesis in Aedes aegypti. PLoS ONE 8(8): e71967. doi:10.1371/journal.pone.0071967.

28. Oulmouden A, Karst F (1991) Nucleotide sequence of the ERG12 gene of Saccharomyces cerevisiae encoding mevalonate kinase.

29. Peter Y, Chou P, Gerald D (1974) Prediction of protein conformation Biochemistry 13:222-245.

30. Potter D, Miziorko HM (1997) Identification of catalytic residues in human mevalonate kinase J Biol Chem 272:25449-25454.

31. Potter D, Wojnar JM, Narasimhan C, Miziorko HM (1997) Identification and functional characterization of an active-site lysine in mevalonate kinase. J Biol Chem 272:5741-5746.

32. Primak YA, Du M, Miller MC, Wells DH, Nielsen AT, Weyler W, Beck ZQ Characterization of a Feedback-Resistant Mevalonate Kinase from the Archaeon Methanosarcina mazeiAppl Environ Microbiol 77(21):7772-8.

33. Rivera-Perez C, Nouzova M, Lamboglia I, Noriega FG (2014) Metabolic analysis reveals changes in the mevalonate and juvenile hormone synthesis pathways linked to the mosquito reproductive physiology. Insect Biochem Mol Biol 51:1-9.

34. Schwede T, Kopp J, Guex N, Peitsch MC (2003) SWISS-MODEL: an automated protein homology-modeling server. Nucleic Acids Res 31:3381-3385.

35. Tanaka RD, Lee LY, Schafer BL, Kratunis VJ, Mohler WA, Robinson GW, Mosley ST (1990) Molecular cloning of mevalonate kinase and regulation of its mRNA levels in rat liver. Proc Natl Acad Sci USA 87:2872-2876. 
36. Thoden JB, Holden HM (2005) The Molecular architecture of Human NAcetylgalactosamine kinase. J Biol Chem 280:32784-32791.

37. Voynova NE, Rios SE, Miziorko HM (2003) Staphylococcus aureus mevalonate kinase: isolation and characterization of an enzyme of the isoprenoid biosynthetic pathway. J Bacteriol 186:61-67.

38. Yang D, Shipman LW, Roessner CA, Scott AI, Sacchettini JC (2002) Structure of the Methanococcus jannaschii mevalonate kinase, a member of the GHMP kinase superfamily J Biol Chem 277:9462-9467. 


\section{Chapter 4: Characterization of additional corpora allata enzymes involved in juvenile hormone biosynthesis pathway}

\subsection{Abstract}

The present study describes the partial characterization of three additional CA enzymes involved in JH synthesis: HMG-CoA synthase (HMGS), phosphomevalonate kinase (PMK) and FPP synthase (FPPS). Aedes aegypti cDNAs encoding HMGS, PMK and FPPS were expressed as fusion proteins in Escherichia coli DH5 $\alpha$ cells, and purified by affinity chromatography. HMGS catalyzes the condensation of acetoacetyl-CoA and acetyl-CoA to produce HMG-CoA. The reaction did not require any co-factor, although the activity of the recombinant enzyme was enhanced by adding $\mathrm{Mg}^{2+}$. Hymeglusin, a specific $\beta$-lactone inhibitor of the vertebrate HMGS, inhibited HMGS enzyme activity in crude extracts from thoraces and abdominal carcasses of A. aegypti. AaPMK, a member of the nucleoside monophosphate family, catalyzes the cation-dependent reversible reaction of phosphomevalonate and ATP to form diphosphate mevalonate and ADP. Kinetics for both forward and backward reactions were determined, and we observed that the activity of AaPMK was not inhibited by any of the downstream metabolites. FPPS catalyzes the synthesis of different chain length isoprenyl diphosphates depending upon availability of metal cofactors. AaFPPS yielded $84 \% \mathrm{C}_{10^{-}}$-geranyl pyrophosphate (GPP) and $16 \% \mathrm{C}_{15^{-}}$ farnesyl pyrophosphate (FPP) in the presence of $\mathrm{Co}^{2+}$ as a cofactor, whereas it yielded $33 \%$ $\mathrm{C}_{10}$ GPP and $67 \% \mathrm{C}_{15}$ FPP in the presence of $\mathrm{Mg}^{2+}$. 


\subsection{Introduction}

\section{HMG-CoA synthase}

HMGS is the second enzyme of the JH pathway. It catalyzes the condensation of acetoacetyl-CoA and acetyl-CoA into HMG-CoA, releasing free CoA. Kinetic studies have established that this enzymatic step proceeds via a Bi-Bi Ping-Pong mechanism; where HMG-CoA is formed in three consecutive steps that include acetylation, condensation and hydrolysis (Fig. 28) (Miziorko and Lane, 1977; Pojer et al., 2006). Step 1 is the acetylation of acetyl-CoAS to an acetyl-S-enzyme intermediate. Step 2 involves the condensation of acetoacetyl-CoAS and acetyl-S-enzyme to form CoAS-HMG-Senzyme. Step 3 is the hydrolysis of CoAS-HMG-S-enzyme to produce HMG-CoA (Fig. 28) (Miziorko and Lane, 1977; Pojer et al., 2006). Acetylation is the rate limiting step in the synthesis of HMG-CoA, as the rate of acetylation of HMGS by acetyl-CoA is much slower than the hydrolysis of CoAS-HMG-S-enzyme (Miziorko and Lane, 1977).

Two isoforms of the enzyme have been identified in mammals; HMGS1 and HMGS2. HMGS1 is a cytosolic protein involved in isoprenoid/cholesterol biosynthesis, while HMGS2 is a mitochondrial protein involved in the biosynthesis of ketone bodies (Goldstein and Brown 1990; Dooley et al., 1998; Willamson et al., 1968; Dashti and Ontko, 1979). HMG-CoA reductase and HMGS1 are considered key regulatory enzymes in the biosynthesis of cholesterol (Goldstein and Brown 1990; Dooley et al., 1998). In insects, HMGS does not have any recognizable N-terminal targeting sequence to mitochondria, which suggests it is present in the cytosol (Buesa et al., 1994; Tittiger et al., 2000). Most insects have one HMGS gene, but Blattella germanica is an exception with 2 HMGS genes (HMGS1A and HMGS1B), showing 78\% similarity (Buesa et al., 
1994; Belles et al., 2003). HMGS1A is intronless, and expression studies and phylogenetic analysis suggest it represent a functional retrogene derived from HMGS1B by retrotransposition (Buesa et al., 1994; Cabano et al., 1997; Casals et al., 2001). In insects HMGS has been biochemically characterized only from the cockroach Blattella germanica (Buesa et al., 1994; Casals et al., 1996).

\section{Phosphomevalonate kinase}

Phosphomevalonate kinase (PMK) is the second ATP-dependent enzyme in the mevalonate pathway. It catalyzes the phosphorylation of phosphomevalonate (PM) to diphosphomevalonate (DPM) (Fig. 29). PMKs are related to various human diseases, such as Zellweger syndrome and rhizomelic chondrodysplasia punctata (Braverman et al., 1997; Wanders and Romeijn, 1998). However, in mammals, PMKs have not been as well characterized as are other enzymes involved in isoprenoid biosynthesis. Animal PMKs are encoded by genes that are non-orthologous to plant, fungal, and bacterial PMKs genes (Smit and Mushegian, 2000). Analysis of the crystal structure of Streptococcus pneumoniae PMK confirmed that this protein belongs to the GHMP kinase family (Romanowski et al., 2002; Andreassi et al., 2009). Mammalian PMKs have been purified and characterized from a variety of tissues (Hellig and Popjak 1961a, 1961b; Bazaes et al., 1980a, 1980b; Lee and O'Sullivan 1985; Chambliss et al., 1996; Herdendorf and Miziorko, 2006, 2007). Kinetic and biophysical studies suggest that the animal PMKs belong to the nucleoside monophosphate (NMP) family, which is also known as P-loop kinases family (Herdendorf and Miziorko, 2006, 2007).

In the P-loop kinases, the $\beta$ sheet is five stranded, with a highly conserved order of 23145 (Walker et al., 1982). There are two highly conserved motifs known as Walker 
A (GXXXXGK/T/S) and Walker B (ZZZZD, where $Z$ is any hydrophobic residue) (Cheek et al., 2002). The mechanism of reaction involves the Walker A motif forming a phosphate-binding loop (P-loop) that is located at the end of the first $\beta$-strand, and includes the first half-turn of the following $\alpha$-helix. The conserved lysine residue of the Walker A motif binds to and orients oxygen atoms of the $\beta$ and $\gamma$-phosphate groups of ATP (Cheek et al., 2002). The essential magnesium cation is coordinated directly by the hydroxyl group of the conserved threonine/serine of the Walker A motif, and indirectly by the conserved aspartate residue of the Walker B motif (Cheek et al., 2002).

\section{FPP synthase}

FPPS are prenyltransferases, also known as isoprenyl diphosphate synthases (IDS). Prenyltransferases catalyze the consecutive condensation of isopentenyl diphosphate (IPP) with allylic prenyldiphosphates, to yield products with chain lengths varying from $\mathrm{C}_{10}$ up to many $\mathrm{C}$. Based on the geometry of newly formed double bond ( $\mathrm{E}$ or Z), and the size of the isoprenoid chain in the final product, prenyltransferases can be classified as short chain and long chain prenyltransferases. GPP synthase (GPPS) produces GPP $\left(\mathrm{C}_{10}\right)$, FPPS produces $\mathrm{FPP}\left(\mathrm{C}_{15}\right)$ and geranyl-geranyl pyrophosphate synthase (GPPS) produces GGPP $\left(\mathrm{C}_{20}\right)$. They are classified as short chain Eprenyltransferases (Ogura and Koyama, 1998). The enzyme IDS, which produce compounds involved in respiratory quinone biosynthesis, with chain length varying from $\mathrm{C}_{30}$ to $\mathrm{C}_{50}$, are considered as long chain E-prenyltransferases (Okada et al., 1996). The Zpolyprenyl diphosphate synthases are involved in the synthesis of long chain dolichols and several other very long chain isoprenoids (Sato et al., 1999). These enzymes could be encoded by different genes, but they share the mechanism of reaction and well-defined 
highly conserved motifs characteristic of proteins evolved from a common ancestor (Chen et al., 1994; Fujihashi et al., 2001).

The crystal structure of FPPS has not been determined in insects, but X-ray structures have been resolved for a several organisms, including avian FPPS (Tarshis et al., 1994, 1996), Staphylococcus aureus FPPS, Escherichia coli FPPS (Hosfield et al., 2004), Trypanosoma cruzi FPPS (Gabelli et al., 2006), and human FPPS (Rondeau et al., 2006). These studies described the enzymes as homodimers, with each subunit folded in a single domain, whose central feature is a core composed of $10 \alpha$ - helix surrounding a large deep cleft which is identified as the substrate binding pocket.

The reaction mechanism for FPPS is postulated as a dissociative electrophilic alkylation, which is divided into three steps as shown in figure 30. In step 1, the C1oxygen bond in DMAPP or GPP ruptures to generate a resonance stabilized allylic cation. In step 2, this allylic cation alkylates the double bond in IPP to produce a tertiary carbocation. During step 3, hydrogen is subsequently eliminated from C2 of the IPP unit to produce a new allylic diphosphate which is one isoprene unit longer than the substrate (Fig. 30) (Poulter and Rilling, 1978; Poulter, 2006). The reaction depends upon the metal cofactor usually $\mathrm{Mg}^{2+}, \mathrm{Mn}^{2+}$ or $\mathrm{Co}^{2+}$ (Aaron and Christianson, 2010). In the beetle Phaedon cochleariae, FPPS shows an unusual product regulation mechanism; it alters the chain length of its products depending on the cofactor present. The FPPS yields $\mathrm{C}_{10}$-GPP in the presence of $\mathrm{Co}^{2+}$ or $\mathrm{Mn}^{2+}$, whereas it produces the longer $\mathrm{C}_{15}$-FPP in the presence of $\mathrm{Mg}^{2+}$ (Frick et al., 2013). 


\subsection{Methods}

\subsubsection{Insects}

A. aegypti of the Rockefeller strain were reared at $28{ }^{\circ} \mathrm{C}$ and $80 \%$ relative humidity under a photoperiod of $16 \mathrm{~h}$ light: $8 \mathrm{~h}$ dark. A cotton pad soaked in $3 \%$ or $20 \%$ sucrose solution was provided to adults.

\subsubsection{Tertiary structure of proteins}

The three dimensional model structures for AaPMK and AaFPPS were predicted using the crystal structures of human PMK (Protein Data Bank ID code 3ch4.1) and avaian FPPS (Protein Data Bank ID code 1ubx.1) respectively as templates. The identity of the human PMK and AaPMK was $49.08 \%$ and that of avian FPPS and AaFPPS was $47.35 \%$. The models were performed using the protein structure homology modeling server Swiss v.8.05 (Schwede et al., 2003; Arnold et al., 2006)

\subsubsection{Expression of recombinant proteins}

The cDNAs of AaHMGS, AaPMK and AaFPPS were expressed in E. coli cells as described by Nyati et al., 2013. Recombinant His-tagged proteins were purified using HiTrap chelating columns and PD-10 desalting columns (Amersham Pharmacia, Piscataway, NJ). Glycerol was added to the enzyme solution (final concentration 50\%), and samples were stored at $-20{ }^{\circ} \mathrm{C}$ until used. Protein concentrations were determined using the bicinchoninic acid protein assay reagent (BCA) (Pierce, Rockford, IL). Bovine serum albumin was used as a standard. 


\subsubsection{Enzymatic assays}

\subsubsection{PMK assays}

The catalytic activity of the AaPMK was measured using a modified spectrophotometric assay that couples ADP formation to pyruvate synthesis and reduction to lactate as described in chapter 4.

\subsubsection{FPPS assays}

AaFPPS assays were carried out in a final volume of $100 \mu \mathrm{L}$ containing $100 \mathrm{mM}$ Tris$\mathrm{HCl}(\mathrm{pH} 7.5), 100 \mu \mathrm{M}$ IPP, $100 \mu \mathrm{M}$ DMAPP, and $5 \mathrm{mM} \mathrm{Mg}^{2+}$ or $1 \mathrm{mM} \mathrm{Co}^{2+}$. Reactions were started with the addition of $4 \mu \mathrm{g}$ recombinant enzyme. Formation of FPP and GPP were monitored by transforming them into FOL and geraniol using $50 \mu \mathrm{L} 3 \mathrm{~N}$ HCL and quantifying them by reverse phase-HPLC as shown in chapter 3.

\subsubsection{HMGS assays}

The dependence of enzyme activity on the cofactor concentration was measured by monitoring the disappearance of acetoacetyl-CoA at $300 \mathrm{~nm}\left(\varepsilon_{\mathrm{mM}}=3.6\right)$ in $100 \mathrm{mM}$ Tris$\mathrm{HCl}$ at $30^{\circ} \mathrm{C}$ containing $200 \mu \mathrm{M}$ acetyl-CoA, $15 \mu \mathrm{M}$ acetoacetyl-CoA and $0,1,2,5$, or 10 $\mathrm{mM} \mathrm{MgCl}_{2}$. The $\mathrm{pH}$ dependency of AaHMGS activity was measured by the same assay by increasing the $\mathrm{pH}$ in 0.5 -unit increments from 7 to 9 using the acetoacetyl-CoA extinction coefficients $(300 \mathrm{~nm})$ appropriate for each $\mathrm{pH}$ value. To determine the $\mathrm{Km}$ of acetyl-CoA, $2 \mu \mathrm{g}$ of AaHMGS was incubated in $100 \mathrm{mM}$ Tris- $\mathrm{HCl}(\mathrm{pH} 8)$ at $30^{\circ} \mathrm{C}$ with $15 \mu \mathrm{M}$ acetoacetyl-CoA and acetyl-CoA concentrations ranging from 4 to $400 \mu \mathrm{M}$, and activity was determined by monitoring the disappearance of acetoacetyl-CoA at $300 \mathrm{~nm}$. The $\mathrm{Km}$ of acetoacetyl-CoA was determined using fixed concentration of acetyl-CoA $(250 \mu \mathrm{M})$ and acetoacetyl-CoA concentrations ranging from 0.25 to $100 \mu \mathrm{M}$. 


\subsection{Results and discussion}

\subsubsection{Structural analysis of AaPMK through molecular modelling}

AaPMK is a 187 amino acid protein comprised of three domains; the core region (residues 1-41, 100-107, 120-130 and 164-187), the LID region (132-164) and the substrate binding region (42-99, 108-118) (Fig. 32). It has in the center five-stranded parallel $\beta$-sheet, with a strand order of 23145, and eight $\alpha$-helices (Fig. 32). The structure of AaPMK discloses the typical NMP kinase fold (Fig. 32), similar to the structure of the enzyme from Homo sapiens (HsPMK) (Chang et al., 2008). During the PMK catalysis, the P-loop or Walker A motif is involved in binding with the triphosphate, the substrate binding region binds with $\mathrm{PM}$, and the LID region has an opening and closing motion, to permit the binding and release of substrates (Chang et al., 2008; Olson et al., 2009).

\subsubsection{Characterization of the activity of AaPMK}

A PMK has been kinetically characterized for the first time in insects. The proper expression of the functional AaPMK protein was verified by SDS-PAGE and western blot (Fig. 31). AaPMK activity was investigated at different pHs. The optimum $\mathrm{pH}$ for AaPMK was 7.5, which decreases in activity at $\mathrm{pHs}$ lower than 6.5 and higher than 8 (Fig. 33A). AaPMK shows a cation dependence on $\mathrm{Mg}^{2+}$, with a $2 \mathrm{mM}$ concentration revealing the maximal activity (Fig. 33B). Kinetic constants were determined by nonlinear regression analysis using the GraphPad Prism Software. The $K_{\mathrm{m}}$ values for ATP and MA were $104 \pm 11$ and $190 \pm 16$ respectively. The $K_{\mathrm{m}}$ values for PM and ADP were respectively $180 \pm 39$ and $107 \pm 29$. The $V_{\max }$ was $7.9 \pm 0.8 \mu \mathrm{mol} / \mathrm{min} / \mathrm{mg}$ enzyme for the forward reaction, and $3.1 \pm 1.2 \mu \mathrm{mol} / \mathrm{min} / \mathrm{mg}$ enzyme for the backward reaction. The kinetics constants for the PMKs from the bacteria Enterococcus faecalis (EfPMK) (Doun 
et al., 2005) and Streptococcus pneumonia (SpPMK) (Pilloff et al., 2003), the yeast Saccharomyces cerevisiae (SCPMK) (Garcia and Keasling, 2014) and the mammals Sus domesticus (SdPMK) (Eyzaguirre et al., 2006) and Homo sapiens (HsPMK) (Herdendorf and Miziorko, 2006) are compared with the AaPMK kinetic constants in table 5.

Addition of GPP and FPP did not affect AaPMK activity at concentrations up to $200 \mu \mathrm{M}$, hence feedback inhibition mechanisms can be ruled out for AaPMK.

\subsubsection{Molecular model of AaFPPS}

Based upon the available crystal structure of an avian, Gallus gallus FPPS (GgFPPS) (Tarshis et al., 1994), the 3-D structure of AaFPPS was modeled (Fig. 34). The homology model of AaFPPS shows that protein folded as a single domain, composed of all antiparallel $\alpha$-helixes and no $\beta$-sheets (Fig. 34). Other enzymes, which also utilize isoprenyl diphosphate as their substrate, such as squalene cyclase, 5-epi-articolochene synthase, pentalenene synthase and protein farnesyl transferase, showed similar core structures formed only by $\alpha$-helices (Wendt et al., 1997; Starks et al., 1997; Lesburg et al., 1997; Long et al., 1998). Hence these structures of proteins have been given the name 'terpenoid synthase fold' (Wang and Ohnuma, 2000).

Amino acid sequence alignments of FPPS from four insect species including Aedes aegypti (AaFPPS), Culex quinquefasciatus (CqFPPS), Anopheles gambiae (AmFPPS), and Drosophila melanogaster (DmFPPS) revealed the sequences of the five well conserved motifs typical of E-prenyltransferases (Fig. 35). The avian, Gallus gallus FPPS (GgFPP) was also used for comparison, since its crystal structure is known (Tarshis et al., 1994) and has close similarity with the sequence of AaFPPS (44\%). The AaFPPS contains five conserved motifs found in other insects, as well as in the avian 
FPPS (Fig. 8). The sequence of AaFPPS revealed two prominent aspartate rich motifs, DDXXD, known as FARM (first aspartate rich motif) and SARM (second aspartate rich motif), located in the II and V motif respectively (Fig. 35). The presence of FARM and SARM motifs represents a typical feature of E- prenyltransferases, and they have also been reported in the sequences of other enzymes that use isoprenyl diphosphate as substrate, such as monoterpene cyclases, sesquiterpene cyclases, and diterpene cyclase. The FARM motif and the first two aspartate residues in the SARM are involved in the enzyme catalytic mechanism via a metal cofactor; while the last aspartate residue is involved only in binding but not in the catalytic efficiency of the enzyme (Marrero et al., 1992; Joly et al., 1993; Song et al., 1994; Koyama et al., 1996). In addition motifs I and IV are also involved in binding with IPP, as experiments have demonstrated a very high fold increase in $K_{\mathrm{m} \text { IPP }}$ of enzyme when the conserved lysine is mutated into an aliphatic amino acid in Bacillus stearothermophilus FPP synthase (Koyama et al., 1996).

\subsubsection{Partial characterization of AaFPPS activity}

The expression of a functional AaFPPS protein was verified by SDS-PAGE and western blot (Fig. 36). Optimum $\mathrm{pH}$ values for the FPPS previously characterized from other insects such as cotton boll weevil, Anthonomus grandis (Taban et al., 2009) and horseradish leaf beetles, Phaedon cochleariae (Frick et al., 2011) was 7.5 in 50 mM Tris$\mathrm{HCl}$ buffer; hence the activity of AaFPPS was also measured under similar conditions of buffer and $\mathrm{pH}$. AaFPPS yielded $84 \% \mathrm{C}_{10^{-}}$geranyl pyrophosphate (GPP) and $16 \% \mathrm{C}_{15^{-}}$ farnesyl pyrophosphate (FPP) in the presence of $\mathrm{Co}^{2+}$ as a cofactor; whereas it yielded $33 \% \mathrm{C}_{10}$ GPP and $67 \% \mathrm{C}_{15} \mathrm{FPP}$ in the presence of $\mathrm{Mg}^{2+}$ (Fig. 36). This unusual product regulation mechanism by metal cofactor was also observed in the beetle Phaedon 
cochleariae FPPS (Frick et al., 2013). The physiological significance of the product regulation of FPPS by cofactors is unknown. In A. aegypti $24 \mathrm{~h}$ before adult eclosion, the pool of GPP is very high $(50,000 \mathrm{fmol} / \mathrm{CA})$ and the FPP pool is low $(60 \mathrm{fmol} / \mathrm{CA})$ (Rivera-Perez et al., 2014). After the adult eclosion the size of the GPP pool decreases, and GPP becomes undetectable; while the FPP pool fluctuated from a low of 10 to a high of $100 \mathrm{fmol} / \mathrm{CA}$, depending upon the physiological state of the CA (Rivera-Perez et al., 2014).

\subsubsection{Characterization of the AaHMGS activity}

The expression of the functional AaHMGS protein was verified by SDS-PAGE and western blot (Fig. 31). The activity of AaHMGS was measured using both the DNTBCoA assay and the disappearance of acetoacetyl-CoA at $300 \mathrm{~nm}$. The AaHMGS activity was investigated at different pHs. The optimum pH for AaHMGS was 8-8.5 (Fig. 37). This value is comparable to the $\mathrm{pH}$ 9.4, reported for the avian liver cytoplasmic HMGS, (Clinkenbeard et al., 1975), the plant Brassica juncea HMGS (pH 8.5) (Nagegowda et al., 2004), the bacterial E. faecalis HMGS (pH 9.8) (Sutherlin et al., 2002) and the archaeal Haloferax volcanii HMGS (pH, 8.5) (VanNice et al., 2013). The HMGS does not require any co-factor; however the activities of the animal recombinant enzymes were enhanced by adding $\mathrm{Mg}^{2+}$. The AaHMGS was $180 \%$ activated in the presence of $5 \mathrm{mM}$ $\mathrm{Mg}^{2+}$, similar to that avian liver cytoplasmic HMGS (Clinkenbeard et al., 1975) and the insect, Blattella germanica (Cabona et al., 1996). On the contrary, the activity of the plant, Brassica juncea HMGS did not show any increase in the presence of metal cofactor (Nagegowda et al., 2004). 
AaHMGS exhibited a $V_{\max }$ of $3.3 \mu \mathrm{mol} / \mathrm{min} / \mathrm{mg}$ enzyme, and the $K_{\mathrm{m}}$ for acetylCoA and acetoacetyl-CoA were $98 \mu \mathrm{M}$ and $14 \mu \mathrm{M}$ respectively. These values were in the range of $V_{\max }$ and the $K_{\mathrm{m}}$ values reported for HMGSs of different organisms. Hymeglusin is a fungal metabolite that exhibits high specificity for inhibition of the HMGS activity (Tomoda et al., 1988). It inhibits the activity of the recombinant enzyme as well as HMGS activity in crude extracts from thorax and abdominal carcass of A. aegypti (Fig. $38)$.

\subsubsection{HMGS expression and activity in the abdominal carcass of $0 \%$ Vs $20 \%$ sugar fed mosquitoes}

Expression of AaHMGS is found in almost all female mosquito tissues (Nouzova et al., 2011). It has been shown in various organisms that expression of HMGS transcripts can be varied with source of nutrients. In the mycelia of fungi Ganoderma lucidum, HMGS expression profile analysis revealed that signaling molecules such as salicylic acid, abscisic acid and methyl jasmonate up regulated GlHMGS transcript levels (Ren et al., 2013). In addition carbon source has significant effects on GlHMGS transcript levels (Ren et al., 2013). Similarly, expression of AaHMGS is significantly reduced with silencing of TOR (Meritxell-Perez et al., 2013). In contrast we found that AaHMGS transcripts do not change with the nutritional condition of the insects (Fig. 39), but its activity gets significantly reduced when mosquitoes are starved for three days (Fig. 40).

\subsection{Conclusions}

We have started the characterization of Aedes aegypti HMGS, PMK and FPPS, three additional enzymes in the $\mathrm{JH}$ pathway. These studies will be the foundation for 
further analyses. They need to be completed in the future, but they are already revealing some interesting features that might be relevant for a better understanding of JH synthesis in mosquitoes. 


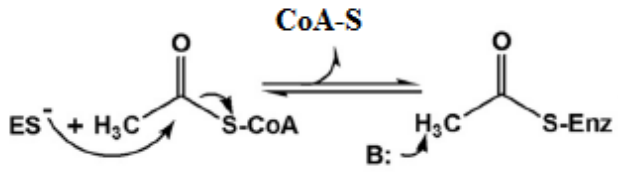

Acetyl-SCoA

Acetyl-S-Enzyme
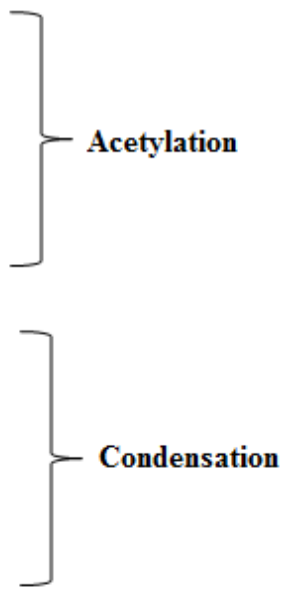

Enzyme-S-HMG-SCoA

Acetoacetyl-SCoA

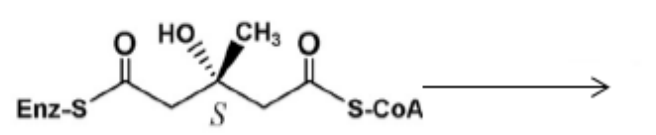<smiles>CC(C)(O)CC(=O)SC(=O)O</smiles>

HMG-SCoA

Figure 28: Reaction catalyzed by HMG-CoA synthase. HMGS catalyzes the formation of HMG-CoA in three steps: acetylation, condensation, and hydrolysis (Miziorko and Lane, 1977; Pojer et al., 2006). Step 1 is the acetylation of acetyl-CoAS to acetyl-S-enzyme intermediate, step 2 involves the condensation of acetoacetyl-CoAS and acetyl-S-enzyme to form CoAS-HMG-S-enzyme, and step 3 is the hydrolysis of CoAS-HMG-S-enzyme to produce $\mathrm{HMG}-\mathrm{SCoA}$. 


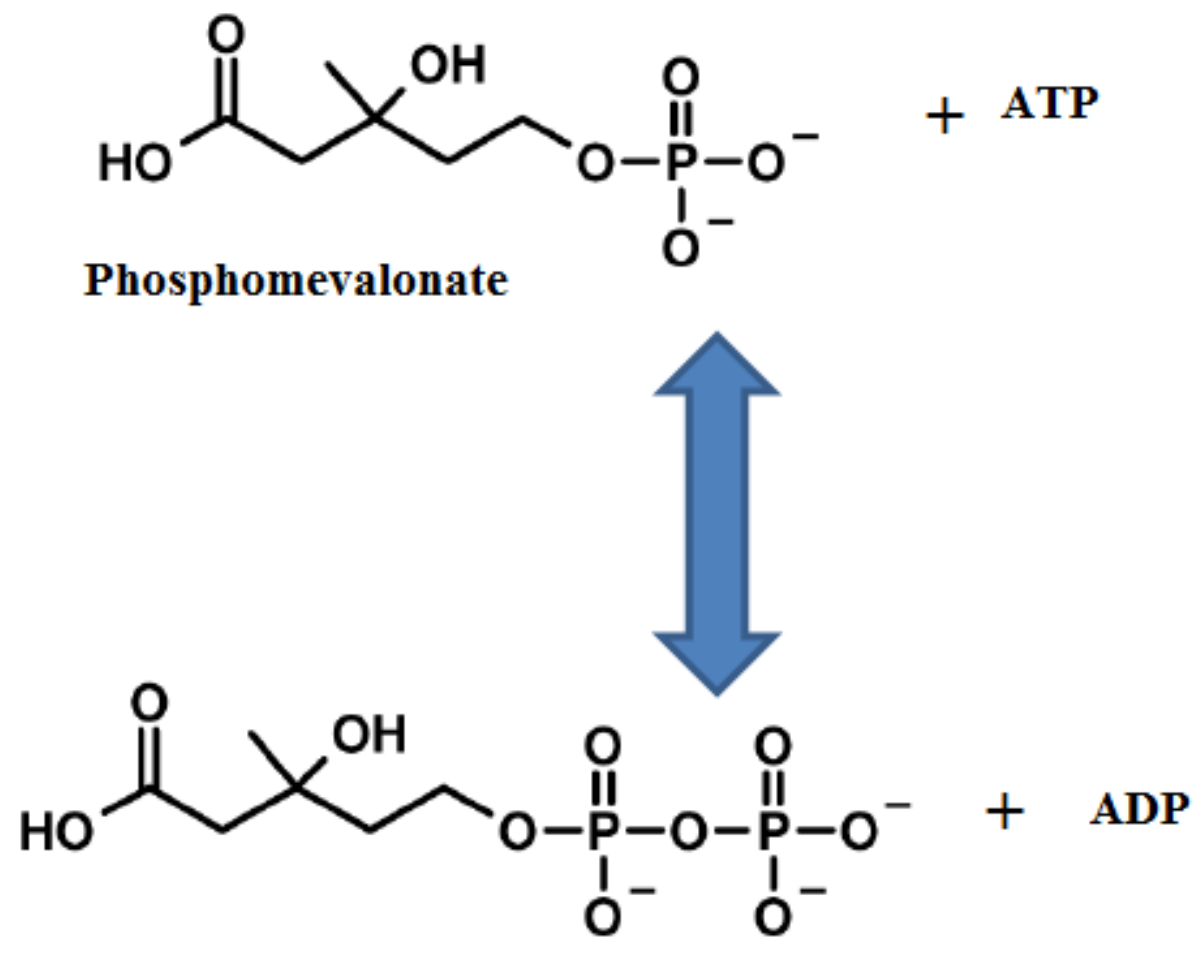

\section{Diphosphomevalonate}

Figure 29: Reaction catalyzed by PMK. AaPMK catalyzes the cation-dependent reversible reaction of phosphomevalonate and ATP to form diphosphate mevalonate and ADP. 

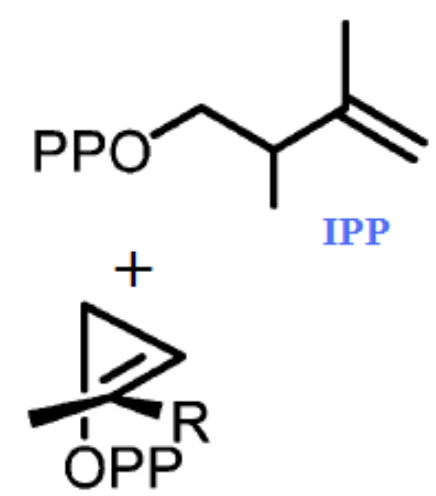

DMAPP or GPP

IPP
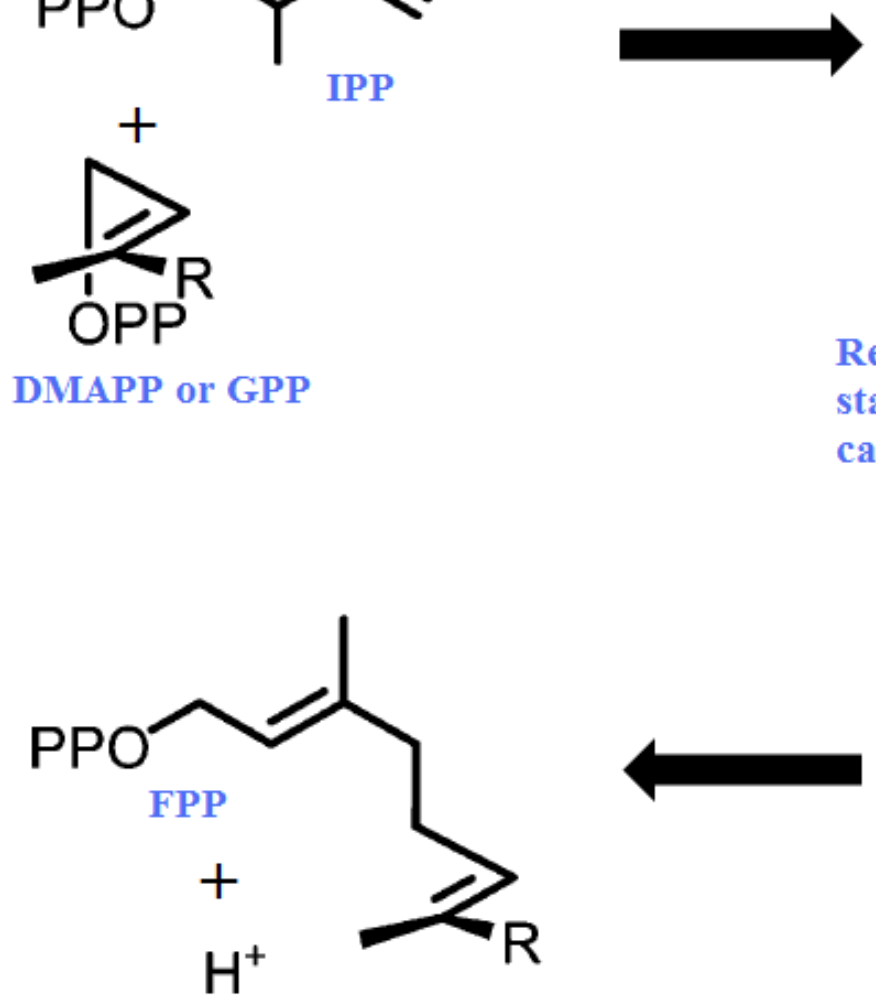
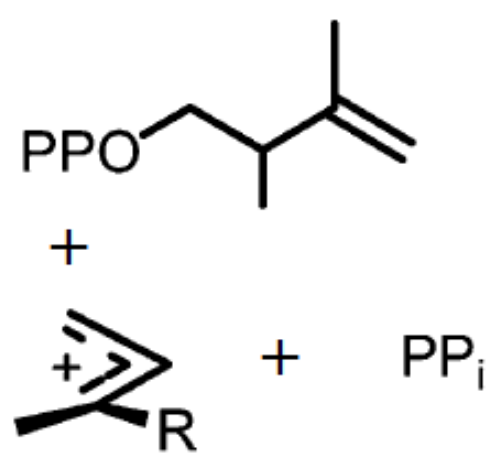

Resonance stabilized allylic cation

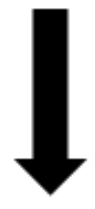

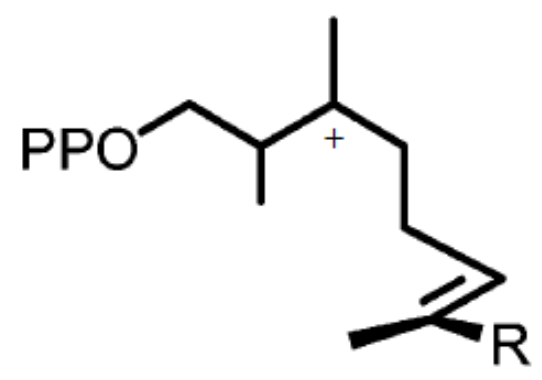

Tertiary carbocation

\section{$\mathrm{R}=\mathrm{CH}_{3}, \mathrm{C}_{6} \mathrm{H}_{11}$}

Figure 30: Reaction catalyzed by FPP synthase. FPPS catalyzes the dissociative electrophilic alkylation of IPP and DMAPP or GPP, which is divided into three steps. In step 1, the C1-oxygen bond in DMAPP or GPP ruptures to generate a resonance stabilized allylic cation. In step 2, this allylic cation alkylates the double bond in IPP to produce a tertiary carbocation. During step 3, hydrogen is subsequently eliminated from C2 of the IPP unit to produce a new allylic diphosphate which is one isoprene unit longer than the substrate (Poulter and Rilling, 1978; Poulter, 2006). 


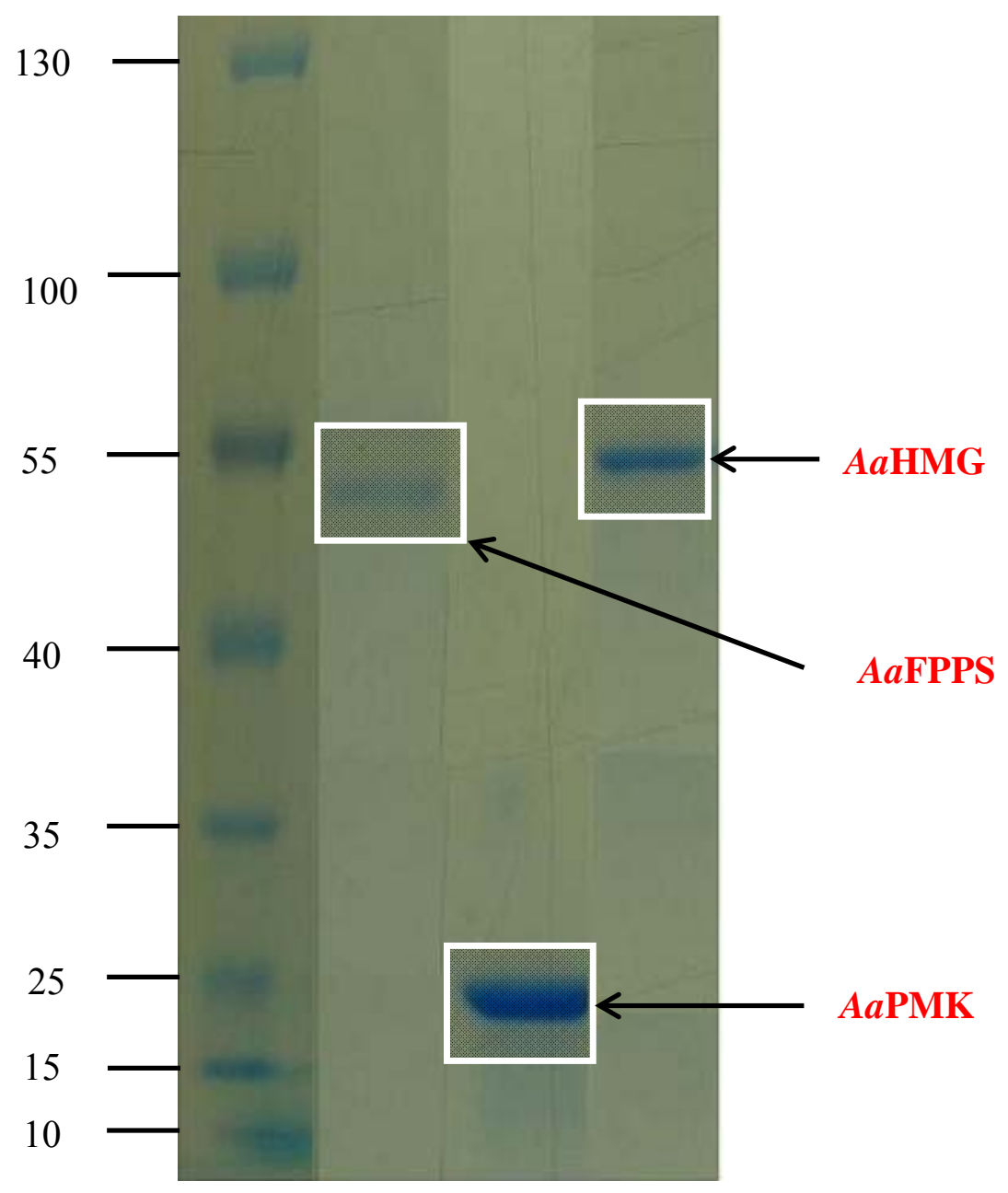

Figure 31: SDS-PAGE analysis of the recombinant mosquito AaHMGS, AaPMK and AaFPPS. Lanes are: 1, molecular standard; 2, AaHMGS; 3, AaPMK; 4, AaFPPS. The molecular weights of the protein standard are depicted on the $\mathrm{Y}$ axis. 


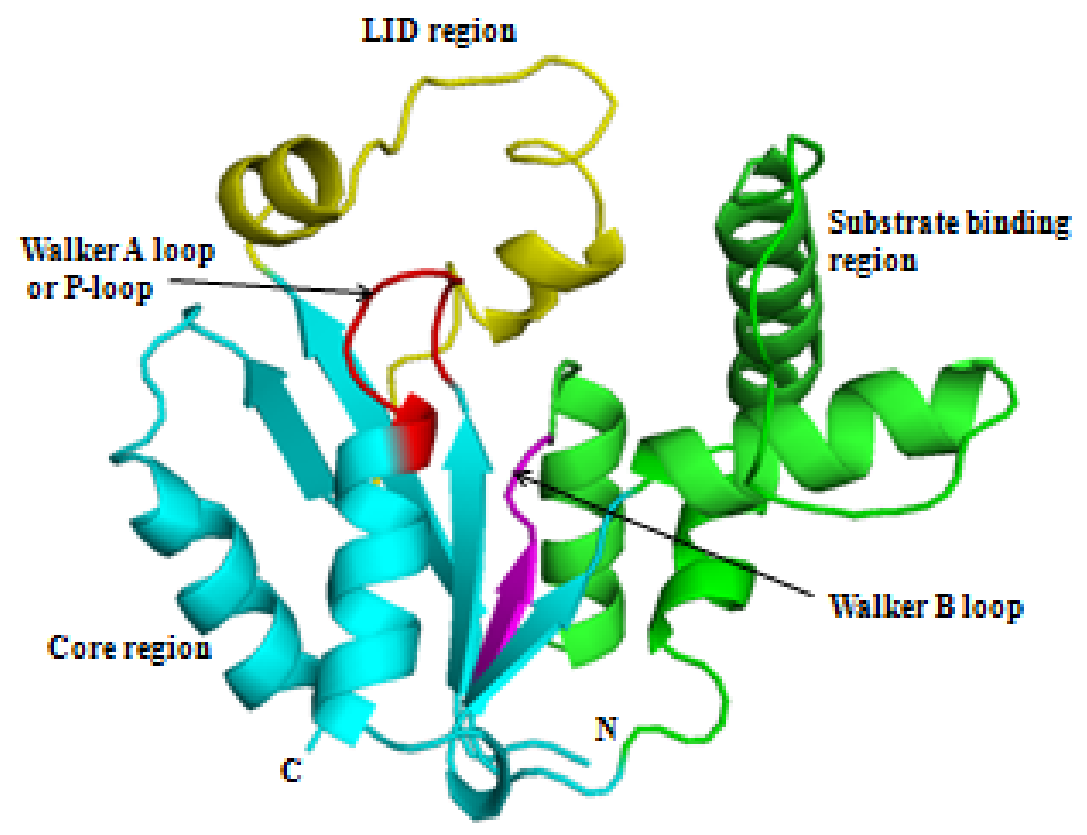

Figure 32: Homology model of the overall fold of AaPMK. The core region is shown in cyan, substrate binding region is shown in green, LID region is shown in yellow, Walker A loop motif or P-loop is shown in red, and Walker B motif is shown in pink. 

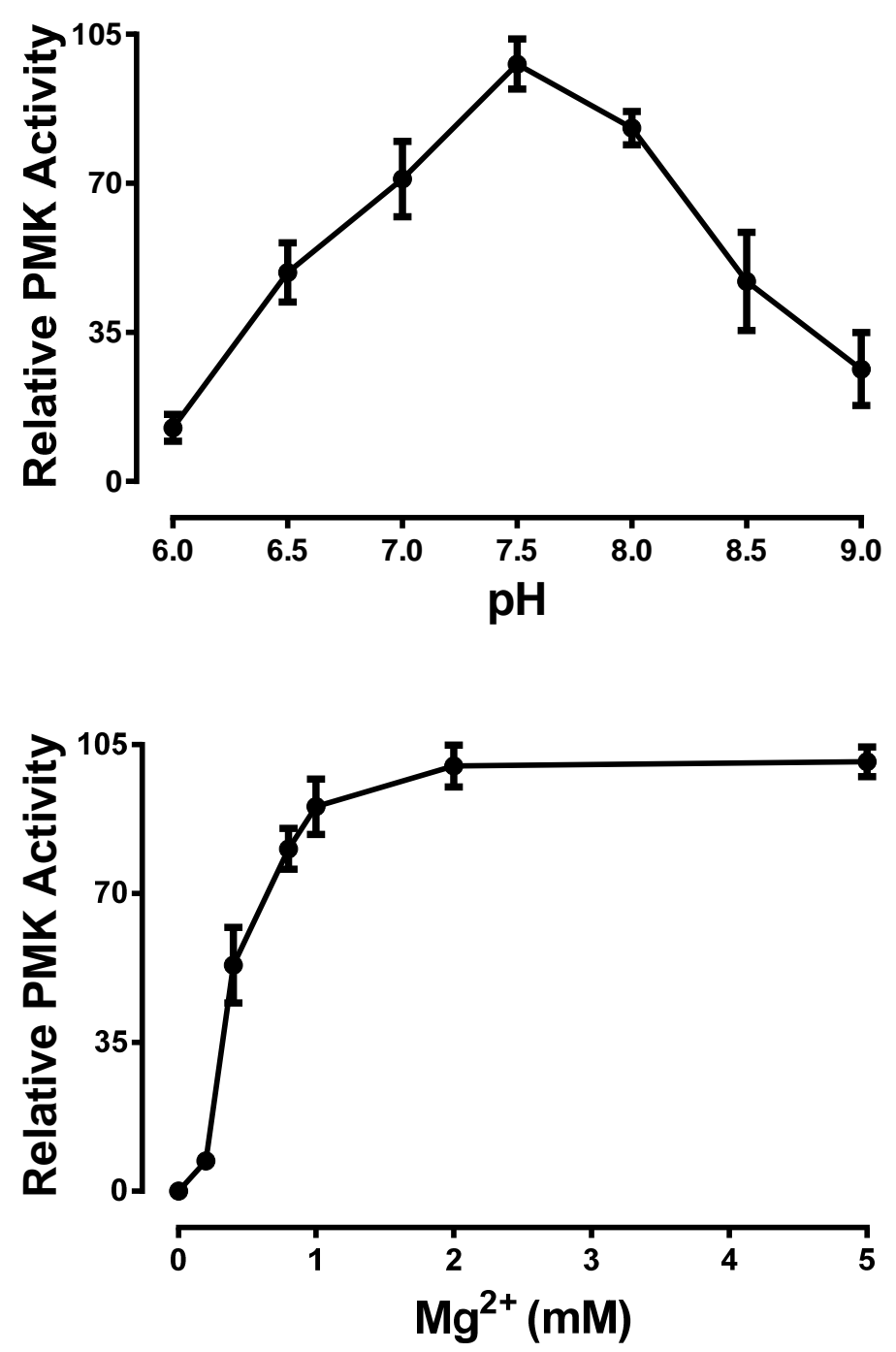

Figure 33: Characterization of AaPMK activity. The kinase activity was measured by an enzyme coupled spectrophotometric assay; ATP and PM were held constant, and data were normalized to the maximum observed reaction velocities. To ensure that PMK was the rate-limiting enzyme, when necessary the results were verified with the following conditions: doubling the amount of PMK added doubled the observed rate, doubling the amount of PK and LDH did not affect the observed rate, and doubling the PEP concentration did not affect the observed rate. (A) $\mathrm{pH}$ curve. Two different buffers were used: MES at $\mathrm{pH} 6$ to 7 and Tris- $\mathrm{HCl}$ at $\mathrm{pH} 7$ to 9. (B) Effect of metal cofactors $\mathrm{Mg}^{2+}$ was assayed for the AaPMK activity. Each value represents the means \pm S.E. of three replicate assays. Relative activity is defined as a percentage of the highest value recorded. 


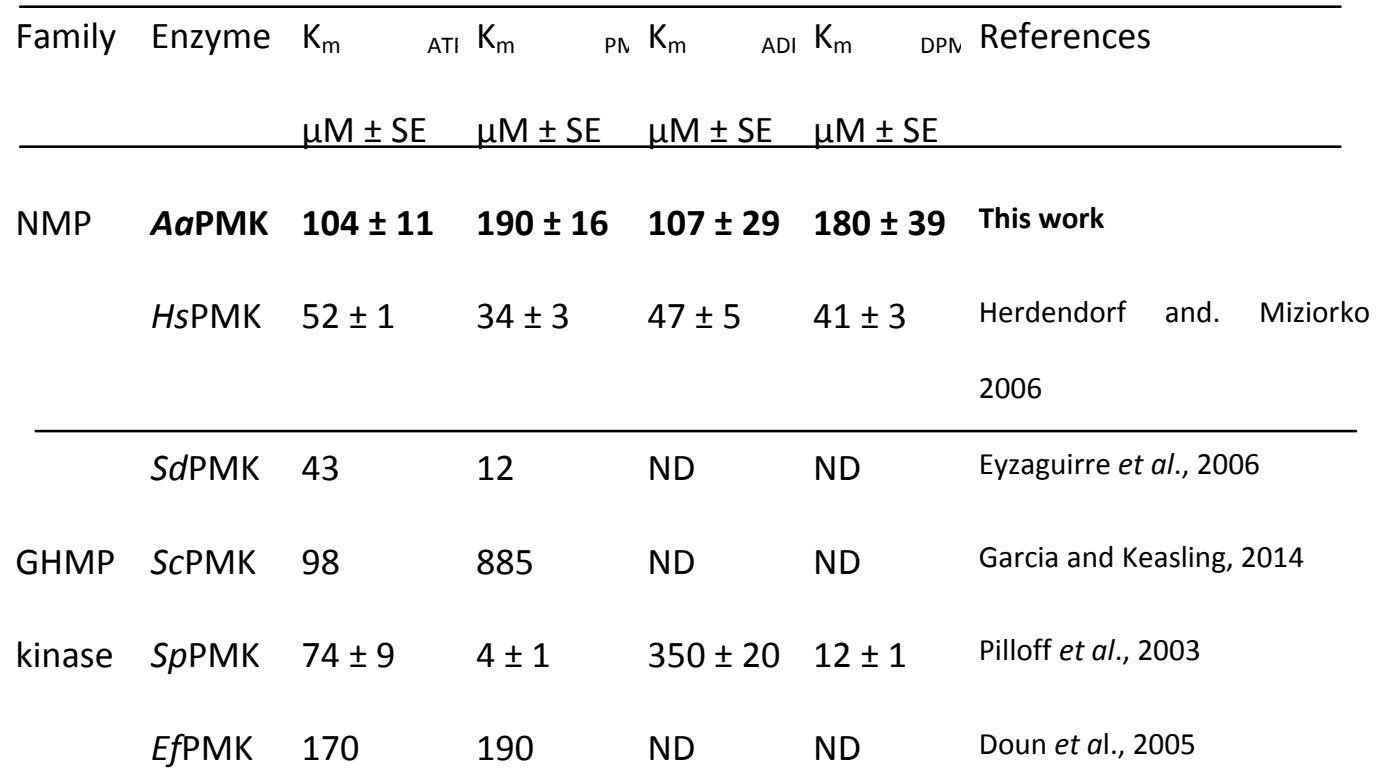

Table 5: Kinetics of PMK. AaPMK kinetics were compared with the kinetics of HsPMK (Homo sapiens PMK), SdPMK (Sus domesticus PMK), ScPMK (Saccharomyces cerevisiae PMK), SpPMK (Streptococcus pneumoniae PMK), EfPMK (Enterococcus faecalis $\mathrm{PMK}$ ). 


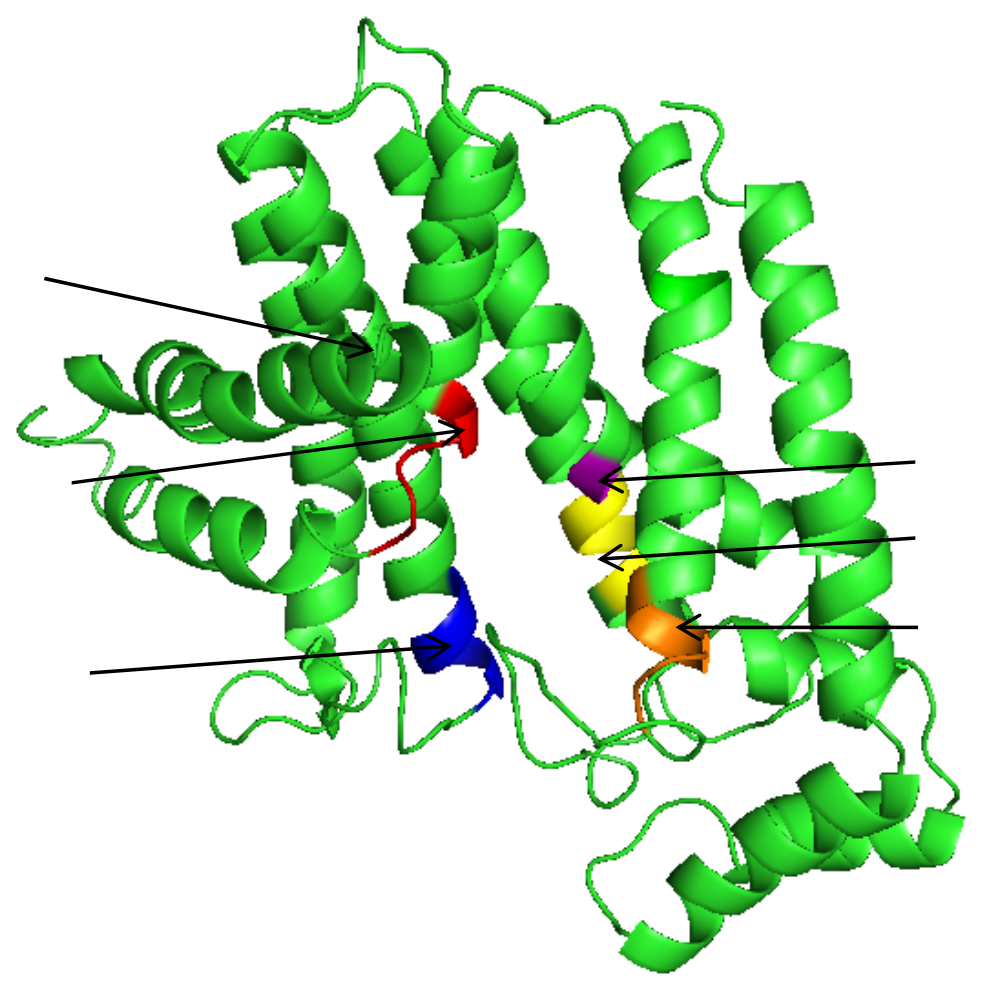

Figure 34: Homology model for the overall fold of AaFPPS. Three dimensional structure of AaFPPS. Motifs are indicated by colors; motif I (red), motif II (blue), motif III (yellow), motif IV (purple), motif V (Orange). The structure was constructed by PyMOL using the avian FPPS (PDB: 1ubx.1) as template. 
AaFPPS

CqFPPS

AgFPPS

DMFPPS

GgFPPS

AaFPPS

CqFPPS

AgFPPS

DMFPPS

GgFPPS

AaFPPS

CqFPPS

AgFPPS

DIFPPS

GgFPPS

AaFPPS

CqFPPS

AgFPPS

DMFPPS

G $g$ FPPS

AaFPPS

CqFPPS

AgFPPS

DMFPPS

GgFPPS

AaFPPS

CqFPPS

AgFPPS

DMFPPS

GgFPPS

AaFPPS

CqFPPS

AgFPPS

DIFPPS

GgFPPS

AaFPPS

CqFPPS

AgFPPS

DMFPPS

GgFPPS

- MLSTLIRAGS - - - - LQTMGRQFARSVETPVQMQHARFISKSSEVN - - - - - - - SDYMTM 47

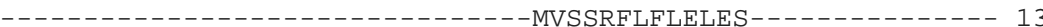
MSLFNLARFGVGVALEAAITRTAAPITATSGVVQLRRSISKSSEVNNSDFMTIRTENQNH 60 - -MFKLARMLLP - - - - - - - QQRILASP - - LRLQRLISTSDEVN - - - - - -AEPIIK 38

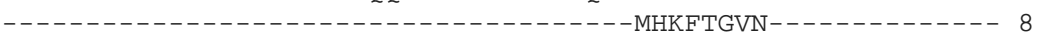

RENQTQKVSRDYNSKLQVRLKKVSRTLSTLNNSIPEAASQTIVTKSDAREFMAVFPDLVR 107 FLNRTDKII - - - - - - - - - - - TLSTLNCSVPEAATHTAVSKSESREFMAVFPDLVR 57 PHHHQKGSSRNCDSKQQIRLKKVSRTLSTLNSSVPEAATQTAVPKSESREFMAVFPDVVR 120 SMDTIGGLPTELVNEQ - -KLKKTSRTLSTLQNHSVPIAARVTVSKDESRDFMAVFPDLVR 96 - - - - AKFQQPALRNLSPVVVER-EREEFVGFFPQIVR 40 $\therefore:$ :

I

DLTEYCKKYDNT - LAPKWFVKALQYNVPQGKKNRGLAAVLAYRMLSKSEDLTPENIRRAH 166 DLTDYIKKYDEK - VAAKWFARALQYNVPQGKKNRGLAAVLAYRMLAKSHELTPENIRRAH 116 DLTAYASKYDKN - VATKWFVKALQYNVPQGKKNRGLACVLAYRMLARSEDLTPENIRRAQ 179 DITTVTKAYNCS - DAAKWFAQVLQYNVPRGKKNRGILTVLTYKNLVPTQDLTPENIKLAQ 155 DLTEDGIGHPEVGDAVARLKEVLQYNAPGGKCNRGLTVVAAYRELSGPGQKDAESLRCAL 100

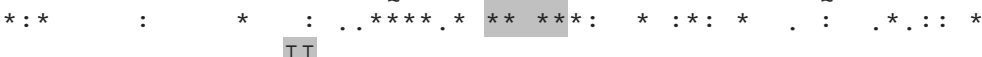

YLGWVIEMFQAVFLICDDAMDGSQTRRGQPCWYKLEDVKLSC INDAL YIDAAIFYVLKKQ 226 YLGWCIEMFQSVFLICDDVMDGSQTRRGQPCWYKVDDVKLTA VNDAL YLDAAIFHVLKKQ 176 YLGWAIEMLHSMFLIMDDVMDGSVTRRGQPCWHTLDDVKLSC VNDAI YIEAAIAHLVKIQ 239 YLGWCVEMLQSFFIISDDVMDNSTTRRGQPCWHKVENVGLTA INDAL YIENAMYAILKKH 215 AVGWCIELFQAASLVADDIMDQSLTRRGQLCWYKKEGVGLDF INDSF LLESSVYRVLKKY 160 $:{ }^{*}:{ }^{*}::::::^{*} * * * * * * * * *: .: *^{*} \ldots{ }^{*}, \ldots::::::^{*}$ III IV

FGDEPYYSKLVETFNEIKFITTIGQSLDLRSA - - RMDVTKYTMDLYKSIVCHKTAYYTF 283 FGDEPYYNKLVEMFNEIKFITTVGQSLDLQSA - - KLDVTQYTMDLYKSIVSHKTAYYTF 233 YGNEPYYPRLLELFNEMKFITTIGQSLDLRSA - - KLDVTDYSMDLYKSIVFHKTAYYTF 296 FSHLDCYVALMELFHEITYITTCGQSLDQLNS - - NRCVSEFTMENYKAIVENKTAYYSF 272 CRQRPYYVHLLELFLQTAYQTELGQMLDLITAPVSKVDLSHFSEERYKAIVKYKTAFYSF 220

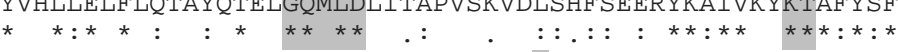

$\mathrm{V}$

YLPVALAMHMTGFTDPEVFRQTKTILLEIGLFYQTQDDFLDCFGDPAVTGKIGTDIEEGK 343 YLPVALAMHMTGFNDPEVFRQTKTILLEIGRFFQAQDDFLDCFGDPAVTGKIGTDIEEGK 293 YLPVAMAMHLTGYTDPEMFRQAKTILLEIGQFYQTQDDFFDCFGDPAVIGKVGTDIAEGK 356 YLPFALALHLAGYKDAEAFRQSKTILLEMGNFFQVQDDFLDCFGNPEVTGKIGTDIQDNK 332 YLPVAAAMYMVGIDSKEEHENAKAILLEMGEYFQIQDDYLDCFGDPALTGAVGTDIQDNK 280

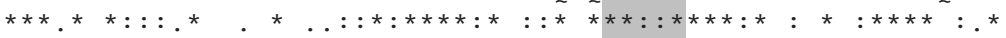

CTWLSVVAMQRASDEQKELMKQCYGSSDPEKVARVKKLYEELGLPTTYAIYEEESYNMIK 403 CTWLAVVCMQRASDEQKDIMKEFYGSSDPEKVARVKKLYEELGLPTTYAIYEEESYNIIK 353 CSWLAVVAMQRATEEQKEVMKACYGSTDPENIARVKKLYEQLGLPTTYSIYEEESYNMIK 416 CSWLAVVAMQRANVEQKQIMVDCYGKEEPAKVERVKELYKELGLPSTYAIFEEESYNMIK 392 CSWLVVQCLQRVTPEQRQLLEDNYGRKEPEKVAKVKELYEAVGMRAAFQQYEESSYRRLQ 340

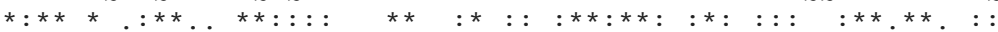

THIQQISRGLPHELFFKIMEKIYRRDC 430 THIQQISRGLPHELFFKIMEKIYRRDC 380 THIQQISRGLPHELFFKIMEKIYRREA 443 THIQQTSRGVPHQTFLQILNKIYQRDS 419 ELIEKHSNRLPKEIFLGLAQKIYKRQK 367 * : : *. : *: : *: : ${ }^{* * *}:{ }^{*}:$

Figure 35: Sequence alignment of the deduced amino acid sequence from insect FPPSs. The sequence of AaFPPS was aligned with insect FPPS from Culex quinquefasciatus (CqFPPS), Anopheles gambiae (AgFPPS), Drosophila melanogaster (DmFPPS), and the avian Gallus gallus (GgFPPS). Identical residues are indicated by an asterisk. The box indicates five highly conserved regions. Accession number AaFPPS (AAEL003497), AgFPPS (AGAP007104), CqFPPS (EDS25700.1), DmFPPS (NP_477380.1), GgFPPS (1UBV_A) 

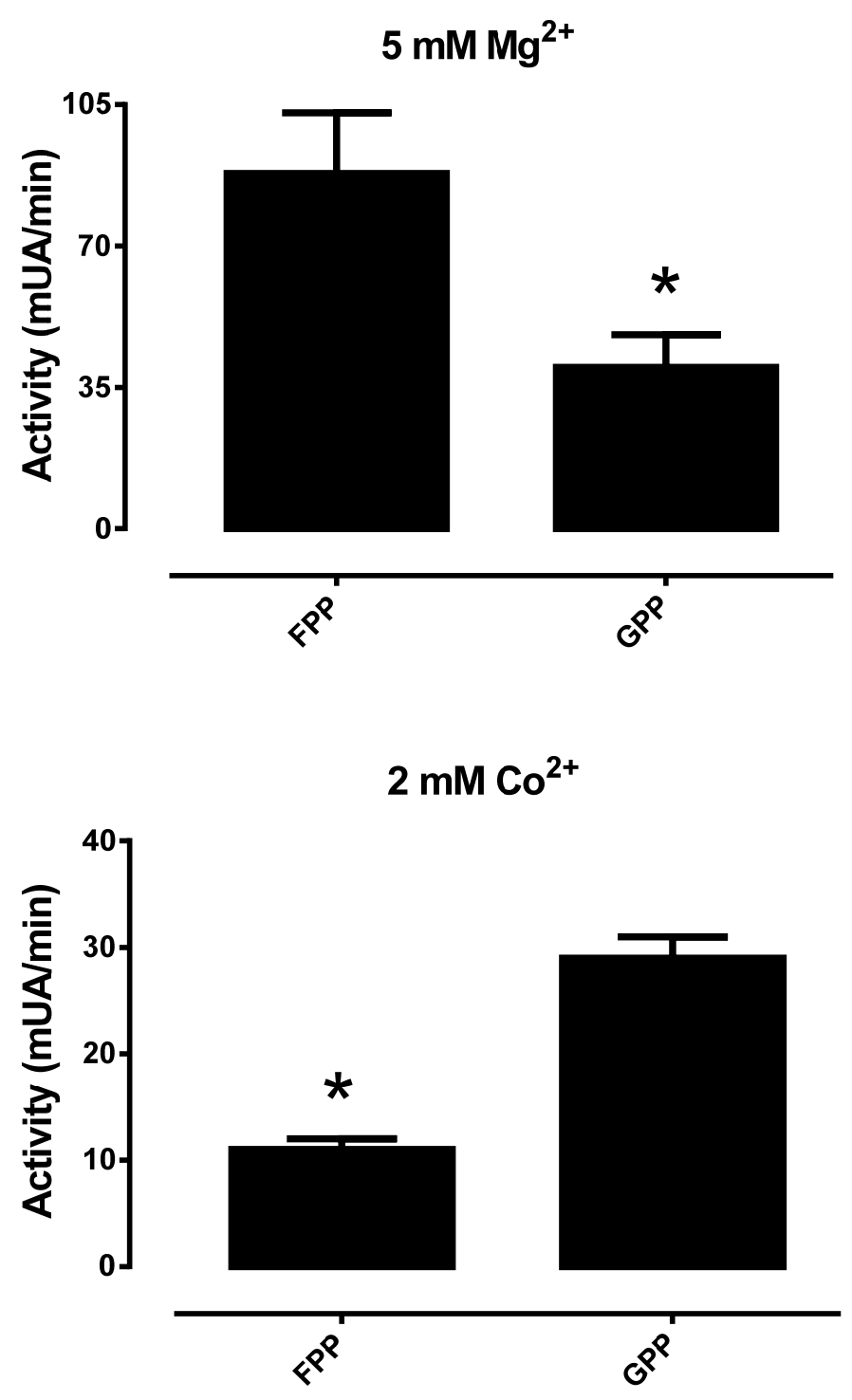

Figure 36: Effect of metal cofactors on enzyme activity and product formation of AaFPPS. $100 \mu \mathrm{M}$ IPP and $100 \mu \mathrm{M}$ DMAPP were incubated in presence of $5 \mathrm{mM} \mathrm{Mg}^{2+}$ (A) or $1 \mathrm{Mm} \mathrm{Co}^{2+}$ (B). Reactions were started with the addition of $4 \mu \mathrm{g}$ recombinant enzyme. Formation of FPP and GPP were measured as described in method section. 

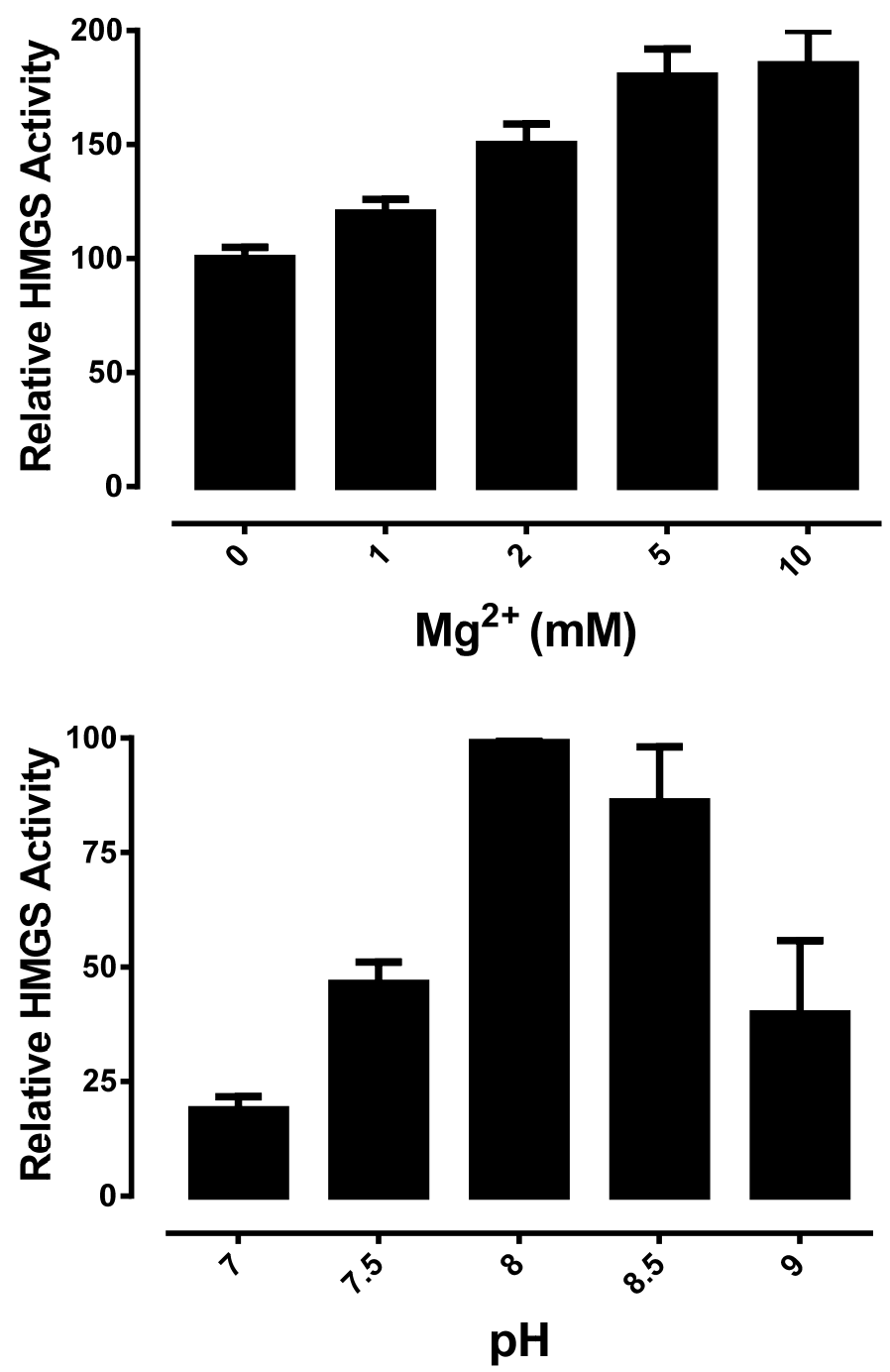

Figure 37: Characterization of AaHMGS activity A) Magnesium dose-dependent increases of activities. B) Optimum $\mathrm{pH}$ determinations. Each value represents the means \pm S.E.M. of three replicate assays. Relative activity is defined as a percentage of the highest value recorded. 


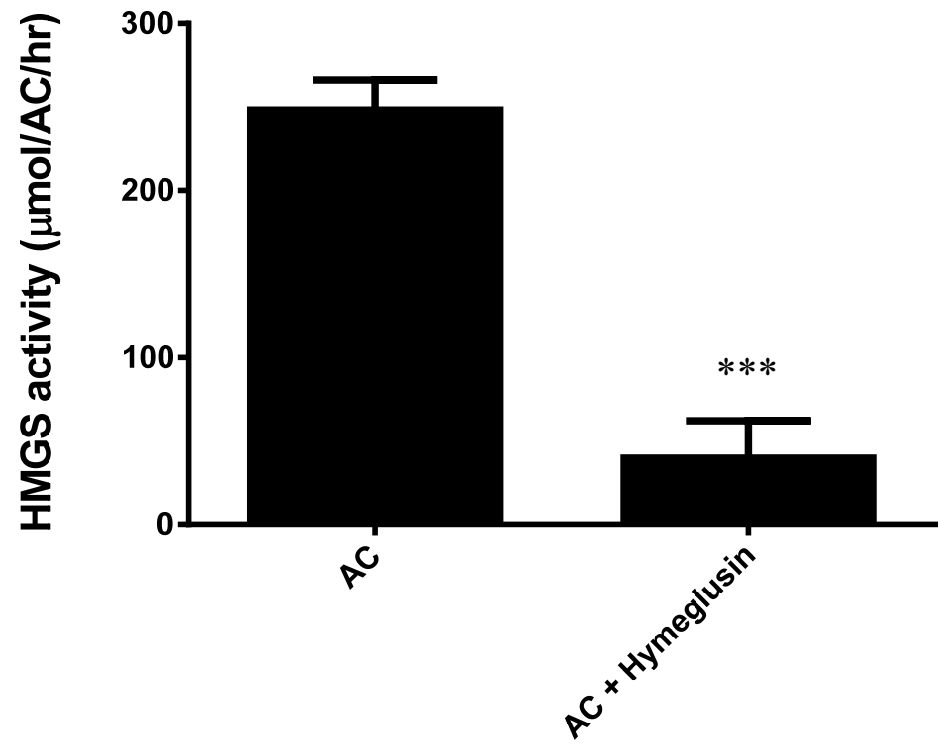

Figure 38: Inhibition of AaHMGS abdominal carcass activity by hymeglusin. The reaction was performed by the DNTB-CoA assay. $1 \mathrm{mg}$ of protein was added in $24 \mathrm{~h}$ $20 \%$ SF females after adult eclosion in the presence and absence of hymeglusin. Each point $( \pm$ SEM) represents the mean of 3 biological replicates of extracts from $5 \mathrm{AC}$ from female mosquitoes. Significant differences $(p<0.01)$ were determined with one way ANOVA followed by Tukey's test. 


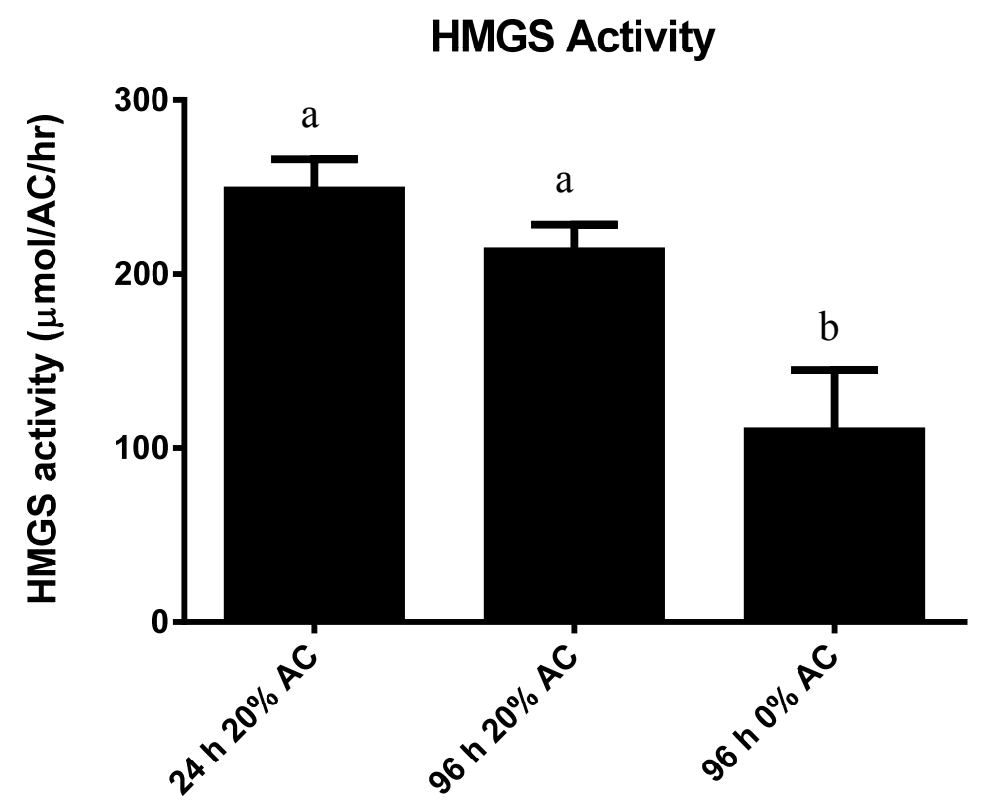

Figure 39: Activity of HMGS from female AC extracts. The reaction was performed by the DNTB-CoA assay. $1 \mathrm{mg}$ of protein was added in each of the three points; $24 \mathrm{~h} 20 \%$ SF, $96 \mathrm{~h} \mathrm{20 \%}$ SF and $96 \mathrm{~h} \mathrm{0 \%}$ SF females after adult eclosion. Each point ( \pm SEM) represents the mean of 3 biological replicates of extracts from $5 \mathrm{AC}$ from female mosquitoes. Significant differences $(\mathrm{p}<0.05)$ were determined with one way ANOVA followed by Tukey's test. 


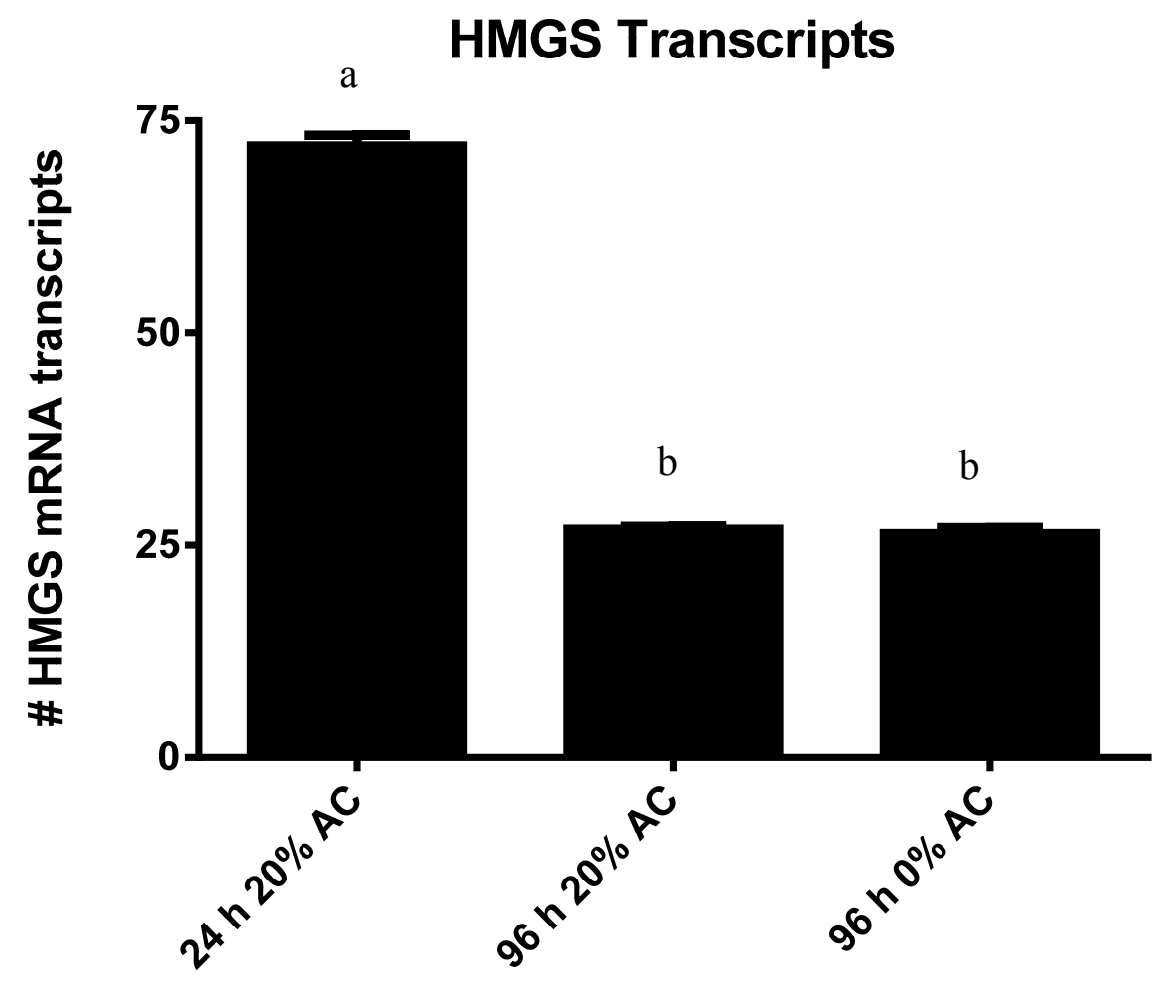

Figure 40: Expression of HMGS mRNAs from female abdominal carcass (AC) extracts. Expression of HMGS AC mRNAs of $24 \mathrm{~h} \mathrm{20 \%}$ SF, $96 \mathrm{~h} \mathrm{20 \%} \mathrm{SF}$ and $96 \mathrm{~h} \mathrm{0 \%} \mathrm{SF}$ females after adult eclosion. HMGS mRNAs are expressed as copy number of HMGS mRNA/10,000 copies of rpl32 mRNA. Each RT-PCR data point is average of three independent biological replicates of 5 AC. Significant differences $(p<0.05)$ were determined with one way ANOVA followed by Tukey's test. 


\subsection{References}

1. Aaron JA, Christianson DW (2010) Trinuclear metal clusters in catalysis by terpenoid synthases. Pure Appl Chem 82:1585-1597.

2. Andreassi JL, Vetting MW, Bilder PW, Roderick SL and Leyh TS (2009) Structure of the ternary complex of phosphomevalonate kinase: the enzyme and its family. Biochemistry 48(27):6461-8.

3. Belles X, Martin D, Piulachs MD (2005) The mevalonate pathway and the synthesis of juvenile hormone in insects. Ann Rev Entomol 50:181-199.

4. Braverman N, Steel G, Obie C, Moser A, Moser H, Gould SJ and Valle D (1997) Human PEX7 encodes the peroxisomal PTS2 receptor and is responsible for rhizomelic chondrodysplasia punctata. Nat Genet 15(4): 369-376.

5. Buesa C, Martınez-Gonzalez J, Casals N, Haro D, Piulachs MD, et al. (1994) Blattella germanica has two HMG-CoA synthase genes. Both are regulated in the ovary during the gonadotrophic cycle. J Biol Chem 269:11707-13.

6. Cabano J, Buesa C, Hegardt FG, Marrero PF (1997) Catalytic properties of recombinant 3-hydroxy-3-methylglutaryl coenzyme A synthase-1 from Blattella germanica. Insect Biochem Mol Biol 27:499-505.

7. Chang Q, Yan XX, Gu SY, Liu JF and Liang DC (2008) Crystal structures of human phosphomevalonate kinase at 1.8 A resolution. Proteins 73:254-258.

8. Cheek S, Zhang H, Grishin NV (2002) Sequence and structure classification of kinase. J Mol Biol 320:855-881.

9. Chen A, Kroon PA, Pouter CD (1994) Isoprenyl diphosphate synthases: Protein sequence comparisons, a phylogentic tree, and predictions of secondary structure. Prot Sci 3:600-607.

10. Clinkenbeard KD, Sugiyama T, Lane MD. 1975. Cytosolic 3-hydroxy-3methylglutaryl-CoA synthase from chicken liver. Methods Enzymol 35: 160-167.

11. Doun SS, Burgner JW, Briggs SD, Rodwell VW (2005) Enterococcus faecalis phosphomevalonate kinase. Prot Sci 14:1134-1139.

12. Eyzaguirre J, Valdebenito D, Cardemil E (2006) Pig liver phosphomevalonate kinase: Kinetic mechanism. Arc Biochem Biophy 454: 189-196.

13. Frick S, Nagelb R, Schmidtb A, Bodemanna RR, Rahfelda P, Paulsa G, Brandtc W, Gershenzonb J, Bolanda W and Bursea A (2013) Metal ions control product 
specificity of isoprenyl diphosphate synthases in the insect terpenoid pathway. Proc Natl Acad Sci USA 110(11):4194-9.

14. Fujihashi M, Zhang YW, Higuchi Y, Li XY, Koyama T and Miki K (2001) Crystal structure of cis-prenyl chain elongating enzyme, undecaprenyl diphosphate synthase. Proc Natl Acad Sci USA 98: 4337-4342.

15. Gabelli SB, McLellan JS, Montalvetti A, Oldfield E, Docampo R, Amzel LM (2006) Structure and mechanism of the farnesyl diphosphate synthase from Trypanosoma cruzi: implications for drug design. Proteins 62:80-88.

16. Garcia DE, Keasling JD (2014) Kinetics of phosphomevalonate kinase from Saccharomyces cerevisiae. Plos One 9(1):e87112. doi: 10.1371/journal.pone.0087112.

17. Goldstein JL, Brown MS (1990) Regulation of the mevalonate pathway. Nature $343: 425-30$.

18. Hellig H and Popjak G (1961a) Biosynthesis of cholesterol XIII Phosphomevalonic kinase from liver." J Lipid Res 2: 235-243.

19. Hellig $\mathrm{H}$ and Popjak $\mathrm{G}$ (1961b) Phosphomevalonic kinase and 5pyrophosphomevalonic anhydrodecarboxylase from pig liver. Biochemical J 880:4748.

20. Herdendorf TJ and Miziorko HM (2006) Phosphomevalonate kinase: functional investigation of the recombinant human enzyme. Biochemistry 45:3235-3242.

21. Herdendorf TJ and Miziorko HM (2007) Functional evaluation of conserved basic residues in human phosphomevalonate kinase. Biochemistry 46:11780-11788.

22. Hosfield DJ, Zhang Y, Dougan DR, Broun A, Tari LW, Swanson RV and Finn J (2004) Structural basis for bisphosphonate-mediated inhibition of isoprenoid biosynthesis. J Biol Chem 279:8526-8529.

23. Miziorko HM, Lane MD (1977) 3-Hydroxy-3-methylgutaryl-CoA synthase. Participation of acetyl-S-enzyme and enzyme-Shydroxymethylgutaryl-SCoA intermediates in the reaction. J Biol Chem 252:1414-1420.

24. Nagegowsa DA, Bach TJ, Chye ML (2004) Brassica juncea 3-hydroxy-3methylglutaryl (HMG)-CoA synthase 1: expression and characterization of recombinant wild-type and mutant enzymes Biochem J 383:517-527.

25. Ogura K, Koyama T (1998) Enzymatic aspects of isoprenoid chain elongation. Chem Rev 98:1263-1276. 
26. Ogura K, Toyama T, Sagami H (1997) Polyprenyl diphosphate synthases. In: Bittman R, editor. Subcellular biochemistry. New York: Plenum Press. p 57-88.

27. Olson AL, Yao H, Herdendorf TJ, Miziorko HM, Hannongbua S, Saparpakorn P Cai S, Sem DS (2009) Substrate induced structural and dynamics changes in human phosphomevalonate kinase and implications for mechanism. Proteins 75:127-138.

28. Pilloff D, Dabovic K, Romanowski MJ, Bonanno JB, Doherty M, et al. (2003) The kinetic mechanism of phosphomevalonate kinase. J Biol Chem 278:4510-4515.

29. Pojer F, Ferrer JL, Richard SB, Nagegowda DA, Chye ML, Bach TJ, Noel JP (2006) Structural basis for the design of potent and species-specific inhibitors of 3-hydroxy3-methylglutaryl CoA synthases. Proc Natl Acad Sci USA 103:11491-11496.

30. Poulter CD (2006) Farnesyl diphosphate synthase. A paradigm for understanding structure and function relationships in E-polyprenyl diphosphate synthases. Phytochem Rev 5:17-26.

31. Poulter CD and Rilling HC (1978) The prenyl transfer reaction. Enzymatic and mechanistic studies of the 1c-4 coupling reaction in the terpene biosynthetic pathway. Acc Chem Res 11:307-313.

32. Rivera-Perez C, Nouzova M, Lamboglia I, Noriega FG (2014) Metabolic analysis reveals changes in the mevalonate and juvenile hormone synthesis pathways linked to the mosquito reproductive physiology. Insect Biochem Mol Biol 51:1-9.

33. Romanowski MJ, Bonanno JB and Burley SK (2002) Crystal structure of the Streptococcus pneumoniae phosphomevalonate kinase, a member of the GHMP kinase superfamily. Proteins 47:568-571.

34. Rondeau J-M, Bitsch F, Bourgier E, Geiser M, Hemmig R, Kroemer M, Lehmann S, Ramage P, Rieffel S, Strauss A, Green JR, Jahnke W (2006) Structural basis for the exceptional in vivo efficacy of bisphosphonate drugs. Chem Med Chem 1:267-273.

35. Sato M, Sato K, Nishikawa SI, Hirata A, Kato JI, Nakano A (1999) The yeast RER2 gene, identified by endoplasmic reticulum protein localization mutations, encodes cisprenyltransferase, a key enzyme in dolichol synthesis. Mol Cell Biol 19:471-483.

36. Smit A and Mushegian A (2000) Biosynthesis of isoprenoids via mevalonate in Archaea: the lost pathway, Genome Res 10:1468-1484. 
37. Sutherlin A, Hedl M, Sanchez-Neri B, Burgner JW, II, Stauffacher CV, Rodwell VW (2002) Enterococcus faecalis 3-hydroxy-3-methylglutaryl coenzyme A synthase, an enzyme of isopentenyl diphosphate biosynthesis. J Bacteriol 184:4065-4070.

38. Taban HA, Tittiger C, Blomquist GJ and William H (2009). WelchIsolation and characterization of farnesyl diphosphate synthase from the cotton boll weevil, Anthonomus grandis. Arch Insect Biochem Physiol 71:88-104.

39. Tarshis LC, Proteau PJ, Kellogg BA, Sacchettini JC and Poulter CD (1996) Regulation of product chain length by isoprenyl diphosphate synthases. Proc Natl Acad Sci USA 93:15018-15023.

40. Tarshis LC, Yan M, Poulter CD and Sacchettini JC (1994) Crystal structure of recombinant farnesyl diphosphate synthase at $2.6 \mathrm{~A}{ }^{\circ}$. Biochemistry 33: 1087110877.

41. Tittiger C, O'Keeffe C, Bengoa CS, Barkawi LS, Seybold SJ, Blomquist GJ (2000) Isolation and endocrine regulation of an HMG-CoA synthase cDNA from the male Jeffrey pine beetle, Dendroctonus jeffreyi (Coleoptera: Scolytidae). Insect Biochem Mol Biol 30:1203-11.

42. Tomoda H, Kumagai H, Takahashi Y, Tanaka Y, Iwai Y, Omura S (1988) F-244 (1233A), a specific inhibitor of 3-hydroxy-3-methylglutaryl coenzyme A synthase: taxonomy of producing strain, fermentation, isolation and biological properties. $\mathrm{J}$ Antibiot 41:247-249.

43. VanNice JC, Skaff DA, Wyckoff GJ and Miziorko HM (2013) Expression in Haloferax volcanii of 3-hydroxy-3-methylglutaryl coenzyme A synthase facilitates isolation and characterization of the active form of a key enzyme required for polyisoprenoid cell membrane biosynthesis in halophilic archaea. J Bacteriol 195:3854-3862.

44. Walker JE, Saraste M, Runswick MJ and Gay NJ (1982) Distantly related sequences in the a- and $\beta$-subunits of ATP synthase, myosin, kinases and other ATP-requiring enzymes and a common nucleotide binding fold. EMBO J 1:945-951.

45. Wanders RJA and Romeijn GJ (1998). Differential deficiency of mevalonate kinase and phosphomevalonate kinase in patients with distinct defects in peroxisome biogenesis: evidence for a major role of peroxisomes in cholesterol biosynthesis. Bichem Biophys Res Commun 247: 663-667. 


\section{Chapter 5: Conclusions and future directions}

\subsection{Conclusions}

This dissertation is a comprehensive study of five Aedes aegypti CA enzymes, HMGS, MK, PMK, FPPS, and FPPase involved in JH synthesis. The study mainly focused on the expression and characterization of recombinant protein, the analysis of their kinetics and inhibition constants, as well as the understanding the importance of these enzymes in the control of $\mathrm{JH}$ biosynthesis rates.

In chapter 2 FPP phosphatase responsible for the transformation of FPP into FOL in the CA of A. aegypti was characterized at molecular and biochemical levels. Some of the observations from chapter 2 include:

1. Identification of AaFPPase- 1 and AaFPPase- 2 as members of the NagD family of the Class IIA C2 cap-containing haloalkanoic acid dehalogenase (HAD) super family that efficiently hydrolyzed FPP and GPP but not IPP.

2. Different FPPase activities in CA of sugar-fed as compared to blood-fed females.

3. Injection of dsRNAs resulted in a significant reduction of both AaFPPase-1 and AaFPPase-2 mRNAs, but only former resulted in a significant decrease of JH biosynthesis.

4. AaFPPase-1 appears to be the major enzyme involved in the catalysis of FPP into FOL in the CA of A. aegypti.

5. N-acetyl-S-geranylgeranyl-L-cysteine appears as a potent inhibitor of AaFPPase 1 and AaFPPase 2 in blocking JH synthesis in A. aegypti.

Chapter 3 describes the first characterization of a mevalonate kinase in insects. 
Some of the conclusions of the studies included are:

1. AaMK is a class I MK that has the typical structure and functional features of other members of the GHMP kinase family.

2. Recombinant $A a \mathrm{MK}$ is found to be inhibited by isoprenoids; with a pattern similar to those of other eukaryotic MKs.

3. Inhibition of the activity of MKs by isoprenoids is found to be competitive with respect to ATP and the eukaryotic enzymes are 1000-fold more sensitive to the inhibition by isoprenoids than bacterial and archaeal enzymes.

4. The MK activity in thoraces extracts of sugar-fed (SF) mosquitoes was also significantly reduced in the presence of FPP.

Chapter 4 reports the partial characterization of the activities of recombinant HMGS, PMK and FPPS from A. aegypti. Some of the conclusions of the studies included are:

1. HMGS transcripts do not change with the nutritional condition of the insects. However its activity was reduced when mosquitoes are starved for three days.

2. Hymeglusin is a specific inhibitor of HMGS that could be used to block the mevalonate pathway in A. aegypti.

3. AaPMK is a member of the nucleoside monophosphate family.

4. The present study is the first report on kinetics of AaPMK for both forward and backward reactions in insects. AaPMK catalytic activity is not inhibited by any of the downstream metabolites.

5. Recombinant AaFPPS possesses an interesting product regulation mechanism; it alters the chain length of its products depending on the cofactor present. 


\subsection{Future directions}

All the thirteen corpora allata enzymes involved in the pathway of juvenile hormone biosynthesis have been identified in the mosquito A. aegypti. With the inclusion of appropriate cofactors, CA extracts are capable of de novo synthesis of $\mathrm{JH}$ metabolites from precursors (Rivera-Perez et al., 2013, 2014; Nyati et al., 2013); these in vitro assay have been used to study the endogenous activities of eight of these enzymes, HMGS, MK, PMK, FPPS, FPPase, FOLD, FALD and JHAMT. These eight enzymes along with IPPI have been expressed as recombinant proteins and their activities were characterized in vitro (Mayoral et al., 2009a, 2009b; Diaz et al., 2012; Rivera-Perez et al., 2013; Nyati et al., 2013). Only four enzymes remain to be characterized in mosquitoes, acetoacetylCoA thiolase, HMGR, MDD and epoxidase.

The enzymes of the JH-branch have been considered as more suitable for insecticides target of $\mathrm{JH}$ biosynthesis; given that inhibitors targeting them are less likely to affect non-insect organisms. Inhibitors directed at the MVP enzymes may lack insectspecificity; however, in some groups of insects, MVP enzymes may display unique features that will permit the development of target-specific inhibitors (Cousson et al., 2013). There are examples of such effective inhibitors; 6-fluoromevalonate 5-diphosphate fluoromevalonolactone (FMev) completely inhibits the activity of lepidopteran MDD (Quistad et al., 1981; Baker et al., 1986; Cusson et al., 2013). Inhibitors of FPPSs, such as lipophilic bisphosphonates, have been designed as drugs and herbicides (Oldfield, 2010; Cromartie, 1991). Lipophilic bisphosphonates effectively inhibit protein prenylation and invasiveness in tumor cells and has been used as anticancer agents (Zhang et al., 2009; Oldfield, 2010). The completion of the functional analyses of all the 
enzymes involved in the biosynthesis of $\mathrm{JH}$ in A. aegypti will permit the search for specific inhibitors that target some of these enzymes and disrupt the mosquito reproductive physiology.

Recombinant enzymes have been used in building of wide range of natural and synthetic isoprenoids to be used as medicines, cosmetics, flavors, fragrances and biofuels (Hale et al., 2007; Lacaze et al., 2011; Peralta-Yahya et al., 2011; Zhang et al., 2011). Recent attempts include the production of isoprenoid-based biofuels that might help to overcome rising petroleum costs, trade imbalances and environmental concerns (PeraltaYahya et al., 2011; Zhang et al., 2011). Artemisinin, an antimalarial drug has been enzymatically synthesized from acetyl-CoA using genetic engineering at very low costs (Hale et al., 2007; Lacaze et al., 2011). Similar approaches could be applied for the development of a multienzyme JH pathway bioreactor. The enzymes of the JH pathway may be segregated in a module, immobilized on a membrane, or attached to a support for the development of the JH bioreactor. This could result in significant enhancements in yield, purity, production time and cost when compared to traditional chemical synthetic methods for the synthesis of JHs, pheromones, defensive secretions and many other isoprenoid-derived compounds. Additionally, linking the bioreactor with the HPLCfluorescent detection assay could help in the study of metabolites flux and changes in pool sizes, helping to model $\mathrm{JH}$ regulation, as well as the design of a multi-enzyme inhibitor. 


\subsection{References}

1. Cromartie TH, Fisher KJ, Grossman JN (1991) Discovery of a novel site of action for herbicidal bisphosphonates. Pestic Biochem Physiol 63:114-126.

2. Cusson M, Sen SE, Shinoda T (2013) Juvenile Hormone Biosynthetic Enzymes as Targets for Insecticide Discovery. In Advanced Technologies for Managing Insect Pests (Ishayya I, Palli SR, Horowitz AR eds), Springer pp. 31-55.

3. Diaz M, Mayoral JM, Priestap H, Nouzova M, Rivera-Perez C, Noriega FG (2012) Characterization of an isopentenyl diphosphate isomerase involved in the juvenile hormone pathway in Aedes aegypti. Insect Biochem Molec Biol 42:751-757.

4. Mayoral JG, Nouzova M, Navare A, Noriega FG, (2009a) NADP ${ }^{+}$dependent farnesol dehydrogenase, a corpora allata enzyme involved in juvenile hormone synthesis. Proc Natl Acad Sci USA 106:21091-21096.

5. Mayoral JG, Nouzova M, Yoshiyama M, Shinoda T, Hernandez-Martinez S, Dolghih E, Turjanski AG, Roitberg AE, Priestap H, Perez M, Mackenzie L, Li Y, Noriega FG, (2009b) Molecular and functional characterization of a juvenile hormone acid methyltransferase expressed in the corpora allata of mosquitoes. Insect Biochem Mol Biol 39:31-37.

6. Nyati P, Nouzova M, Rivera-Perez C, Clifton ME, Mayoral JG Noriega FG (2013) Farnesyl phosphatase, a corpora allata enzyme involved in juvenile hormone synthesis in Aedes aegypti. PLoS ONE 8(8): e71967. doi:10.1371/journal.pone.0071967.

7. Nyati P, Rivera-Perez C, Noriega FG Negative feedback by isoprenoids upon mevalonate kinase activity might regulate juvenile hormone synthesis in Aedes aegypti. In prep

8. Hale V, Keasling JD, Renninger N, Diagana TT (2007) Microbially derived artemisinin: a biotechnology solution to the global problem of access to affordable antimalarial drugs. Am J Trop Med Hyg 77:198-202.

9. Hucka, M, Finney A, Sauro HM, Bolouri H, Doyle JC, Kitano H et al., (2003) The systems biology markup language (SBML): a medium for representation and exchange of biochemical network models. Bioinformatics 19:524-531. 
10. Lacaze C, Kauss T, Kiechel JR, Caminiti A, Fawaz F, et al. (2011) The initial pharmaceutical development of an artesunate/amodiaquine oral formulation for the treatment of malaria: a public-private partnership. Malar J 10:142.

11. Oldfield E (2010) Targeting isoprenoid biosynthesis for drug discovery: bench to beside. Acc Chem Res 43:1216-1226.

12. Oldfield E, Lin FY (2012) Terpene biosynthesis: modularity rules. Angew Chem Int Ed Engl 51:1124-1137.

13. Peralta-Yahya PP, Ouellet M, Chan R, Mukhopadhyay A, Keasling JD, et al. (2011) Identification and microbial production of a terpene-based advanced biofuel. Nat Commun 2:483.

14. Quistad GB, Cerf DC, Schooley DA, Staal GB (1981) Fluoromevalonate acts as an inhibitor of juvenile hormone biosynthesis. Nature 289:176-177.

15. Rivera-Perez C, Nouzova M, Clifton ME, Martin Garcia E, LeBlanc E, Noriega FG (2013) Aldehyde dehydrogenase 3 converts farnesal into farnesoic acid in the corpora allata of mosquitoes. Insect Biochem Mol Biol 43:675-682.

16. Rivera-Perez C, Nouzova M, Lamboglia I, Noriega FG (2014) Metabolic analysis reveals changes in the mevalonate and juvenile hormone synthesis pathways linked to the mosquito reproductive physiology. Insect Biochem Mol Biol 51:1-9.

17. Rivera-Perez C, Nyati P, Noriega FG Characterization of a farnesyl pyrophosphate synthase involved in juvenile hormone biosynthesis in Aedes aegypti. In prep

18. Zhang F, Rodriguez S, Keasling JD (2011) Metabolic engineering of microbial pathways for advanced biofuels production. Curr Opin Biotechnol 22:775-783.

19. Zhang Y, Cao R, Yin F (2009) Lipophilic bisphosphonates as dual farnesyl/geranylgeranyl diphosphate synthase inhibitors: an X-ray and NMR investigation. J Am Chem Soc 131: 5153-5162. 
VITA

\section{PRATIK NYATI}

Born, Indore, India

2004-2007

B.S., Biotechnology

Holkar Science College

Indore, India

2007-2009

M.S., Biotechnology

Devi Ahilya Vishwavidyalaya

Indore, India

2009-2014

Doctoral Candidate

Florida International University

Miami, Florida

Teaching Assistant

Florida International University

Miami, Florida

\section{PUBLICATIONS AND PRESENTATIONS}

Nyati P, Nouzova M, Mayoral JG, Noriega FG. Identification and characterization of a Farnesyl pyrophosphatase involved in juvenile hormone synthesis in the Aedes aegypti mosquito. $13^{\text {th }}$ annual FIU Biomedical and Comparative Immunology Symposium. March $3^{\text {rd }}-4^{\text {th }} 2011$ Florida International University, Miami, Florida.

Nyati P, Nouzova M, Rivera-Perez C, Clifton ME, Mayoral JG, Noriega FG (2013) Farnesyl Phosphatase, a Corpora allata Enzyme Involved in Juvenile Hormone Biosynthesis in Aedes aegypti. PLoS ONE 8(8): e71967. doi:10.1371/journal.pone.0071967.

Nyati P, Rivera-Perez C, Noriega FG. Characterization of mosquito juvenile hormone biosynthetic enzymes. $13^{\text {th }}$ annual Meeting of Entomological Society of America, November $11^{\text {th }}-13^{\text {th }} 2013$, Austin, Texas.

Nyati P, Rivera-Perez C, Noriega FG. Negative feedbacks by isoprenoid intermediates on mevalonate kinase activity might regulate juvenile hormone synthesis in Aedes aegypti. $16^{\text {th }}$ annual FIU Biology Research Symposium. February $1^{\text {st }} 2014$ Florida International University, Miami, Florida. 
Nyati P, Rivera-Perez C, Noriega FG Negative feedbacks by isoprenoid intermediates on mevalonate kinase activity might regulate juvenile hormone synthesis in Aedes aegypti. In preparation

Rivera-Perez C, Nyati P, Noriega FG A corpora allata prenyltransferase in mosquito dispaying a metal ion substrate specificity. In preparation 